\title{
DEVELOPMENT AND IMPROVEMENT OF ARTIFICIAL DIETS FOR LARVAE OF DIABROTICA SPECIES USING MULTIDIMENSIONAL DESIGN SPACE TECHNIQUES
}

\author{
A Dissertation \\ presented to
}

the Faculty of the Graduate School

at the University of Missouri-Columbia

In Partial Fulfillment

of the Requirements for the Degree

Doctor of Philosophy

by

MAN P. HUYNH

Dr. Thomas A. Coudron, Dissertation Supervisor

APRIL 2018 
The undersigned, appointed by the dean of the Graduate School, have examined the dissertation entitled

\section{DEVELOPMENT AND IMPROVEMENT OF ARTIFICIAL DIETS FOR LARVAE OF DIABROTICA SPECIES USING MULTIDIMENSIONAL DESIGN SPACE TECHNIQUES}

Presented by Man P. Huynh

A candidate for the degree of

Doctor of Philosophy

and hereby certify that, in their opinion, it is worthy of acceptance.

Dr. Deborah L. Finke

Dr. Thomas A. Coudron

Dr. Bruce E. Hibbard

Dr. Kevin L. Fritsche 
This dissertation is dedicated to my wife, Van Ho, and my sons, Tri Huynh and Huy Huynh for their love, support, and sacrifices throughout my graduate studies.

I am truly thankful for having my beloved family in my life. 


\section{ACKNOWLEDGEMENTS}

I would like to thank my advisors Thomas Coudron and Deborah Finke for their invaluable insight, guidance and support over the past four years. I would like to thank Bruce Hibbard and Kevin Fritsche for his helpful advice on my research. I would like to also thank Kent Shelby, Stephen Lapointe, Randall Niedz, Lisa Meihls, Elisa Bernklau, Louis Bjostad, Wade French, Art Schaafsma, and Andrea Hitchon for their collaboration.

My research would not have been possible if it weren't for the tireless efforts of everyone in our corn rootworm group in the USDA laboratories in Columbia, Missouri. I would especially like to thank Julie Barry, James Smith, Michelle Gregory, Dalton Ludwick, Adriano Pereira, Emily Harrington, Zixiao Zhao, and Brock Andreasen for the many of things they have helped me with my experiments. I would also like to thank Alexander Brittani, Joseph Larose, Kathryn Ingerslew, Jessica Kansman for their help with my graduate studies.

I thank the University of Missouri Division of Plant Sciences and the USDA Biological Control of Insects Research Laboratory for making my graduate studies possible. I would like to thank entire Entomology faculty and my fellow graduate students that have been very supportive. I thank the Can Tho University Department of Plant Protection for the support on my graduate studies.

Finally, I would like to thank to my family, especially my parents, Danh Huynh and Dao Tran and my parents-in-law, Han Tran and Nghia Ho who have given me their limitless encouragement and support. 


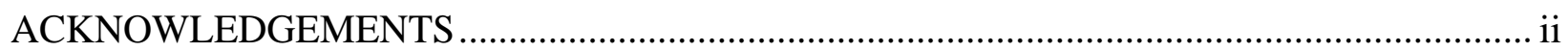

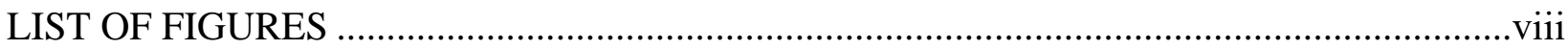

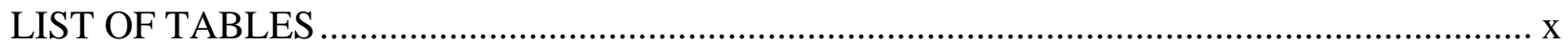

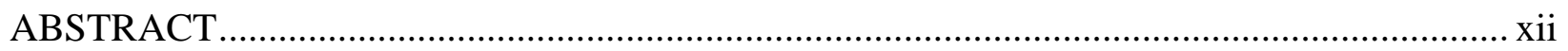

CHAPTER I: INTRODUCTION AND LITERATURE REVIEW ..............................................

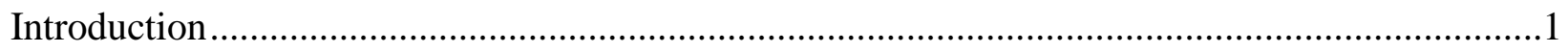

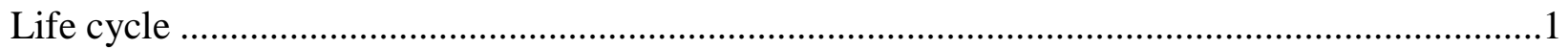

The development of resistance of corn rootworms .......................................................................

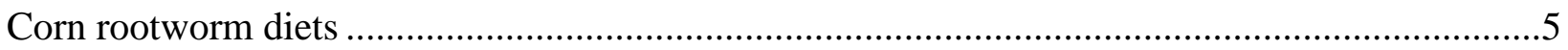

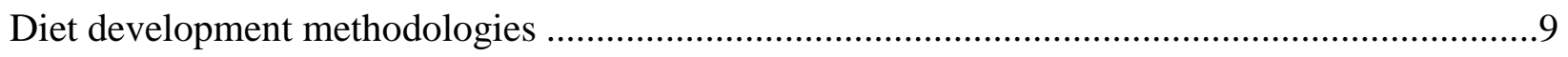

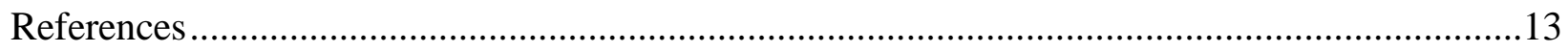

CHAPTER II: DIET IMPROVEMENT FOR WESTERN CORN ROOTWORM LARVAE .....21

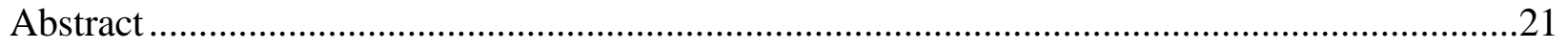

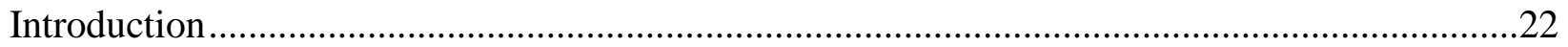

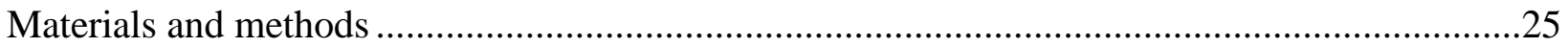

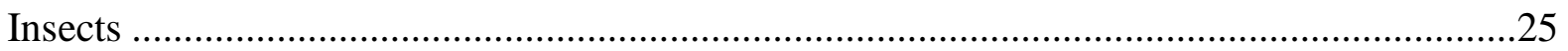

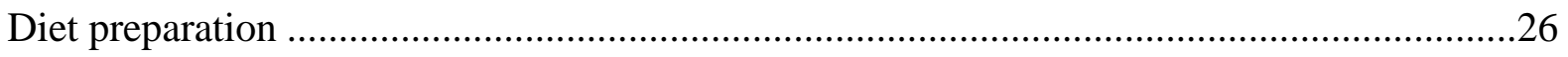

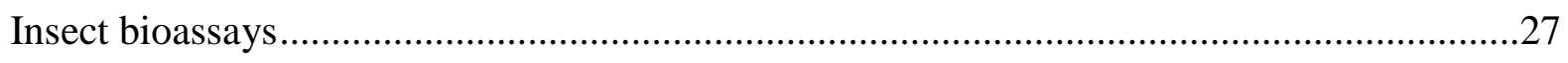

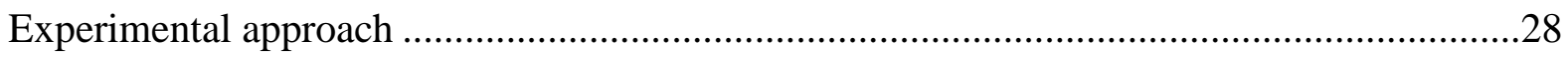

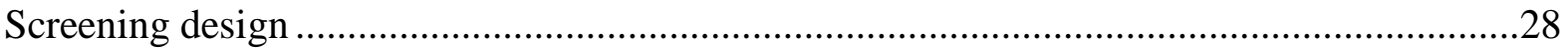

4-component mixture design..............................................................................................29

2-component mixture design for lipid components …………………....................................30

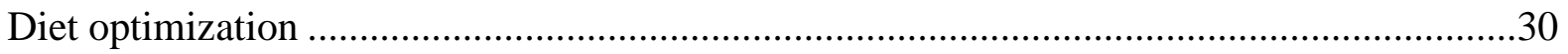


Model evaluation

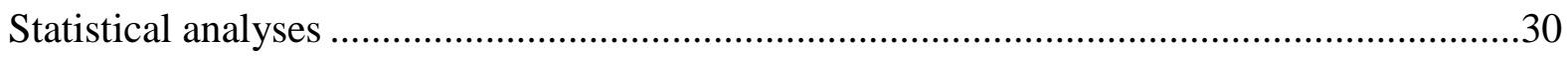

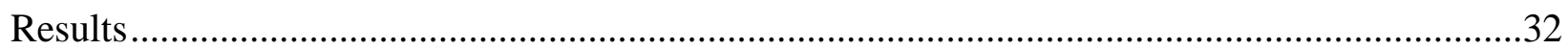

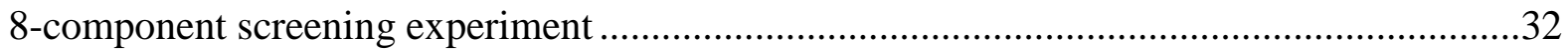

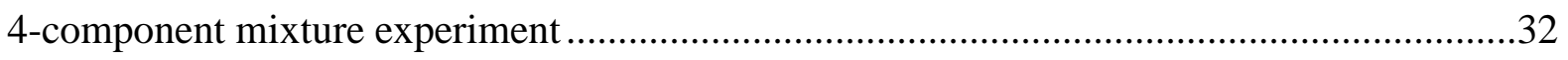

2-component lipid mixture experiment .....................................................................35

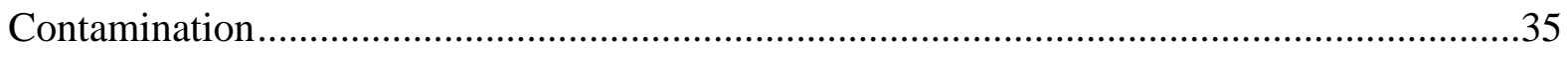

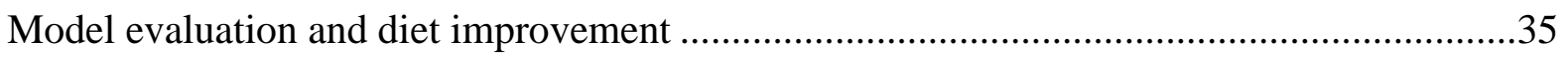

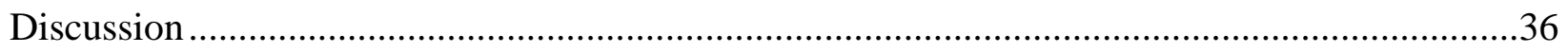

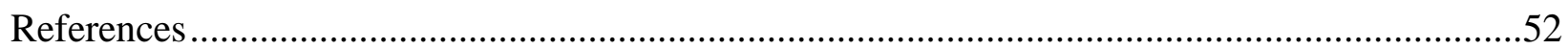

CHAPTER III: ISOLATION AND CHARACTERIZATION OF CORN ROOT FACTORS FOR IMPROVED GROWTH OF WESTERN CORN ROOTWORM LARVAE REARED ON

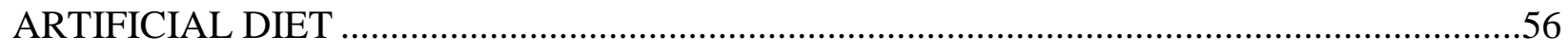

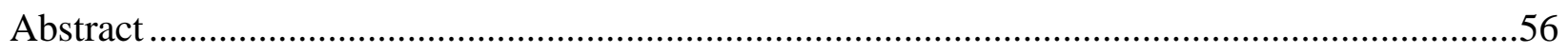

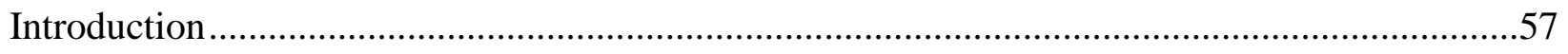

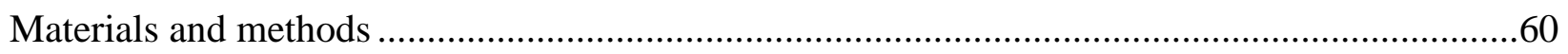

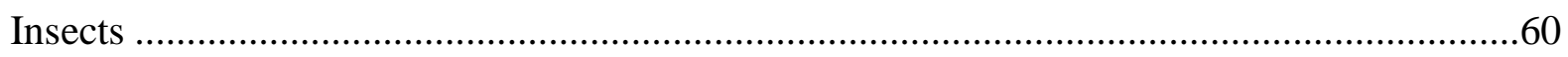

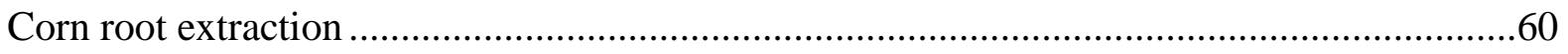

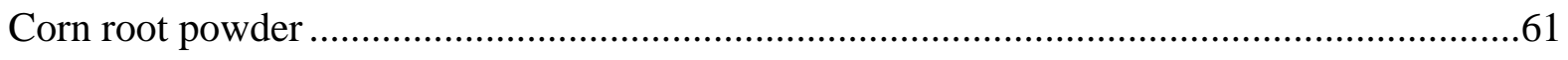

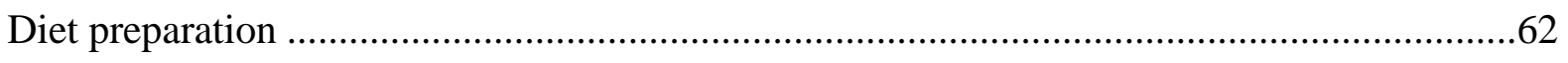

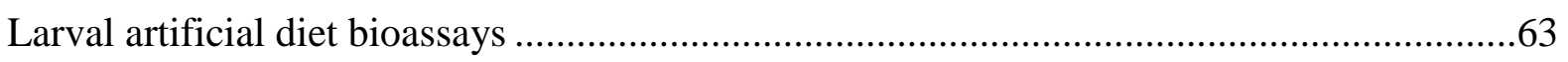

Diet bioassays with corn root extract.....................................................................64

Diet bioassays with corn root powder as a major nutritional source ..................................64

Diet bioassays with corn root powder from different seeds and suppliers .........................65

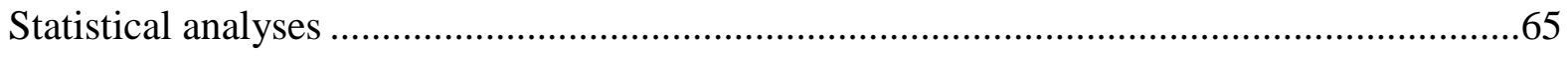

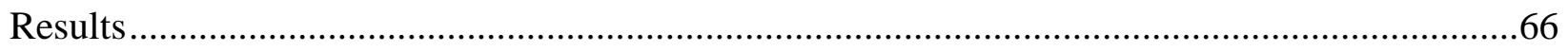




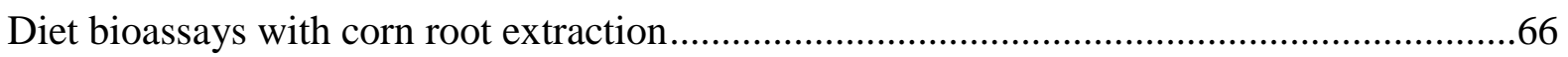

Diet bioassays with corn root powder as the major nutritional source ....................................66

Diet bioassays with corn root powder form different seeds and suppliers ..............................67

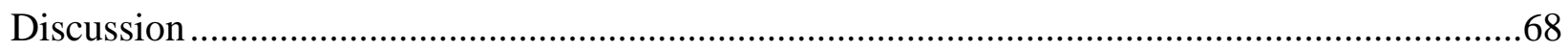

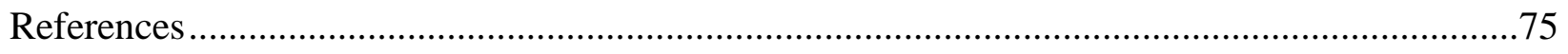

CHAPTER IV: MIXTURE DESIGN AND RESPONSE SURFACE MODELING TO

DEVELOP AN IMPROVED AND ACCESSIBLE DIET FOR WESTERN CORN

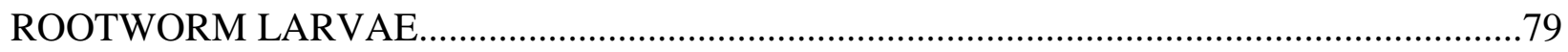

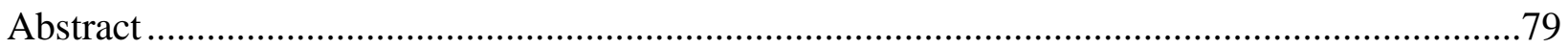

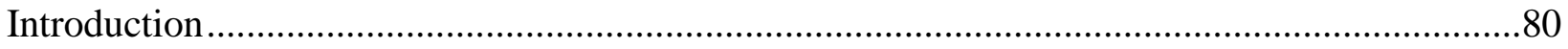

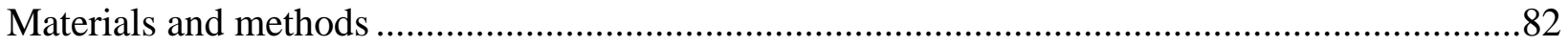

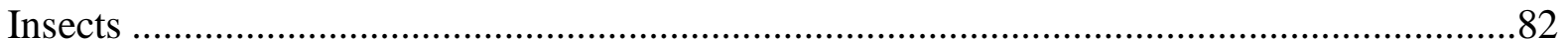

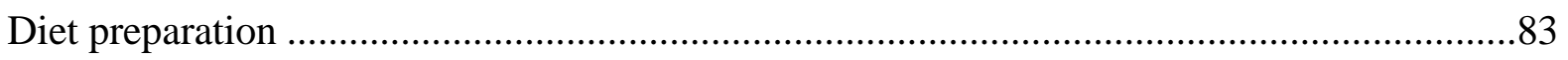

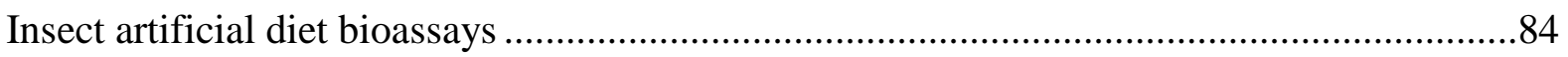

Eight-protein screening design for substitutes for corn root powder......................................85

Three-protein mixture-amount design for characterizing key protein sources .........................85

Egg powder experiment to identify optimum formulation ..................................................8

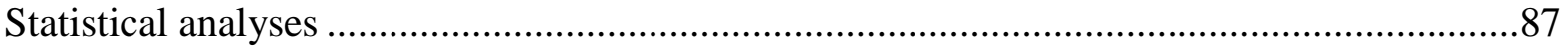

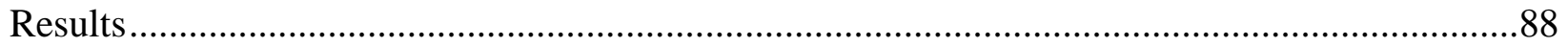

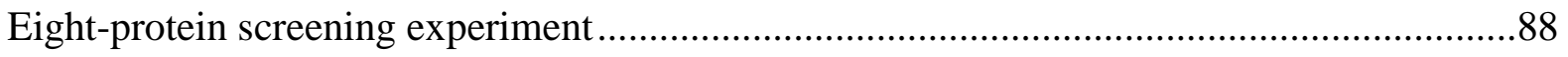

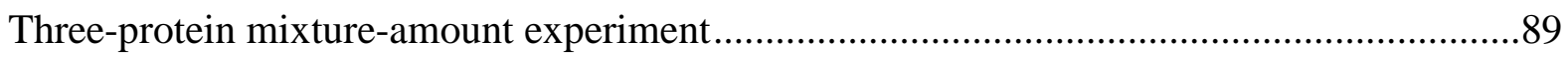

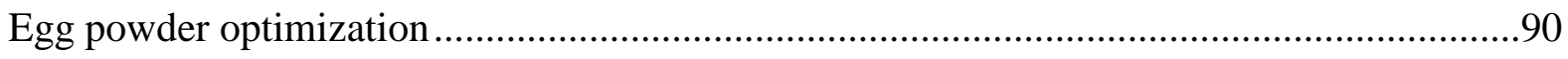

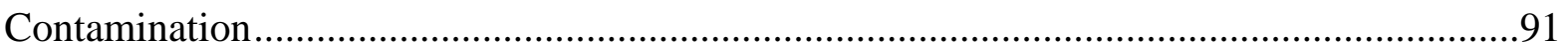

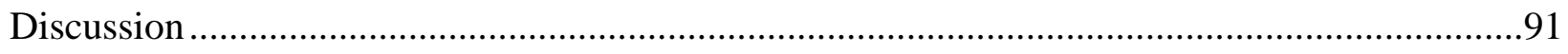

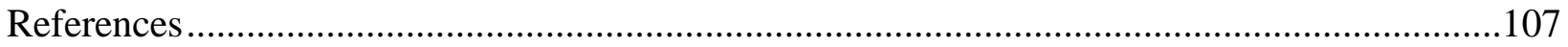


Abstract

Introduction

Materials and methods

Insects

Diet preparation

Insect diet bioassays.

Experimental approach

Comparison of five rootworm diets

8-component mixture design for basic nutrition profile

8-protein screening design

3-protein mixture design ......

Diet optimization .120

Model validation 120

Statistical analyses 120

Results

Comparison of five rootworm diets

8-component screening experiment for basic nutrition profile.

8-protein mixture experiment

3-protein mixture-amount experiment

Contamination

Diet optimization

Model validation and diet improvement

Discussion 


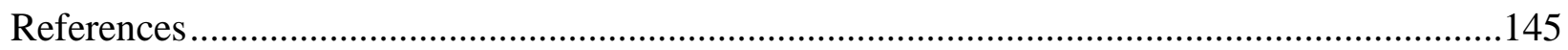

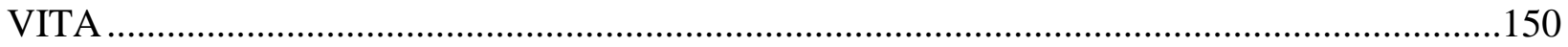




\section{LIST OF FIGURES}

Figure 1. Piepel trace plot of larval weight deviation from a reference blend diet.

Figure 2. Predicted 3-D surface response plots for WCR larvae reared on different diets. .48

Figure 3. Nonlinear blending effects in the 4-component mixture experiment.....

Figure 4. Piepel trace plots of weight, survival and molt deviation from a reference diet .50

Figure 5. Predicted survival plots for WCR larvae reared on diets that vary in lipid content.......51

Figure 6. Survival, dry weight, molt to $2^{\text {nd }}$ instar, incomplete molt to $2^{\text {nd }}$ instar, molt to $3^{\text {rd }}$ instar of western corn rootworm larvae reared on diets with different types of corn roots

Figure 7. Survival, and average dry weight of western corn rootworm larvae reared on diet formulations containing corn root powder as the major macronutrient component

Figure 8. Survival, average dry weight, molt to $2^{\text {nd }}$ instar, molt to $3^{\text {rd }}$ instar of western corn rootworm larvae reared on diet formulations differing in corn root powder sources

Figure 9. Trace plot of WCR larval responses deviation from a reference blend diet in 8-protein screening experiment.

Figure 10. Contour plots of larval responses for WCR reared on different diets from mixtureamount design of casein:egg powder:whey protein at 10 days post infestation.

Figure 11. Nonlinear blending effects of egg powder $\times$ whey protein on molting from the mixture-amount experiment

Figure 12. Contour plots of the interaction of egg powder, casein and concentration on WCR larvae reared on different diets from the mixture-amount design at 10 days post infestation.....103

Figure 13. Larval dry weight, survival, and percent successful completion of molt for western corn rootworm larvae reared on different diets 104 
Figure 14. Larval dry weight, survival, and percent successful completion of molt for western corn rootworm larvae reared on WCRMO-2, WCRMO-1, and a proprietary diet for 10 days ...105

Figure 15. Percent successful completion of molt for western corn rootworm larvae reared on WCRMO-2, WCRMO-1, and a proprietary diet for 10 days.

Figure 16. Percentage survival, average dry weight, percentage molting to $2^{\text {nd }}$ instar of northern corn rootworm larvae fed different diet formulations

Figure 17. Cox plot of larval responses to deviations from a reference blend diet in the proportions of ingredients in an 8-component mixture experiment.

Figure 18. Cox plot of larval responses to deviations from a reference blend diet in the proportions of ingredients in an 8-protein screening experiment

Figure 19. Contour plots of larval responses to a mixture-amount design of casein: egg powder: whey protein at 10 days post infestation

Figure 20. Nonlinear blending effects of casein $\times$ egg powder from the mixture-amount experiment.

Figure 21. Larval dry weight, percent successful completion of molt, and survival for northern corn rootworm larvae reared on NCRMO-1 and WCRMO-1 diets for 10 days. 143

Figure 22. Flow chart for diet development algorithm 144 


\section{LIST OF TABLES}

Table 1. Components that were proportionally varied or held constant in diets used to rear western corn rootworm larvae .40

Table 2. Diet blends of 8 components varied in a 7-dimensional, mixture screening design to rear western corn rootworm larvae

Table 3. Diet blends of 4 ingredients varied in a 3-dimensional mixture design to rear western corn rootworm larvae

Table 4. Diet blends of 2 lipid components varied in a 1-dimensional mixture design to rear western corn rootworm larvae

Table 5. $p$-values, regression coefficients and response surface model fitting diagnostic statistics for WCR larval responses to 4-component diet mixtures

Table 6. Diet formulation improvement for western corn rootworm development.

Table 7. Larval dry weight, survival, and percent successful completion of molt for western corn rootworm larvae reared on the WCRMO-1 and the Pleau et al. diets for 7 or 11 days .46

Table 8. Components in diets used to rear western corn rootworm larvae in corn root powder experiment

Table 9. Diet blends of 8 proteins (gram) varied in a mixture screening design to rear western corn rootworm larvae

Table 10. Components that were held constant in diets used to rear western corn rootworm larvae in the 8-protein mixture experiment

Table 11. Mixture-amount (concentration) design points to determine the optimum combination

of key protein ingredients (gram) .96

Table 12. $p$-values, regression coefficients and response surface model fitting diagnostic statistics for western corn rootworm larval responses to 8-component diet mixtures 
Table 13. $p$-values, regression coefficients and response surface model fitting diagnostic statistics for western corn rootworm larval responses to 3-protein mixture-amount experiment 98

Table 14. Artificial diets for western corn rootworm larvae

Table 15. Diet blends of 8 components varied in the mixture exploratory design to rear northern corn rootworm larvae 130

Table 16. Diet blends of 8-proteins (gram) varied in the mixture screening design to rear northern corn rootworm larvae

Table 17. Components that were held constant in diets used to rear western corn rootworm larvae in the 8-protein mixture experiment

Table 18. Mixture-amount design points to determine the optimum combination of key protein ingredients

Table 19. $p$-values, regression coefficients and response surface model fitting diagnostic statistics for NCR larval responses to 8-component diet mixtures

Table 20. $p$-values, regression coefficients and response surface model fitting diagnostic statistics for WCR larval responses to 8-protein diet mixtures

Table 21. $p$-values, regression coefficients and response surface model fitting diagnostic statistics for WCR larval responses to 3-protein mixture-amount experiment.

Table 22. An artificial diet for northern corn rootworm larvae 


\begin{abstract}
The western corn rootworm (Coleoptera: Chrysomelidae; Diabrotica virgifera virgifera LeConte) and the northern corn rootworm (Coleoptera: Chrysomelidae; Diabrotica barberi Smith \& Lawrence) are highly adaptive insect pests and have developed resistance to most possible management tactics in some regions. Transgenic maize hybrids, the latest control tools, which express insecticidal crystalline toxins from Bacillus thuringiensis (Bt) Berliner, have also faltered due to physiological adaptation of western corn rootworm populations. The United States Environmental Protection Agency has mandated insect resistance management plans for corn rootworms that have been directed toward monitoring the development of resistance to each of the Bt toxins. Toxicity bioassays using artificial diet have proven to be valuable for monitoring resistance to Bt toxins in corn rootworm populations. Currently, several proprietary diet formulations for western corn rootworm larvae that have been developed by each of the maize seed companies are used by industry and public researchers in toxicity bioassays to detect decreases in susceptibility of western corn rootworm populations to Bt proteins. No artificial diet has been developed specifically for northern corn rootworm larvae. Since differences in diet formulations can lead to different results in diet toxicity assays, it is impossible to make diet comparisons between toxicity assays using different artificial diets from individual companies and it may not be possible to determine an accurate picture of the test population's phenotype as it relates to Bt resistance. We report new diet formulations that support improved weight gain, larval development, and survival compared with current diets used in western corn rootworm and northern corn rootworm bioassays. The new formulations were created by using response surface modeling coupled with n-dimensional mixture designs to identify and optimize key ingredients based on integrated evaluation of several life history
\end{abstract}


parameters (i.e., weight, molting, survival) while limiting contamination. The new rootworm formulations supported approximately 97\% larval survival and molting and increased larval weight gain after 10 days of feedings compared to other diets used in western corn rootworm and northern corn rootworm bioassays rearing. These new formulations provide a standardized growth medium for western corn rootworm and northern corn rootworm larvae that will facilitate toxicity test comparisons conducted by different working groups and meets all regulatory requirements.

We developed an improved diet formulation (WCRMO-1) for western corn rootworm (Chapter 2), which was the optimization of diet ingredients in the only published diet for western corn rootworm larvae. This formulation was also compatible for use with all current Bt proteins targeting western corn rootworm larvae. However, this formulation contains corn root powder, which is not available for purchase, limiting the practical use of the diets. We demonstrated that essential growth factors in corn roots that assist in western corn rootworm growth can be extracted suggesting substituting corn root powder with compounds identified from the extract may be possible (Chapter 3). An improved and accessible diet for western corn rootworm (WCRMO-2) that is comparable with all current diets for western corn rootworm larvae and without corn root powder was created by exploring and characterizing protein ingredients from plant, animal and yeast products (Chapter 4). Lastly, we developed the first artificial diet (NCRMO-1) for northern corn rootworm (Chapter 5) comprised of available ingredients that supports performance of northern corn rootworm larvae better than that of publicly available rootworm formulations. 


\section{CHAPTER I: INTRODUCTION AND LITERATURE REVIEW}

\section{Introduction}

Diabrotica Chevrolat (Coleoptera: Chrysomelidae: Galerucinae) is a largely Neotropical genus (Wilcox 1972) that includes some of the most destructive insect pests (Metcalf 1986). In North America, western corn rootworm (WCR), Diabrotica virgifera virgifera LeConte, and northern corn rootworm, Diabrotica barberi Smith \& Lawrence (NCR), are the most serious pests of maize (Zea mays L.) (Branson and Krysan 1981, Krysan 1986, Gray et al. 2009). The economic impact stemming from yield loss and control costs associated with these two species has been estimated between $\$ 1-2$ billion annually in the United States (Mitchell 2011). Western corn rootworm became establishment on maize in Europe in 1992 (Bača 1994) and now this pest has invaded 29 European countries (CABI 2017). In Europe, the damage costs caused by western corn rootworm have been estimated to be $€ 472$ million annually (Wesseler and Fall 2010).

\section{Life cycle}

Both western corn rootworm and northern corn rootworm have a univoltine life cycle with one generation per year, and overwinter as eggs (Ball 1957, Branson and Krysan 1981). The eggs are primarily laid in the soil in an obligate diapause (Krysan and Branson 1977) from late July through September. The eggs remain in the soil until hatching the following late May and early June (Levine and Oloumi-Sadeghi 1991). Larvae feed on corn roots and go through three larval instars below ground. Pupation occurs in the soil (Levine and Oloumi-Sadeghi 1991) and 
adults emerge from late June and early July (Murphy et al. 2010) to late August (Hughson and Spencer 2015).

Western corn rootworm develops successfully from hatch to adult stage from $15^{\circ} \mathrm{C}$ to $31.5^{\circ} \mathrm{C}$ whereas the temperature range of $21^{\circ} \mathrm{C}-30^{\circ} \mathrm{C}$ is the optimum temperatures for the development and survival of western corn rootworm (Jackson and Elliott 1988). The development time for males typically is a little shorter compared to that of females (Jackson and Elliott 1988, Ludwick et al. 2017b). At $24^{\circ} \mathrm{C}$, development time for males from hatch to adult emergence is 26.3 days while that of females is 28.9 days when larvae reared on corn roots (Jackson and Elliott 1998). For males, the development time is $4.8,4.3$, and 9.4 days for $1^{\text {st }}, 2^{\text {nd }}$ and $3^{\text {rd }}$ instar larvae, respectively whereas the development time for females is $5.3,4.9,10.4$ days for $1^{\text {st }}, 2^{\text {nd }}$ and $3^{\text {rd }}$ instar larvae, respectively (Jackson and Elliott 1998). Head capsules width is 200-260, 300-400, and 440-560 $\mu \mathrm{m}$ in width for $1^{\text {st }}, 2^{\text {nd }}$ and $3^{\text {rd }}$ instar larvae, respectively (Hammack et al. 2003). Pupal stages for males and females are 7.8 and 8.4 days, respectively (Jackson and Elliott 1988). Larval dry weight to 10 days ranges from $0.18 \mathrm{mg}$ (Oyediran et al. 2004) to $0.24 \mathrm{mg}$ (Clark and Hibbard 2004) when western corn rootworm larvae are reared on maize under greenhouse conditions, while larval dry weight to 20 days averages between 1.05 mg (Oyediran et al. 2004) and $1.68 \mathrm{mg}$ (Clark and Hibbard 2004). When larvae feed on maize roots, adult head capsule width is $1.23 \mathrm{~mm}$ while adult dry weight is $1.81 \mathrm{mg}$ (Oyediran et al. 2004).

Corn rootworm larvae are subterranean and nearly monophagous on maize roots and can survive on the roots of a limited number of grasses (Krysan and Branson 1983, Clark and Hibbard 2004, Oyediran et al. 2004, Oyediran et al. 2008). The larval stage is responsible for most of the damage associated with corn rootworms. Yield reduction can result from larval 
feeding injury that can cause disruption to water and nutrient flow (Kahler et al. 1985), facilitation of pathogen susceptibility (Palmer and Kommedahl 1969, Kurtz et al. 2010) and reduction of structural stability that can lead the plant lodging, making it difficult to harvest the lodged plants (Spike and Tollefson 1988, 1989, 1991). Adult feeding on leaves, pollen, and silks of maize plants does not usually cause economic losses, but adult populations at high densities prior to and during early anthesis may cause yield reduction by clipping ear silks resulting in a decrease in pollination (Culy et al. 1992). Western corn rootworms are protandrous wherein males typically emerge 5 to 6 days before females in the field (Branson 1987, Quiring and Timmins 1990, Hughson and Spencer 2015, Ludwick et al. 2017b).

\section{The development of resistance of corn rootworms}

The management of western corn rootworm and northern corn rootworm has been complicated because they are highly adaptive insects that have developed resistance to most management tactics. These two species have independently developed biotypes resistant to crop rotation where maize is rotated each season with a non-host crop, typically soybean, Glycine $\max ($ L.) Merr. (Chiang 1973). Western corn rootworm females from the rotation-resistant strain lay the eggs in soybean fields in addition to maize (Levine et al. 2002, Gray et al. 2009) and gut microbiota enterotypes of this pest likely contributed to this because they helped beetles overcome defense compounds in soybean leaves (Chu et al. 2013). Northern corn rootworm with resistance to crop rotation exhibit 'extended diapause' where some eggs hatch two or more years after being laid in the soil (Krysan et al. 1986). Since northern corn rootworm larvae die if there is no host plant after hatching, extended diapause allows northern corn rootworm to adapt to local crop rotations. This biotype is present in many pockets throughout the northern corn 
rootworm range. Western corn rootworm has developed resistance to several chemical insecticides including organochloride, organophosphate, carbamate, and pyrethroid pesticides (Ball and Weekman 1962, Meinke et al. 1998, Pereira et al. 2015, Pereira et al. 2017). Northern corn rootworm also developed resistance to cyclodiene soil insecticides (Hamilton 1965). The latest control tools are transgenic maize hybrids expressing insecticidal crystalline toxins from Bacillus thuringiensis (Bt) Berliner. These were recently documented to falter when resistance developed first to Cry3Bb1 and then to other cry proteins (Gassmann et al. 2011, Zukoff et al. 2016, Ludwick et al. 2017a). Since Bt corn targets both western corn rootworm and northern corn rootworm, recent increases in northern corn rootworm populations in Bt-traited fields might suggest a similar adaptation has occurred with specific northern corn rootworm populations to $\mathrm{Bt}$ and/or other pest management strategies.

In an attempt to mitigate the development of resistance to Bt products, insect resistance management plans are required by the United States Environmental Protection Agency (EPA). As part of these plans, monitoring is required to evaluate the development of resistance to each of the Bt products (EPA 2016). Currently, four Bt proteins including Cry3Bb1, Cry34/35Ab1, mCry3A, and eCry3.1Ab are approved by the EPA for commercial use on corn rootworm larvae. Toxicity bioassays using artificial diet (Siegfried et al. 2005) is an important component of monitoring programs to detect the development of resistance of corn rootworm populations (Zukoff et al. 2016). Other methods for resistance monitoring such as on-plant assays are used in conjunction with diet-toxicity assays (Meihls et al. 2008, Nowatzki et al. 2008, Gassmann et al. 2011, Zukoff et al. 2016). 


\section{Corn rootworm diets}

Artificial diets for rearing insects are highly advantageous for resistance monitoring, insect research and educational programs as well as supplementary nutritional sources in diets of other organisms. The artificial diets often have reduced inputs in terms of labor or space (Beck and Stauffer 1950) and reduced time for host plant rearing (Vanderzant et al. 1956). Other logistical benefits include ease of transport, simplification of diet preparation and presentation, minimizing storage space, and low cost of production. The goal of an artificial diet should always be to produce "normal" insects that are behaviorally and physiologically similar to widetype insects (Lapointe et al. 2010b, Cohen 2015).

Diet development for Diabrotica species began with a formulation for southern corn rootworm, Diabrotica undecimpunctata howardi Barber. This species is a generalist feeding on more than 100 different plant species, has a short life cycle, and does not have a diapausing stage. Sutter et al. (1971) modified the Adkisson et al. diet for pink bollworm, Pectinophora gossypeilla (Saunders) (Adkisson et al. 1960) for rearing southern corn rootworm after evaluating southern corn rootworm development on several established diets for phytophagous insects including European corn borer, Ostrinia nubilalis (Hubner) (Guthrie et al. 1965), pink bollworm, tobacco hornworm, Manduca sexta (Johannson), corn earworm, Heliothis zea (Boddie), and cabbage looper, Trichoplusia ni (Hubner) (Ignoffo 1963). The initial formulation for southern corn rootworm was modified from the Adkisson et al. diet for pink bollworm by changing the agar source and reducing the amount of agar. This agar-based diet with wheat germ and casein as the major nutritional components supported development from egg to adult with no difference in morphology, egg production and viability, but development of southern corn rootworm was slower than when reared on maize roots (Sutter et al. 1971). Rose and McCabe 
(1973) improved the Sutter et al. diet by changing the concentration of diet ingredients and substituting linseed oil with corn oil. This modification resulted in a formulation that supported better larval development compared to southern corn rootworm larvae reared on the Sutter et al. diet, but the Rose and McCabe diet was inferior to maize roots for larval developmental rates (Rose and McCabe 1973).

Marrone et al. (1985) developed an improved diet for southern corn rootworm by changing concentration of ingredients in the Sutter et al. diet (Sutter et al. 1971). The modifications included an increase in raw linseed oil and sucrose, and a decrease in antimicrobial agents and potassium hydroxide. The diet improvement was made based on the evaluation of the effect of diet ingredients on larval performance by varying independently each ingredient in the Sutter et al. diet from $10 \%$ to $100 \%$. Additionally, several additional ingredients (i.e., corn meal, fresh zucchini, lyophilized zucchini, cucurbitacin, cucumber extract, fresh corn root, lyophilized corn roots, ground lima, egg albumin, soy protein, stigmasterol, sitosterol, dextrose, and Beck's salt mix) were tested as substitutions for carbohydrate and protein sources and feeding stimulants (Marrone et al. 1985). Changing types of ingredients in the Sutter et al. diet, except for wheat germ, did not result in any improvement in larval growth. The formalin was recorded as the most detrimental ingredient to larval growth, but a decrease in formalin resulted in an increase in bacterial contamination. No difference was seen in susceptibility to a carbamate insecticide between the diet-adapted southern corn rootworm strain and maize strain (Marrone et al. 1985). The Marrone et al diet supported larval development similar to larvae reared on maize roots, but only after six generations of selection of larvae that reared on this formulation (Marrone et al. 1985). 
The first published diet for western corn rootworm developed by Pleau et al. (2002) was a modification of a diet for southern corn rootworm (Marrone et al. 1985). Several different types of diet ingredient sources including carbohydrates (i.e., wheat germ, corn meal, raw soybean meal, and coarse alfalfa meal), protein (i.e., casein, ground lima beans (Phaseolus lunatus L.), ground pinto beans (Phaseolus vulgaris L.), and sodium caseinate), sugars (i.e., Dsucrose, D-trehalose, D-fructose, D-glucose, and honey), oils (i.e., corn oil, peanut oil, wheat germ oil, soybean oil, and linseed oil), sterols (i.e., cholesterol, $\beta$-sitosterol, stigmastanol, and stigmasterol), cellulose, plant adjuvant (i.e., lyophilized corn roots, zucchini, yellow squash, and a mixture of zucchini and yellow squash), formalin, potassium hydroxide, and agar (i.e., Serva agar, Bio-Serv agar, Difco agar, and Sigma agarose) were tested independently in each nutritional group with the southern corn rootworm diet (Pleau et al. 2002). Several important relationships between diet ingredients and western corn rootworm larval development were seen when varying the diet for western corn rootworm. Adding host plant material (lyophilized corn roots) had a positive effect on larval growth while formalin exhibited a negative impact on larval performance. The negative impact of formalin was also documented in the formulation for southern corn rootworm (Sutter et al. 1971, Marrone et al. 1985). The type of agar used in the diet also had an impact on larval development. Optimum dietary $\mathrm{pH}$ for southern corn rootworm and western corn rootworm also differed. Pleau et al. (2002) discovered that western corn rootworm larvae performed better on a more basic diet with a $\mathrm{pH}$ of 9.0 while southern corn rootworm grew better under more acidic conditions. Pleau et al. (2002) found that optimizing $\mathrm{pH}$, removing formalin, increasing wheat germ, reducing linseed oil, and adding corn root powder nearly doubled growth of western corn rootworm larvae compared to larvae reared on the southern corn rootworm diet. 
No diet has been developed specifically for northern corn rootworm. The diet for southern corn rootworm has been used in northern corn rootworm diet bioassays (Oyediran et al. 2016) while several proprietary diet formulations that were modifications of a diet for western corn rootworm (Pleau et al. 2002) and southern corn rootworm (Marrone et al. 1985) have been developed by each of the major maize seed companies. The diets for corn rootworm are mainly used in toxicity bioassays to detect decreases in susceptibility of western corn rootworm and northern corn rootworm populations to Bt proteins. Since differences in diet formulations can lead to different results in diet toxicity assays (Deans et al. 2017), results generated from toxicity bioassays using different diets cannot be directly compared between Cry toxins. Research with lepidopteran species has demonstrated the effect of nutrition on the toxicity of Bt proteins (Janmaat and Myers 2005, Bird and Akhurst 2007, Raymond et al. 2007, Blanco et al. 2009, Orpet et al. 2015). In fact, the susceptibility of cabbage looper, Trichoplusia ni Hübner, to Cry1Ac was different when larvae fed on diets differing in the ratio of protein: carbohydrate (Orpet et al. 2015). If the nutrition plays a role in the intoxication of rootworms to Bt proteins, it is impossible to make diet comparisons between toxicity assays using different artificial diets from individual companies. Thus, an accurate picture of the test population's phenotype as it relates to Bt resistance may be not determined.

Contamination is one of the limiting issues in diet bioassays and continuous rearing for corn rootworms (Sutter et al. 1971, Rose and McCabe 1973, Marrone et al. 1985, Pleau et al. 2002, Pereira et al. 2016). Several efforts have been made to reduce the contamination. Initially, several diet preservatives (e.g., streptomycin, chlortetracycline, sorbic acid, methylparaben) and formalin were added to the diet to control the microbial contamination (bacteria and fungi) (Sutter et al. 1971, Rose and McCabe 1973, Marrone et al. 1985). Marrone et al. (1985) 
improved rearing conditions using an egg washing process including several disinfectants and peracetic acid that resulted in greatly reduced bacterial contamination from surface pits on the eggs. The surface disinfestation for egg washing was improved by Pleau et al. (2002) using Lysol $^{\circledR}$ disinfectants and $10 \%$ formalin for $3 \mathrm{~min}$. This allowed the removal of formalin from the diet formulations and eliminated the negative impact of formalin on larval development when added to the diet. Later, several modified diet formulations were made by industry with high loads of antibiotics that were added into the diet for southern corn rootworm to reduce contamination (Zhao et al. 2016).

The diet toxicity bioassays for western corn rootworm and northern corn rootworm typically run up to 7 days. The microbial contamination has been a major issue (Pereira et al. 2016) that can force diet bioassays to be terminated early, limiting further observations. Data recorded at 7 days can include two life history parameters including larval survival and weight, but ignores other developmental traits such as molting. Improving bioassay techniques to extend observations for longer periods would allow evaluating several life history parameters (e.g., survival, molting, and weight) on multiple developmental stages. Also, the bioassays with longer periods would be beneficial for testing products with delayed activity (e.g. dsRNA).

\section{Diet development methodologies}

Insect diets are mixtures of several diet ingredients including nutritional sources such as carbohydrates, proteins, lipids, vitamins and minerals. Other common ingredients added to the diet are diet preservatives (antimicrobial agents and antioxidants), gelling agents, stabilizers or emulsifiers. For many insects, natural host plant or token stimuli are also added to make the diets more desirable to insects. Corn roots contain both host recognition cues for western corn 
rootworm (Bernklau et al. 2015) and feeding cues that are a combination of three sugars, glucose: fructose: sucrose plus one free fatty acid (oleic or linoleic acid) (Bernklau and Bjostad 2008), but also consist of a repellent for western corn rootworm (Bernklau et al. 2016).

The diet formulation approaches for developing artificial diets for southern corn rootworm and western corn rootworm discussed above were based on the identification of the effect of individual diet ingredients on insect performance. Typically, the diet components were changed one at a time and life history parameters were used to assess the effect of each change. Changing one ingredient at a time is suitable for determining the effect of a single diet component, but ignores the interaction between diet ingredients. Since diet formulations consist of several ingredients, the interactive effects between the ingredients are important for identification of the optimal blends of diet components necessary to maximize the measured responses of interest. Changing one ingredient at time was not able to elucidate the interactive effects between diet components, so this approach was less likely to identify optimal blends of diet mixtures (Niedz and Evens 2016).

The evaluation of mixtures is constrained because the proportions of all components (diet ingredients in this case) always add up to 1 (unity). Formulation modification based on a single ingredient has zero dimensions because the mixture cannot be varied. A 2-component blend has a single dimension that can be envisioned as a line with the end points corresponding to pure blends of the individual components. A 3-component blend can be represented by a 2dimensional triangle using a ternary plot to represent all possible blend combinations. Thus, the design space of any mixture experiment is necessarily of $n-1$ dimensions where $n$ is the number of mixture components. Most importantly, it is imperative to understand that the amount of any diet ingredient, properly referred to as its proportion, cannot be varied independently. When the 
amount of any ingredient is varied, the proportion of all remaining ingredients is also changed. Scheffé (1958) developed mathematical models specifically for mixture experiments. In Scheffé models, the total amount of the mixture components remains constant. Later, Piepel and Cornell (1987) improved the mixture experiment that allows the total amount of the mixture in the Scheffé polynomials to vary.

The application of mathematical or geometric models has been shown to be of great value for insect diet research. Lee et al. (2008) applied response surface modeling to identify the relationships between ratio of carbohydrate: protein and lifespan and fecundity of fruit fly, Drosophila melanogaster Meigen, while Tan et al. (2013) developed an improved diet for the predator, Orius sauteri (Poppius), using orthogonal experimental designs. Furthermore, the application of response surface methodology combined with mixture experiments has been shown to be of great value for insect diet improvement and optimization (Lapointe et al. 2008, Lapointe et al. 2010a, Pascacio-Villafán et al. 2014) where factorial designs are not well-suited to deal with the unique aspects of mixture experimentation.

Response surface methodology is applied extensively in industry (e.g., biotechnology, pharmaceuticals, chemical and process industries) to design, develop, and formulate new products or to improve existing product designs (Myers et al. 2016). Response surface methodology consists of a collection of mathematical and statistical techniques that can be applied to study functional relationships between a number of variables and responses of interest (Khuri and Mukhopadhyay 2010) to determine important factors responsible for improved desirable traits and to identify the optimum level of selective variables resulting in the maximum targeted response over a region of interest (Myers et al. 2016). Furthermore, modern multivariate geometric designs for mixtures can eliminate the problems of traditional factorial designs when 
applied to multiple diet components (Lapointe et al. 2008). The traditional method to determine the effect of varying the doses of several components requires large factorial experiments resulting in a huge numbers of treatment combinations and parallel variation of variables, making it difficult to interpret the results and inappropriate for diet formulations as mixtures where diet components (e.g. carbohydrates, lipids, proteins, vitamins, preservatives) are used (Ruohonen and Kettunen 2004).

Lapointe et al. (2008) proposed an iterative approach to improve diet formulations using mixture experiments. First, an initial screening experiment (Cornell 2002) is performed to screen a large number of ingredients to aid identification of diet ingredients (key ingredients) that are more important to life history parameters. Once the key ingredients are identified, mixture experiments are conducted to optimize the key ingredients that maximize the desirable developmental traits of interest. Mathematical equations are generated to describe the relationships between diet components and insect responses that can be used to predict optimum formulations. Confirmatory experiments might be needed to validate the new formulations that match the goals of interest. 


\section{References}

Adkisson, P. L., E. S. Vanderzant, D. L. Bull, and W. E. Allison. 1960. A wheat germ medium for rearing the pink bollworm. J. Econ. Entomol. 53: 759-762.

Bača, F. 1994. New member of the harmful entomofauna of Yugoslavia Diabrotica virgifera virgifera LeConte (Coleoptera: Chrysomelidae). Zaštita bilja 45: 125-131.

Ball, H. J. 1957. On the biology and egg-laying habits of the western corn rootworm. J. Econ. Entomol. 50: 126-128.

Ball, H. J., and G. T. Weekman. 1962. Insecticide resistance in adult western corn rootworm in Nebraska. J. Econ. Entomol. 55: 439-441.

Beck, S. D., and J. F. Stauffer. 1950. An aseptic method for rearing European corn borer larvae. J. Econ. Entomol. 43: 4-6.

Bernklau, E. J., and L. B. Bjostad. 2008. Identification of feeding stimulants in corn roots for western corn rootworm (Coleoptera: Chrysomelidae) larvae. J. Econ. Entomol. 101: 341351.

Bernklau, E. J., B. E. Hibbard, A. P. Norton, and L. B. Bjostad. 2016. Methyl anthranilate as a repellent for western corn rootworm larvae (Coleoptera: Chrysomelidae). J. Econ. Entomol. 109: 1683-1690.

Bernklau, E. J., B. E. Hibbard, D. L. Dick, C. D. Rithner, and L. B. Bjostad. 2015. Monogalactosyldiacylglycerols as host recognition cues for western corn rootworm larvae (Coleoptera: Chrysomelidae). J. Econ. Entomol. 108: 539-548.

Bird, L. J., and R. J. Akhurst. 2007. Effects of host plant species on fitness costs of Bt resistance in Helicoverpa armigera (Lepidoptera: Noctuidae). Biol. Control 40: 196-203.

Blanco, C. A., F. Gould, P. Vega-Aquino, J. L. Jurat-Fuentes, O. P. Perera, and C. A. Abel. 2009. Response of Heliothis virescens (Lepidoptera: Noctuidae) strains to Bacillus thuringiensis Cry1 Ac incorporated into different insect artificial diets. J. Econ. Entomol. 102: 1599-1606. 
Branson, T. F., and J. L. Krysan. 1981. Feeding and oviposition behavior and life cycle strategies of Diabrotica: an evolutionary view with implications for pest management. Environ. Entomol. 10: 826-831.

Branson, T. F. 1987. The contribution of prehatch and posthatch development to protandry in the chrysomelid, Diabrotica virgifera virgifera. Ent. Exp. Appl. 43: 205-208.

CABI. 2017. Diabrotica virgifera virgifera (western corn rootworm) [distribution maps]. CABI, Invasive Species Compendium.

Chiang, H. C. 1973. Bionomics of the northern and western corn rootworms. Annu. Rev. Entomol. 18: 47-72.

Chu, C., J. L. Spencer, M. J. Curzi, J. A. Zavala, and M. J. Seufferheld. 2013. Gut bacteria facilitate adaptation to crop rotation in the western corn rootworm. Proc. Nat. Acad. Sci. 110: 11917-11922.

Clark, T. L., and B. E. Hibbard. 2004. Comparison of nonmaize hosts to support western corn rootworm (Coleoptera : Chrysomelidae) larval biology. Environ. Entomol. 33: 681-689.

Cohen, A. C. 2015. Insect diets: science and technology, 2nd ed., Taylor \& Francis Group.

Cornell, J. A. 2002. Experiments with mixtures: designs, models, and the analysis of mixture data, 3rd ed. John Wiley \& Sons, Inc., New York, USA.

Culy, M. D., C. R. Edwards, and J. R. Cornelius. 1992. Effect of silk feeding by western corn rootworm (Coleoptera: Chrysomelidae) on yield and quality of inbred corn in seed corn production fields. J. Econ. Entomol. 85: 2440-2446.

Deans, C. A., S. T. Behmer, A. E. Tessnow, P. Tamez-Guerra, M. Pusztai-Carey, and G. A. Sword. 2017. Nutrition affects insect susceptibility to Bt toxins. Sci. Rep. 7: 39705.

EPA. 2016. EPA docket for corn rootworm resistance management and framework for Bt corn. In U. S. E. P. Agency [ed.]. United States Environmental Protection Agency, Washington, DC, USA.

Gassmann, A. J., J. L. Petzold-Maxwell, R. S. Keweshan, and M. W. Dunbar. 2011. Fieldevolved resistance to Bt maize by western corn rootworm. PloS one 6: e22629. 
Gray, M. E., T. W. Sappington, N. J. Miller, J. Moeser, and M. O. Bohn. 2009. Adaptation and invasiveness of western corn rootworm: intensifying research on a worsening pest. Annu. Rev. Entomol. 54: 303-321.

Guthrie, W., E. Raun, F. Dicke, G. Pesho, and S. Carter. 1965. Laboratory production of European corn borer egg masses. Iowa State J. Sci. 40: 65-83.

Hamilton, E. W. 1965. Aldrin resistance in corn rootworm beetles. J. Econ. Entomol. 58: 296300.

Hammack, L., M. M. Ellsbury, R. L. Roehrdanz, and J. Pikul Jr. 2003. Larval sampling and instar determination in field populations of northern and western corn rootworm (Coleoptera: Chrysomelidae). J. Econ. Entomol. 96: 1153-1159.

Hughson, S. A., and J. L. Spencer. 2015. Emergence and abundance of western corn rootworm (Coleoptera: Chrysomelidae) in Bt cornfields with structured and seed blend refuges. J. Econ. Entomol. 108: 114-125.

Ignoffo, C. M. 1963. A successful technique for mass-rearing cabbage loopers on a semisynthetic diet. Ann. Entomol. Soc. Am. 56: 178-182.

Jackson, J. J., and N. C. Elliott. 1988. Temperature-dependent development of immature stages of the western corn rootworm, Diabrotica virgifera virgifera (Coleoptera: Chrysomelidae). Environ. Entomol. 17: 166-171.

Janmaat, A. F., and J. H. Myers. 2005. The cost of resistance to Bacillus thuringiensis varies with the host plant of Trichoplusia ni. Proc. R. Soc. London B: Biological Sciences 272: 1031-1038.

Kahler, A., A. Olness, G. Sutter, C. Dybing, and O. Devine. 1985. Root damage by western corn rootworm and nutrient content in maize. Agron. J. 77: 769-774.

Khuri, A. I., and S. Mukhopadhyay. 2010. Response surface methodology. WIREs. Comp. Stats. 2: $128-149$.

Krysan, J., and T. Branson. 1977. Inheritance of diapause intensity in Diabrotica virgifera. J. Heredity 68: 415-417. 
Krysan, J., and T. Branson. 1982. Biology, ecology, and distribution of Diabrotica. In, International Maize Virus Disease Colloquium and Workshop, Wooster, Ohio (USA), 2-6 Aug 1982. Ohio Agricultural Research and Development Center.

Krysan, J., D. Foster, T. Branson, K. Ostlie, and W. Cranshaw. 1986. Two years before the hatch: rootworms adapt to crop rotation. Bull. ESA 32: 250-253.

Krysan, J. L. 1986. Introduction: biology, distribution, and identification of pest Diabrotica. In, Methods for the study of pest Diabrotica (ed. by J. L. Krysan and T.A. Miller), pp. 1-23, Springer-Verlag, New York.

Kurtz, B., P. Karlovsky, and S. Vidal. 2010. Interaction between western corn rootworm (Coleoptera: Chrysomelidae) larvae and root-infecting Fusarium verticillioides. Environ. Entomol. 39: 1532-1538.

Lapointe, S. L., T. J. Evens, and R. P. Niedz. 2008. Insect diets as mixtures: Optimization for a polyphagous weevil. J. Ins. Physiol. 54: 1157-1167.

Lapointe, S. L., R. P. Niedz, and T. J. Evens. 2010a. An artificial diet for Diaprepes abbreviatus (Coleoptera: Curculionidae) optimized for larval survival. Fla. Entomol. 93: 56-62.

Lapointe, S. L., T. J. Evens, R. P. Niedz, and D. G. Hall. 2010b. Artificial diet optimized to produce normative adults of Diaprepes abbreviatus (Coleoptera: Curculionidae). Environ. Entomol. 39: 670-677.

Lee, K. P., S. J. Simpson, F. J. Clissold, R. Brooks, J. W. Ballard, P. W. Taylor, N. Soran, and D. Raubenheimer. 2008. Lifespan and reproduction in Drosophila: New insights from nutritional geometry. Proc. Nat. Acad. Sci. 105: 2498-2503.

Levine, E., and H. Oloumi-Sadeghi. 1991. Management of diabroticite rootworms in corn. Annu. Rev. Entomol. 36: 229-255.

Levine, E., J. L. Spencer, S. A. Isard, D. W. Onstad, and M. E. Gray. 2002. Adaptation of the western corn rootworm to crop rotation: evolution of a new strain in response to a management practice. Am. Entomol. 48: 94-117.

Ludwick, D. C., L. N. Meihls, K. R. Ostlie, B. D. Potter, L. French, and B. E. Hibbard. 2017 a. Minnesota field population of western corn rootworm (Coleoptera: Chrysomelidae) 
shows incomplete resistance to Cry34Ab1/Cry35Ab1 and Cry3Bb1. J. Appl. Entomol. 141: $28-40$.

Ludwick, D. C., A. Zukoff, M. Higdon, and B. E. Hibbard. 2017b. Protandry of western corn rootworm (Coleoptera: Chrysomelidae) beetle emergence partially due to earlier egg hatch of males. J. Kan. Entomol. Soc. 90: 94-99.

Marrone, P. G., F. D. Ferri, T. R. Mosley, and L. J. Meinke. 1985. Improvements in laboratory rearing of the southern corn rootworm, Diabrotica undecimpuncta howardi Barber (Coleoptera: Chrysomelidae), on an artificial diet and corn. J. Econ. Entomol.78: 290293.

Meihls, L. N., M. L. Higdon, B. D. Siegfried, N. J. Miller, T. W. Sappington, M. R. Ellersieck, T. A. Spencer, and B. E. Hibbard. 2008. Increased survival of western corn rootworm on transgenic corn within three generations of on-plant greenhouse selection. Proc. Nat. Acad. Sci. 105: 19177-19182.

Meinke, L. J., B. D. Siegfried, R. J. Wright, and L. D. Chandler. 1998. Adult susceptibility of Nebraska western corn rootworm (Coleoptera: Chrysomelidae) populations to selected insecticides. J. Econ. Entomol. 91: 594-600.

Metcalf, R. L. 1986. Methods for the study of pest Diabrotica (ed. by J. L. Krysan and T.A. Miller), pp. vii-xv, Springer-Verlag, New York.

Mitchell, P. D. 2011. Costs and benefits of controlling pest Diabrotica in maize in the United States. In, 24th IWGO Conference and 3rd International Conference of Diabrotica Genetics, IWGO, Freiburg, Germany.

Murphy, A. F., M. D. Ginzel, and C. H. Krupke. 2010. Evaluating western corn rootworm (Coleoptera: Chrysomelidae) emergence and root damage in a seed mix refuge. J. Econ. Entomol. 103: 147-157.

Myers, R. H., D. C. Montgomery, and C. M. Anderson-Cook. 2016. Response surface methodology: process and product optimization using designed experiments, 4th ed., John Wiley \& Sons, New York, NY, USA.

Niedz, R. P., and T. J. Evens. 2016. Design of experiments (DOE)—history, concepts, and relevance to in vitro culture. In Vitro Cell. Dev. Biol. - Plant 52: 547-562. 
Nowatzki, T. M., S. A. Lefko, R. R. Binning, S. D. Thompson, T. A. Spencer, and B. D. Siegfried. 2008. Validation of a novel resistance monitoring technique for corn rootworm (Coleoptera: Chrysomelidae) and event DAS-59122-7 maize. J. Appl. Entomol. 132: 177188.

Orpet, R. J., B. A. Degain, G. C. Unnithan, K. L. Welch, B. E. Tabashnik, and Y. Carrière. 2015. Effects of dietary protein to carbohydrate ratio on Bt toxicity and fitness costs of resistance in Helicoverpa zea. Entomol. Exp. Appl. 156: 28-36.

Oyediran, I. O., B. E. Hibbard, and T. L. Clark. 2004. Prairie grasses as hosts of the western corn rootworm (Coleoptera : Chrysomelidae). Environ. Entomol. 33: 740-747.

Oyediran, I. O., B. W. French, T. L. Clark, K. E. Dashiell, and B. E. Hibbard. 2008. Prairie grasses as hosts of the northern corn rootworm (Coleoptera: Chrysomelidae). Environ. Entomol. 37: 247-254.

Oyediran, I. O., P. Matthews, N. Palekar, W. French, J. Conville, and T. Burd. 2016. Susceptibility of northern corn rootworm Diabrotica barberi (Coleoptera: Chrysomelidae) to mCry3A and eCry3.1Ab Bacillus thuringiensis proteins. J. Insect Sci. 23: 913-917.

Palmer, L., and T. Kommedahl. 1969. Root-infecting Fusarium species in relation to rootworm infestations in corn. Phytopathology. 59:1613-1617

Pascacio-Villafán, C., S. Lapointe, T. Williams, J. Sivinski, R. Niedz, and M. Aluja. 2014. Mixture-amount design and response surface modeling to assess the effects of flavonoids and phenolic acids on developmental performance of Anastrepha ludens. J. Chem. Ecol. 40: 297-306.

Pereira, A. E., N. P. Carneiro, and B. D. Siegfried. 2016. Comparative susceptibility of southern and western corn rootworm adults and larvae to vATPase-A and Snf7 dsRNAs. J. RNAi and Gene Silencing 12: 528-535

Pereira, A. E., D. Souza, S. N. Zukoff, L. J. Meinke, and B. D. Siegfried. 2017. Cross-resistance and synergism bioassays suggest multiple mechanisms of pyrethroid resistance in western corn rootworm populations. PloS one: 12(16): e0179311. 
Pereira, A. E., H. Wang, S. N. Zukoff, L. J. Meinke, B. W. French, and B. D. Siegfried. 2015. Evidence of field-evolved resistance to bifenthrin in western corn rootworm (Diabrotica virgifera virgifera LeConte) populations in western Nebraska and Kansas. PloS one 10: e0142299.

Piepel, G. F., and J. A. Cornell. 1987. Designs for mixture-amount experiments. J. Qual. Tech. 19: 11-28.

Pleau, M. J., J. E. Huesing, G. P. Head, and D. J. Feir. 2002. Development of an artificial diet for the western corn rootworm. Entomol. Exp. Appl. 105: 1-11.

Quiring, D. T., and P. R. Timmins. 1990. Influence of reproductive ecology on feasibility of mass trapping Diabrotica virgifera virgifera (Coleoptera: Chrysomelidae). J. Appl. Ecol. 27: 965-982.

Raymond, B., A. H. Sayyed, and D. J. Wright. 2007. Host plant and population determine the fitness costs of resistance to Bacillus thuringiensis. Biol. Lett. 3: 83-86.

Rose, R. I., and J. M. McCabe. 1973. Laboratory rearing techniques for the southern corn rootworm. J. Econ. Entomol. 66: 398-400.

Ruohonen, K., and J. Kettunen. 2004. Effective experimental designs for optimizing fish feeds. Aquacult. Nutr. 10: 145-151.

Scheffé, H. 1958. Experiments with mixtures. Royal Stat. Soc., Series B (Methodological) 20: 344-360.

Siegfried, B. D., T. T. Vaughn, and T. Spencer. 2005. Baseline susceptibility of western corn rootworm (Coleoptera: Crysomelidae) to Cry3Bb1 Bacillus thuringiensis toxin. J. Econ. Entomol. 98: 1320-1324.

Spike, B. P., and J. J. Tollefson. 1988. Western corn rootworm (Coleoptera: Chrysomelidae) larval survival and damage potential to corn subjected to nitrogen and plant density treatments. J. Econ. Entomol. 81: 1450-1455.

Spike, B. P., and J. J. Tollefson. 1989. Relationship of plant phenology to corn yield loss resulting from western corn rootworm (Coleoptera: Chrysomelidae) larval injury, nitrogen deficiency, and high plant density. J. Econ. Entomol. 82: 226-231. 
Spike, B. P., and J. J. Tollefson. 1991. Yield response of corn subjected to western corn rootworm (Coleoptera: Chrysomelidae) infestation and lodging. J. Econ. Entomol. 84: 1585-1590.

Sutter, G. R., J. L. Krysan, and P. L. Guss. 1971. Rearing the southern corn rootworm on artificial diet. J. Econ. Entomol. 64: 65-67.

Tan, X. L., S. Wang, and F. Zhang. 2013. Optimization an optimal artificial diet for the predatory bug Orius sauteri (Hemiptera: Anthocoridae). PloS one 8: e61129.

Vanderzant, E. S., R. Reiser, and E. Ivy. 1956. Methods for the mass rearing of the pink bollworm. J. Econ. Entomol. 49: 559-560.

Wesseler, J., and E. H. Fall. 2010. Potential damage costs of Diabrotica virgifera virgifera infestation in Europe-the 'no control'scenario. J. Appl. Entomol. 134: 385-394.

Wilcox, J. 1972. Coleopterorum Catalogus Supplementa (Chrysomelidae: Galerucinae, Luperini: Aulacophorina, Diabroticina), pars 78, fascicle 2. Gravenhage, The Netherlands: Dr W. Junk.

Zhao, J. Z., M. A. Oneal, N. M. Richtman, S. D. Thompson, M. C. Cowart, M. E. Nelson, Z. Pan, A. P. Alves, and T. Yamamoto. 2016. mCry3A-selected western corn rootworm (Coleoptera: Chrysomelidae) colony exhibits high resistance and has reduced binding of mCry3A to midgut tissue. J. Econ. Entomol. 109: 1369-1377.

Zukoff, S. N., K. R. Ostlie, B. Potter, L. N. Meihls, A. L. Zukoff, L. French, M. R. Ellersieck, B. Wade French, and B. E. Hibbard. 2016. Multiple assays indicate varying levels of cross resistance in Cry3Bb1-selected field populations of the western corn rootworm to mCry3A, eCry3.1Ab, and Cry34/35Ab1. J. Econ. Entomol. 109: 1387-1398. 


\title{
CHAPTER II: DIET IMPROVEMENT FOR WESTERN CORN ROOTWORM LARVAE*
}

\begin{abstract}
The western corn rootworm (WCR), Diabrotica virgifera virgifera LeConte, is the most serious insect pest of corn (Zea mays L.) in the United States and parts of Europe, and arguably one of the world's most expensive pests to control. Several diet formulations are currently used by industry and public researchers to evaluate WCR larvae in diet-toxicity bioassays. However, a publicly available diet that produces normative insects that are physiologically similar to WCR larvae reared on corn roots will accelerate development of management technologies. We report a new diet formulation that supports improved weight gain, larval development and survival compared with the only public diet for WCR that is currently available in the refereed literature. The formulation was created by using response surface methods combined with n-dimensional mixture designs to identify and improve the formulation of key ingredients. Weight gain increased two-fold, and survival and molting rates increased from $93 \%$ and $90 \%$, respectively when reared on the public diet, to approximately $99 \%$ for both survival and molting at 11 days when reared on our new formulation. This new formulation provides a standardized growth medium for WCR larvae that will facilitate comparison of research results from various working groups and compliance with regulatory requirements.
\end{abstract}

\footnotetext{
* This chapter appeared in PloS one. Huynh M. P., L. N. Meihls, B. E. Hibbard, S. L. Lapointe, R. P. Niedz, D. C. Ludwick, and T. A. Coudron. 2017. Diet improvement for western corn rootworm (Coleoptera: Chrysomelidae) larvae. PloS one 12: e0187997. doi:10.1371/journal.pone.0187997.
} 


\section{Introduction}

The western corn rootworm (WCR), Diabrotica virgifera virgifera LeConte, is the most serious insect pest of maize in the United States and parts of Europe. The larval stage feeds primarily on maize roots and is responsible for the majority of damage caused by WCR. Adult WCR feed on the green silks of maize plants and may cause yield reduction at high densities if present prior to anthesis (Culy et al. 1992). Crop losses and abatement efforts to minimize damage by corn rootworm have been estimated to reach $\$ 2$ billion annually (Mitchell 2011). Management of WCR has continuously presented a challenge to industry and corn growers because WCR has developed resistance to management tactics including chemical insecticides (Ball and Weekman 1963, Meinke et al. 1998, Pereira et al. 2015), crop rotation (Levine et al. 2002, Gray et al. 2009), and transgenic maize which expresses insecticidal proteins derived from Bacillus thuringiensis (Bt) (Gassmann et al. 2011). Presently, some level of field-evolved resistance has developed to all single-gene Bt proteins currently available for WCR management (Gassmann et al. 2016, Zukoff et al. 2016, Ludwick et al. 2017).

Research efforts to avert resistance development require some level of ability to rear WCR. Although a standardized rearing method for research and regulatory purposes is highly preferred (EPA 2016a), a standardized method has not been adopted for WCR and consequently both plant and diet rearing systems are currently used for bioassays. Life history parameters and toxicity results differ greatly across these systems, making comparisons difficult and limiting the extent to which results can be interpreted (Ludwick and Hibbard 2016). An artificial diet that supports normative development of WCR larvae (i.e., similar to that of WCR reared on maize root) would be a major advancement in achieving a standardized bioassay method. 
The first artificial diet for Diabrotica species was formulated for the southern corn rootworm (SCR), Diabrotica undecimpunctata howardi Barber. The ease of rearing SCR made it an attractive substitute for WCR in experiments (Sutter et al. 1971). This agar-based diet with wheat germ and casein as the major nutritional components supported development from egg to adult but resulted in slower development and reduced fecundity compared to those reared on corn roots. Subsequent changes to the formulation (Rose and McCabe 1973, Marrone et al. 1985) increased raw linseed oil and sucrose, and decreased antimicrobial agents and potassium hydroxide. These changes resulted in a diet that supported developmental rates for SCR that were similar to larvae reared on corn roots, but only after 6 generations of rearing on the diet, which suggests some formulation improvements were still possible.

Pleau et al. (2002) formulated the only published diet for rearing WCR larvae, which was a modification of diets developed for SCR (Sutter et al. 1971, Marrone et al. 1985). Several important differences were noted when adjusting the diet for WCR. Optimizing the $\mathrm{pH}$, removing formalin and adding corn root powder nearly doubled the weight of WCR larvae compared with the SCR formulation (Pleau et al. 2002). This diet has been used in a diet-toxicity bioassay to evaluate baseline susceptibility of various WCR populations to a Cry3Bb1 toxin (Siegfried et al. 2005). However, for a WCR diet to achieve its full potential of fulfilling the needs of researchers, regulatory agencies and industry, improvements will have to support diet bioassays sensitive enough to detect differences between susceptible and resistant insects, to support molting, to be publicly available, and ultimately, to obtain development similar to when WCR is reared on corn roots (EPA 2014, 2016b, Ludwick and Hibbard 2016).

Formulation efforts for improving the artificial diet for SCR and WCR discussed above measured biochemical and/or physiological parameters to test the effect of changes in diet 
formulation on insect performance. Typically, diet components were changed one at a time and insect performance was assessed after each change following a lengthy feeding period. This traditional method to determine the effect of varying the doses of several components requires large factorial experiments resulting in a huge numbers of treatment combinations and parallel variation of variables, making it difficult to interpret the results (Ruohonen and Kettunen 2004). However, the process of changing one-ingredient-at-a-time (OIAT) within a mixture is not capable of identifying an optimal blend of the ingredients because the effects of proportion and amount are confounded in factorial designs (Niedz and Evens 2016). When the proportion of any ingredient is varied, the proportions of all remaining ingredients are also changed. For this reason, mathematical models were developed by Henri Scheffé (1958) to deal specifically with the problem of modeling responses to mixtures, now commonly referred to as Scheffé models or Scheffé polynomials. Response surface modeling (RSM) and advanced software (Anderson and Whitcomb 2004, Lapointe et al. 2008) have simplified the corresponding computations. The application of RSM based on multivariate geometric design for mixtures (Lapointe et al. 2008, Lapointe et al. 2010b, Pascacio-Villafán et al. 2014, Cohen 2015) is of great value for insect diet improvement and optimization.

Other insect diet formulations have been improved using multi-dimensional mixture designs (Lapointe et al. 2008) to help identify ingredients (i.e, key ingredients) that have the greatest effect on insect development. A mixture experiment used to formulate a diet is a special type of response surface experiment (Lapointe et al. 2008, Myers et al. 2016) in which ingredients are mixture components as input variables, and life history traits as response measures. Mathematical equations can be derived that predict the impact of diet components on insect responses and in that manner used to generate formulation improvements. Validation 
experiments are conducted to verify that the new formulations result in the predicted performance improvements.

The objective of this research was to optimize the composition of ingredients in the Pleau et al. diet. The approach used multivariate geometric mixture designs combined with response surface modeling (Lapointe et al. 2008) to identify and evaluate the proportion of the key components in the diet for improved larval performance (larval survival, development, and weight) while limiting diet contamination.

\section{Materials and methods}

\section{Insects}

WCR eggs (non-diapausing strain) were obtained from the USDA-ARS Plant Genetics Research Unit at Columbia, Missouri, in a Petri dish with 70 mesh sieved soil and incubated at a constant $25^{\circ} \mathrm{C}$ in darkness. After several larvae were observed to hatch, the remaining eggs were washed out of the soil using a technique described by Pleau et al. (2002). The soil was washed through a 60 mesh sieve with water. The remaining eggs were surfacetreated first with undiluted lysol ${ }^{\circledR}$ (Reckitt Benckiser, LLC, Parsippany, NJ) for 3 min and then triple rinsed with distilled water. Next, the eggs were treated with $10 \%$ formalin (product \# HT501128, Sigma Aldrich, St. Louis, MO) for 3 min and then triple rinsed with distilled water. The eggs were pipetted onto a coffee filter paper (Pure Brew, Rockline Industries, Sheboygan, WI) held inside a 16 oz. Solo ${ }^{\circledR}$ deli cup with a plastic lid (product \#LG8RB-0090 and \#DM16R0090, Solo Cup Company, Lake Forest, IL) containing 5 to 6 vent holes made by a number zero insect pin, and incubated at $25^{\circ} \mathrm{C}$ until hatch. 


\section{Diet preparation}

The diet was prepared using a procedure modified from Pleau et al. (2002). All glassware and containers were sterilized with ca. $0.5 \%$ sodium hypochlorite or exposure to UV light for 5 minutes prior to use. Distilled water and agar (product \#A7002, Sigma-Aldrich) were added to a $400 \mathrm{ml}$ glass beaker. The solution was boiled in a microwave for $2 \mathrm{~min}$ and then poured into a blender (Hamilton Beach, Inc., Model 51101BZ). The agar solution was cooled to $65^{\circ} \mathrm{C}$ and wheat germ (product \#1661, Bio-Serv, Flemington, NJ), casein (product \#1100, BioServ), cellulose (product \#3425, Bio-Serv), sucrose (product \#04821721, MP Biomedicals, Santa Ana, CA), corn root powder (Monsanto, St Louis, MO), salt mix (product \#F8680, Bio-Serv), vitamin mix (product \#V1007, Sigma-Aldrich), methyl paraben (product \#H5501, SigmaAldrich), cholesterol (product \#C8503, Sigma-Aldrich), and sorbic acid (product \#S1626, SigmaAldrich) were added to the blender and mixed thoroughly. The diet was poured into a $750 \mathrm{ml}$ glass beaker containing a stir-bar and placed on a stirring hot plate (Thermo scientific,

Cimarec ${ }^{\mathrm{TM}}$ ). The mixture was held at $65^{\circ} \mathrm{C}$ and slowly stirred while adding other ingredients, i.e., linseed oil (product \#430021, Sigma-Aldrich), wheat germ oil (product \#W1000, SigmaAldrich), streptomycin (612240500, Across, Morris Plains, New Jersey), chlortetracycline (C4881, Sigma-Aldrich). Green food coloring (Bulter, Lancaster, PA) was added to improve visual contrast. The $\mathrm{pH}$ of the diet was adjusted to 9 by adding $10 \% \mathrm{KOH}(\mathrm{w} / \mathrm{v})$ (product \#P250, Fisher Scientific, Fair Lawn, NJ) and monitored with indicator strips (Whatman). The molten diet was dispensed into wells $(200 \mu 1 /$ well) of a 96-well immunoassay plate (product \#3370, Corning Inc., Corning, NY) using a repeater pipette (Eppendorf repeater plus). The diet was placed in a biological safety cabinet (Nuaire, Biological safety cabinet) and allowed to cool and evaporate excess moisture for $30 \mathrm{~min}$. All subsequent diet and insect transfers were done in the 
biological cabinet. Plates were stored in a refrigerator at $4^{\circ} \mathrm{C}$ and used within two weeks. Each formulation was pipetted into a randomly-assigned 12-well row on the plate. Eight different diet solutions were tested per plate. Each formulation was replicated at least 4 times. The response of 12 larvae was averaged and constituted a single replication in the analysis.

\section{Insect bioassays}

Larvae were transferred onto the diet within in a sterile biological safety cabinet within $24 \mathrm{~h}$ of hatching. One larva was placed per well using a No. 1 paintbrush. The plate was covered with a sealing film (Excel scientific, Inc., Thermalseal RTS ${ }^{\mathrm{RM}}$, TSS-RTQ-100) and a single vent hole was made over each well with an insect pin (size 0). The plates were held in the dark at $25^{\circ} \mathrm{C}$. Larvae were checked daily. Surviving larvae from each formulation were collected and pooled within a replication after 7 or 11 days and stored in $95 \%$ ethanol. Collections made at 7 days were used to determine survival and weight differences and to allow comparison of our data with previously published data. Collections made at 11 days were used to determine differences in survival, weight and molting to the $2^{\text {nd }}$ instar. Diet contamination (fungal and bacterial), larval survival, and time of molt to second and third instars were recorded during the bioassay and dry larval weight was determined after the bioassay. For dry larval weight, excess ethanol was discarded, and the remaining ethanol was evaporated and the larvae dried in an oven (Blue M Therm Dry Bacteriological Incubator, Model \#602752) at $50^{\circ} \mathrm{C}$ for two days. Dried larvae were weighed with a micro balance (Sartorius ${ }^{\mathrm{TM}} \mathrm{Cubis}^{\mathrm{TM}}, 6.6 \mathrm{~S}$ ). 


\section{Experimental approach}

An iterative approach was used to identify and optimize critical diet ingredients for specific response variables. An initial screening design (Cornell 2002) was used to identify ingredients with large effects on the measured responses from the 8 components of a previously published diet (Pleau et al. 2002). These ingredients were carried forward to the second phase.

The dimensionality of mixture designs can be expressed as $n-1$ dimensions where $n$ is the number of components (Niedz and Evens 2016). Therefore, the screening design of 8 ingredients is referred to as a 7-dimensional design space. Polynomial equations were generated to describe the impact of important ingredients on larval weight, proportion of larvae undergoing a successful molt, survival and diet contamination. The contribution of 2 lipid components (wheat germ oil and linseed oil) was tested in a separate experiment to confirm results produced in the 7-dimensional study. A reduced 3-dimensional design (4 ingredients) was used to identify an optimal formulation of the 4 ingredients identified in the screening design (Lapointe et al. 2008). Optimization in this context refers to the best combination of the ingredients studied. The predicted optimal blend of 4 ingredients was then validated in a separate experiment in comparison with the Pleau et al. diet.

\section{Screening design}

The diet ingredients used in this study included 17 ingredients used in a previously published formulation for WCR larvae (Pleau et al. 2002) plus wheat germ oil (Table 1). The screening design was formulated to simultaneously vary 8 diet components: wheat germ, casein, corn root powder, cellulose, sucrose, linseed oil, wheat germ oil and agar. Other ingredients (vitamin and salt mixtures, preservatives and antibiotics) were kept constant at the level reported 
by Pleau et al. (2002). No attempt was made to deconvolute two commonly used insect diet supplements, Wesson salt mix and Vanderzant vitamin mix (Lapointe et al. 2008). A 7dimensional mixture screening design was created with Design-Expert (v.10.0, Stat-Ease, Inc., Minneapolis, MN) resulting in 22 design points including vertex, center, 7-blend, and axial check blend points (Table 2) (Anderson and Whitcomb 2004). The resulting simplex, linear design had 7 model, 9 lack of fit and 5 pure error degrees of freedom (Myers and Montgomery 2002). Duplication of design points was sufficient to estimate pure error across the design space and to attain near uniform leverage for all design points (Weisberg 1985).

\section{4-component mixture design}

The 4 ingredients responsible for the greatest improvement in WCR larval weight in the screening design (not including lipid components) were used to construct an I-optimal mixture design sufficient to satisfy a Scheffé special cubic polynomial response surface model (Table 3) (Cornell 2002). The design consisted of 21 design points with 13 model, 2 lack of fit and 5 pure error degrees of freedom. A set of equations was derived that described insect responses (i.e., survival, molt from $1^{\text {st }}$ to $2^{\text {nd }}$ instar, and weight) at set time points (i.e., 7 day or 11 day) to varying blends of 4 key ingredients (wheat germ, casein, corn root powder and cellulose). Optimal blends were predicted using a simplex hill-climbing algorithm included in Design-Expert ${ }^{\mathrm{TM}}$ software (Lapointe et al. 2008). The equations enabled us to calculate the best optimal formulation based on desirable criteria (e.g. molt, weight, survival, contamination). 


\section{2-component mixture design for lipid components}

The 2 lipid ingredients, wheat germ oil and linseed oil, were used to construct an Ioptimal mixture design consisting of 5 design points including center, vertex and axial check blend points (Table 4). Response surface models were generated to validate the results produced in the screening design and to illustrate the contribution of lipid components of the Pleau et al. diet.

\section{Diet optimization}

A formulation for maximizing larval performance (survival, molting, and weight) was calculated with Design-Expert $^{\mathrm{TM}}$. This software used direct search methods (Derringer 1980) to maximize the desirability function (Lapointe et al. 2008, Myers et al. 2016).

\section{Model evaluation}

All three measures of larval performance (survival, molting, and weight) reared on the improved diet identified by the response surface mixture model were compared with those of larvae reared on the published WCR diet (Pleau et al. 2002).

\section{Statistical analyses}

Survival and molting data were generated by dividing the numbers of live larvae and successful larval molts from $1^{\text {st }}$ to $2^{\text {nd }}$ instar per replicate, respectively, by the initial number of larvae infested and multiplying by 100 to obtain percentages. Weight data were generated by dividing total weight per replicate by the number of live larvae.

For each measured response of larval performance (larval survival, proportion of successful larval molts and larval weight), all possible models from the mean to quartic 
polynomial were calculated with Design Expert ${ }^{\circledR}$. Initial model selection was based on criteria that included a lack of aliased terms, low residual values, low model $P$-value, nonsignificant lack

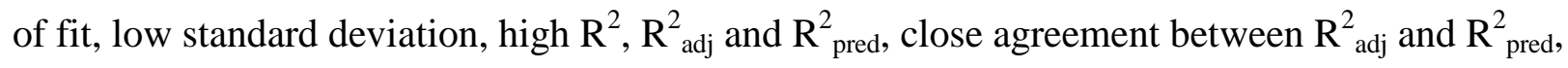
and a low PRESS value. $\mathrm{R}^{2}$ is a measure of variation around the mean explained by the response surface model. $\mathrm{R}^{2}$ can become biased if extraneous model terms are introduced. Therefore, the adjusted- $\mathrm{R}^{2}\left(\mathrm{R}_{\text {adj }}^{2}\right)$ decreases as the number of terms in the model increases if those additional terms do not increase the precision of the model (Lapointe et al. 2008). PRESS is the prediction error sum of squares (Allen 1971) calculated by removing a single observation from the response surface model, predicting that response point with the remaining $n-1$ observations, repeating this process for all observations, and then summing the squares of the $n$ PRESS residuals (Myers and Montgomery 2002). If two or more models were satisfactory, then the most stringent one was chosen. The selected model was then further evaluated according to a battery of adequacy tests as described by Anderson and Whitcomb (Anderson and Whitcomb 2004, Anderson and Whitcomb 2007) and Lapointe et al. (2008).

In the validation experiment, measured parameters of larval performance on the Pleau et al. diet and an improved diet at 7 and 11 days after infestation were analyzed as a randomized complete block design using PROC MIXED in SAS (SAS 2013). All percent variables were arcsine square-root transformed prior to the analysis to meet assumptions of normality and homoscedasticity. 


\section{Results}

\section{8-component screening experiment}

The screening design produced a significant linear response model for larval weight $(p=0.009, F=4.44$, d.f. $=7)$ by varying 8 diet components: agar, sucrose, wheat germ, casein, cellulose, corn root powder, wheat germ oil and linseed oil (Table 1, Table 2). We found a direct positive relationship between weight gain and proportion of casein, wheat germ, corn root and linseed oil, shown as a Piepel trace plot (Fig 1). Piepel trace plots estimate the effect of increasing the proportion of one component in relation to a reference blend while the relative proportions of all of the other diet components are kept constant (Smith 2005). In Figure 1, a linear model resulted in linear traces. The slope of the line indicates the direction and magnitude of the influence of the individual factors on the measured response variable, e.g., larval weight.

The greatest increase in weight was seen with proportional increases of casein and wheat germ. In contrast, we found that weight decreased with increasing proportions of agar, cellulose, sucrose and wheat germ oil (Fig 1). The greatest decrease in weight was seen when the proportion of agar was increased. Given that six ingredients (i.e., wheat germ, casein, corn root, linseed oil, wheat germ oil and sucrose) had the greatest influence on larval weight, they were chosen for further experimentations. Wheat germ, casein, corn root and sucrose were used within a 4-component mixture design and wheat germ oil and linseed oil were used within a 2component mixture design.

\section{4-component mixture experiment}

The 4-component mixture design yielded significant response surface models for all three measures of larval performance (survival, molt, and weight) by varying 4 diet components: 
wheat germ, casein, corn root powder and sucrose (Table 3), shown as Ternary plots (Fig 2). The ternary plots represent the universe of possible combinations of proportions of three components while proportions of other components (if applicable) are constant. In this case, prior knowledge constrained the amounts of certain diet ingredients resulting in the constrained regions shown in the response surfaces in Figs. 2A-C. The labelled isobars and color indicate the magnitude of the response variables in a dimension that can be envisioned as perpendicular to the page.

The mixture design produced a significant special cubic model for weight $(p<0.001, F$ $=128.74$, d.f. $=10$ ). The model was improved by stepwise regression (reduced). $R^{2}, R^{2}$ adj and $\mathrm{R}_{\text {pred, }}^{2}(0.99,0.99$ and 0.95 , respectively) were clustered with a difference $<0.05$. Lack-of-fit was not significant (Table 5). The response surface for weight gain indicated that casein was the most important component for maximizing larval weight. Wheat germ and corn root powder had a positive influence on weight while sucrose had a negative effect (Fig 2A). Interestingly, casein and corn root powder had an inverse blending effect (interaction), in which increasing proportion of casein with decreasing proportion of corn root powder resulted in an increase in weight (Fig 3A).

The best-fit model for survival was quadratic $(p<0.001, F=8.77$, d.f. $=4)$. The model was improved by stepwise regression. $R^{2}, R^{2}$ adj and $R^{2}$ pred $(0.69,0.61$ and 0.45 , respectively) were clustered and lack of fit was not significant (Table 5). Wheat germ and corn root powder were both influential in maximizing the number of larvae that survived at 7 days post infestation. In contrast, casein and sucrose at higher proportions had negative effects on larval survival rates (Fig 2B).

The mixture design produced a significant special cubic model for the percent of larvae that successfully completed a molt $(p<0.001, F=33.16$. d.f. $=11)$. Percent molting was 
transformed by arcsine square root. The model was improved by stepwise regression. $\mathrm{R}^{2}, \mathrm{R}^{2}$ adj and $\mathrm{R}_{\text {pred }}^{2}$ were $0.98,0.95$ and 0.90 , respectively. Lack of fit was not significant (Table 5). Corn root powder had a very strong influence on the developmental rate (measured by time to molt). The percentage of larval molts increased with increasing proportion of corn root powder. Additionally, increased casein, wheat germ, and sucrose lowered molting rates (Fig 2C). Results also showed very strong antagonistic blending effects of casein $\mathrm{x}$ sucrose and casein $\mathrm{x}$ wheat germ, indicating that an increase in casein at higher proportions with decreasing proportions of sucrose or wheat germ resulted in decreased molting (Fig 3B \& 3C).

There was an inverse relationship between weight, survival and molting when proportions of corn root powder, casein and wheat germ varied; shown as Piepel trace plots (Fig 4). The quadratic models describing the responses to the four-blend diet showed curvature and revealed nonlinear relationships between proportionality of some diet components and the response variable. Specifically, an increase in corn root powder up to a proportion of $2.7 \%$ (w/w) resulted in an increase in both molting and survival, but an increase in corn root powder higher than the proportion of $1.2 \%(\mathrm{w} / \mathrm{w})$ resulted in a decrease in weight. However, high proportion of casein had a positive effect on larval weight gain, but a negative effect both on molting and survival. An increase in casein up to the proportion of $3.8 \%(\mathrm{w} / \mathrm{w})$ maximized larval weight whereas a decrease in casein below $1.8 \%(\mathrm{w} / \mathrm{w})$ caused a decrease in molting. An increase in proportion of wheat germ up to the level of $6.3 \%(\mathrm{w} / \mathrm{w})$ resulted in an increase in survival but higher proportions resulted in a decrease in weight and molting. In contrast, an increase in wheat germ above $3.5 \%(\mathrm{w} / \mathrm{w})$ and $4.6 \%(\mathrm{w} / \mathrm{w})$ resulted in a decrease in weight and molting, respectively. Consequently, the optimum proportion of corn root powder, casein and wheat germ for molting, survival and weight were different. 


\section{2-component lipid mixture experiment}

Response surface models for weight and survival were generated by varying 2 lipid components: wheat germ oil and linseed oil (Table 5). Models indicated that the 2 lipid components had a minor contribution on improving larval weight, survival and molting.

The linear model for survival was significant $(p=0.049)$. The model indicated that linseed oil had a positive effect while wheat germ oil had a slightly negative effect on larval survival (Fig 5). The best model for weight gain was the overall mean. Neither oil had an effect on weight.

The model for molting was not generated because few larvae molted to second instar during this experiment. Within the range of proportions tested, the 2 oil components did not improve molting from first to second instar.

\section{Contamination}

There was minor contamination $(<1 \%)$ during all experiments and all time points evaluated. Consequently, there was no evidence for a relationship between contamination and diet ingredients.

\section{Model evaluation and diet improvement}

The diet optimization for WCR larvae produced a formulation, referred to hereafter as WCRMO-1 (Table 6), that yielded better larval performance compared with the Pleau et al. diet (Table 7). WCRMO-1 was formulated in terms of maximizing an overall value of all three diet traits (survival, molt, and weight) simultaneously by optimizing the concentration of ingredients in the first WCR diet described by Pleau et al. (2002). At 7 days post infestation, 
there was a significant difference in survival rate between larvae reared on WCRMO-1 and on the Pleau et al. diet, but there were no significant differences in larval dry weight and molting rate. However, a significant improvement was seen in all three measures of larval performance at 11 days post infestation. At 11 days after infestation, the three measures (survival, molt, and weight) were significantly higher for larvae reared on WCRMO-1 compared with larvae reared on the Pleau et al. diet (Table 7). The level of improvement in larval dry weight gain was a twofold increase and the survival and molting rate for larvae reared on WCRMO-1 were approximately $99 \%$, compared to $93 \%$ and $90 \%$, respectively when reared on the Pleau et al. diet.

\section{Discussion}

The application of RSM based on a multivariate mixture design enabled us to concurrently evaluate several ingredients of an artificial diet for rearing WCR. By applying this method to a published formulation (Pleau et al. 2002), we optimized 8 ingredients based on life history criteria (survival, molt, and weight). By applying good laboratory practices, as described above, we were able to decrease contamination and extend the assay period to 11 days and thereby measure events that had not occurred within shorter assay periods, e.g., molting and survival.

Results indicated that corn root powder, casein and wheat germ were key ingredients effecting larval performance, i.e., changing the concentration of these ingredients affected the life history parameters. In contrast, changing the proportion of other ingredients, including cellulose, sucrose, linseed oil, wheat germ oil and agar, had little or no negative effects on the response parameters. 
We observed complex relationships between the response parameters and the proportionality of the key ingredients. Relatively small adjustments in the proportions of these nutrients resulted in a dramatic improvement in diet performance. As has been reported for other insect diets (Lapointe et al. 2010a), we found an inverse relationship between weight, survival and molting as the proportions of corn root powder, casein, and wheat germ varied.

Corn root powder provided the most pronounced example of these complex relationships. Our results showed that corn root powder at high proportions improved molting and survival, but caused a decrease in weight when changed in combination with casein, wheat germ and sucrose. Pleau et al. (2002) reported a similar pattern in which corn root powder at high proportion did not yield larval weight gain with an OIAT design. An increase in proportion of corn root powder up to $0.6 \%(\mathrm{w} / \mathrm{v})$ resulted in an increase in larval weight, but corn root powder at higher concentrations did not yield an increase in weight (Pleau et al. 2002).

Our results indicated that casein also had a complex relationship with the life history parameters. Pleau et al. (2002) documented that casein in the diet contributed significantly to WCR larval weight gain with an OIAT design. Cohen (2015) suggested that an inclusion of the casein in diet mixtures may result in important interactions with other ingredients. Casein can reduce gel strength, possibly by reducing the capability of agar to bind water (Cohen 2015), personal observation) or by binding calcium ions which may debilitate cross-linking in the gel matrix (Swaisgood 2008) .

We also found a complex relationship between wheat germ and life history parameters. There was a direct correlation between the proportion of wheat germ and survival but at high proportions there was a negative effect on larval weight and molting. Pleau et al. (2002) reported that an increase in wheat germ above $5.45 \%(w / v)$ had a negative effect on 
larval weight, but removal of wheat germ resulted in a significantly negative effect on WCR growth. Wheat germ is a key dietary component contributing to nutritional value, digestibility, bioavailability, and palatability, but at high concentrations can result in a deleterious effect on insect growth (Cohen 2015).

Contamination has been one of the limiting factors with diet when used to rear WCR and for toxicity-bioassays (Pereira et al. 2016). Previously, microbial contamination (bacteria and fungi) forced diet bioassays to be terminated early and prevented more extensive observations. Typically, diet bioassays for rearing WCR ran for 6 days (Pleau et al. 2002) while diet toxicity-bioassays ran for 4 to 7 days (Siegfried et al. 2005). Those time points preceded $1^{\text {st }}$ to $2^{\text {nd }}$ instar molting. We were able to eliminate contamination through good laboratory practices which allowed for longer bioassays. By extending the bioassay to 11 days of feeding, we were able to detect differences among the formulations that were not detected at 7 days of feeding. For example, at 7 days WCRMO-1 and the Pleau et al. diet had similar larval performance. However, at 11 days larval performance on WCRMO-1 was better than the Pleau et al. diet (Table 7). At 11 days post infestation, larval dry weight, survival and molting rate of WCR larvae reared on WCRMO-1 were significantly higher than that of the Pleau et al. diet. Larval dry weight increased two-fold with WCRMO-1 as compared to the published diet. Survival and molting increased to approximately 99\% with WCRMO-1.

WCR larval feeding typically ceases some hours before molting, and larval weight typically declines immediately following a molt ((Waldbauer 1968), personal observation). The improved performance in terms of weight gain of larvae reared on WCRMO-1 was particularly impressive in light of the greater proportion of those larvae that successfully completed the 
process of molting to the next instar compared with larvae reared on the Pleau et al. diet (Pleau et al. 2002).

The goal of an artificial diet should always be to produce insects that develop similarly to those reared on their natural food source (Lapointe et al. 2010b). WCR larvae molted at 7 days post infestation on maize (Wilson and Hibbard 2004). However, only $12.5 \%$ WCR larvae molted to second instar at 7 days when reared on WCRMO-1. While this is a major improvement over previous diets, and provides a diet suitable for toxicity bioassays (unpublished data, Hibbard/Coudron labs), these accomplishments also suggest additional formulation refinement may be possible and that a suitable substitution for corn root powder, which is not available from a commercial source, would be highly advantageous. 
Table 1. Components that were proportionally varied or held constant in diets used to rear western corn rootworm larvae.

\begin{tabular}{ll}
\hline \multicolumn{1}{c}{ Variable components } & \multicolumn{1}{c}{ Constant components } \\
\hline 1. Agar & 1. Chlortetracycline $(10 \mathrm{mg} / \mathrm{ml})$ \\
2. Casein & 2. Distilled water \\
3. Cellulose & 3. Cholesterol \\
4. Corn root powder & 4. Food coloring \\
5. Linseed oil & 5. Methyl paraben \\
6. Sucrose & 6. Potassium hydroxide $(10 \%)$ \\
7. Wheat germ, ground & 7. Sorbic acid \\
8. Wheat germ oil & 8. Streptomycin $(12.8 \mathrm{mg} / \mathrm{ml})$ \\
& 9. Vanderzant vitamin mix \\
& 10. Wesson's salt mix \\
\hline
\end{tabular}


Table 2. Diet blends of 8 components varied in a 7-dimensional, mixture screening design to rear western corn rootworm larvae

\begin{tabular}{rrrrrrrrr}
\hline Diet blend \# & $\begin{array}{c}\text { Agar } \\
(\mathrm{g})\end{array}$ & $\begin{array}{r}\text { Sucrose } \\
(\mathrm{g})\end{array}$ & $\begin{array}{r}\text { Wheat germ } \\
(\mathrm{g})\end{array}$ & $\begin{array}{c}\text { Casein } \\
(\mathrm{g})\end{array}$ & $\begin{array}{c}\text { Cellulose } \\
(\mathrm{g})\end{array}$ & $\begin{array}{c}\text { Corn root } \\
(\mathrm{g})\end{array}$ & $\begin{array}{c}\text { Linseed oil } \\
(\mu \mathrm{l})\end{array}$ & $\begin{array}{c}\text { Wheat germ oil } \\
(\mu \mathrm{l})\end{array}$ \\
\hline 1 & 1 & 1 & 7.02 & 5 & 1 & 1 & 0 & 0.05 \\
2 & 1 & 1 & 3.97 & 5 & 5 & 0 & 0.05 & 0.05 \\
3 & 2.89 & 2.89 & 3.97 & 2.89 & 2.89 & 0.50 & 0.02 & 0.025 \\
4 & 1 & 5 & 4.02 & 1 & 5 & 0 & 0.05 & 0 \\
5 & 5 & 1 & 8.02 & 1 & 1 & 0 & 0.05 & 0 \\
6 & 1 & 1 & 10 & 1 & 3.02 & 0 & 0 & 0.05 \\
7 & 5 & 5 & 1 & 3.02 & 1 & 1 & 0 & 0.05 \\
8 & 1 & 1 & 7.07 & 5 & 1 & 1 & 0 & 0 \\
9 & 1 & 1 & 8.02 & 5 & 1 & 0 & 0 & 0.05 \\
10 & 5 & 5 & 1 & 1 & 4.02 & 0 & 0 & 0.05 \\
11 & 2.89 & 2.89 & 3.97 & 2.89 & 2.89 & 0.50 & 0.025 & 0.025 \\
12 & 2.89 & 2.89 & 3.97 & 2.89 & 2.89 & 0.50 & 0.025 & 0.025 \\
13 & 5 & 4.02 & 1 & 5 & 1 & 0 & 0.05 & 0 \\
14 & 1 & 1 & 10 & 1 & 2.02 & 1 & 0 & 0.05 \\
15 & 3.01 & 1 & 10 & 1.05727 & 1 & 0 & 0 & 0 \\
16 & 2.89 & 2.89 & 3.97 & 2.89 & 2.89 & 0.50 & 0.025 & 0.025 \\
17 & 1 & 5 & 1 & 3.07 & 5 & 1 & 0 & 0 \\
18 & 2.89 & 2.89 & 3.97 & 2.89 & 2.89 & 0.50 & 0.025 & 0.025 \\
19 & 5 & 1 & 3.02 & 1 & 5 & 1 & 0.05 & 0 \\
20 & 5 & 1 & 1 & 2.97 & 5 & 1 & 0.05 & 0.05 \\
21 & 1 & 5 & 6.97 & 1 & 1 & 1 & 0.05 & 0.05 \\
22 & 1 & 5 & 6.97 & 1 & 1 & 1 & 0.05 & 0.05 \\
\hline
\end{tabular}


Table 3. Diet blends of 4 ingredients varied in a three-dimensional mixture design to rear western corn rootworm larvae

\begin{tabular}{crrrr}
\hline Diet blend \# & Sucrose $(\mathrm{g})$ & Wheat germ $(\mathrm{g})$ & Casein $(\mathrm{g})$ & Corn root powder $(\mathrm{g})$ \\
\hline 1 & 5 & 4 & 0 & 3 \\
2 & 5 & 1.2 & 2.8 & 3 \\
3 & 0 & 8.8 & 0.2 & 3 \\
4 & 0 & 5.3 & 5 & 1.7 \\
5 & 3.5 & 2.9 & 3.9 & 1.7 \\
6 & 1.2 & 10 & 0 & 0.8 \\
7 & 2.4 & 4.6 & 5 & 0 \\
8 & 0 & 10 & 2 & 0 \\
9 & 2.9 & 5 & 2.4 & 1.7 \\
10 & 0 & 8.8 & 0.2 & 3 \\
11 & 5 & 7 & 0 & 0 \\
12 & 0 & 5.3 & 5 & 0 \\
13 & 2.4 & 7.7 & 1.9 & 0 \\
14 & 2.4 & 4.6 & 5 & 3 \\
15 & 1 & 3.1 & 4.9 & 3 \\
16 & 5 & 4 & 0 & 0 \\
17 & 0 & 10 & 2 & 3 \\
18 & 3 & 1 & 5 & 0 \\
19 & 5 & 4.3 & 2.7 & 1 \\
20 & 5 & 1 & 5 & 1.5 \\
21 & 2.5 & 5.5 & 2.5 & \\
\hline
\end{tabular}


Table 4. Diet blends of 2 lipid components varied in a 1-dimensional mixture design to rear western corn rootworm larvae

\begin{tabular}{crr}
\hline Diet blend \# & Wheat germ oil $(\mu \mathrm{l})$ & Linseed oil $(\mu \mathrm{l})$ \\
\hline 1 & 50 & 450 \\
2 & 150 & 350 \\
3 & 250 & 250 \\
4 & 350 & 150 \\
5 & 450 & 50 \\
\hline
\end{tabular}


Table 5. $p$-values, regression coefficients and response surface model fitting diagnostic statistics for WCR larval responses to 4-component diet mixtures

\begin{tabular}{|c|c|c|c|c|c|c|}
\hline & $\begin{array}{l}\text { Weight } \\
p \text {-values }\end{array}$ & $\begin{array}{l}\text { Regression } \\
\text { coefficients }\end{array}$ & $\begin{array}{l}\% \text { Survival } \\
p \text {-values }\end{array}$ & $\begin{array}{l}\text { Regression } \\
\text { coefficients }\end{array}$ & $\begin{array}{l}\% \text { Molt } \\
p \text {-values }\end{array}$ & $\begin{array}{l}\text { Regression } \\
\text { coefficients }\end{array}$ \\
\hline Model & $<0.0001$ & - & 0.0006 & - & $<0.0001$ & - \\
\hline Linear mixture & $<0.0001$ & - & 0.0029 & - & $<0.0001$ & - \\
\hline A & - & 0.13 & - & 0.72 & - & -2.17 \\
\hline B & - & 0.05 & - & 0.97 & - & -0.33 \\
\hline $\mathrm{C}$ & - & -0.13 & - & 0.02 & - & -3.86 \\
\hline D & - & -0.67 & - & 0.65 & - & 1.28 \\
\hline $\mathrm{A} \times \mathrm{B}$ & 0.1981 & -1.88 & - & - & 0.0020 & 4.68 \\
\hline $\mathrm{A} \times \mathrm{C}$ & 0.1999 & 0.16 & - & - & $<0.0001$ & 12.61 \\
\hline$A \times D$ & 0.0014 & 0.76 & - & - & 0.7000 & -0.8 \\
\hline $\mathrm{B} \times \mathrm{C}$ & 0.0029 & 0.48 & - & - & $<0.0001$ & 8.12 \\
\hline$B \times D$ & 0.0005 & 0.85 & - & - & 0.5689 & -0.94 \\
\hline$C \times D$ & $<0.0001$ & 1.78 & 0.0020 & 4.19 & 0.0021 & 6.74 \\
\hline$A \times B \times C$ & 0.0105 & 1.42 & - & - & 0.0097 & 10.96 \\
\hline$A \times B \times D$ & - & - & - & - & 0.0052 & 9.47 \\
\hline Lack of fit & 0.3901 & & 0.6193 & & 0.9866 & \\
\hline Model type & $\begin{array}{l}\text { Special } \\
\text { Cubic } \\
\text { (reduced) }\end{array}$ & & $\begin{array}{l}\text { Quadratic } \\
\text { (reduced) }\end{array}$ & & $\begin{array}{l}\text { Special } \\
\text { Cubic } \\
\text { (reduced) }\end{array}$ & \\
\hline Transformation & N/A & & N/A & & $\begin{array}{l}\text { Arcsin Sqrt } \\
(\% \text { molt }+0.01)\end{array}$ & \\
\hline $\mathrm{R}^{2}$ & 0.9923 & & 0.6869 & & 0.9759 & \\
\hline $\mathrm{R}_{\text {adj }}^{2}$ & 0.9846 & & 0.6086 & & 0.9465 & \\
\hline $\mathrm{R}_{\text {pred }}^{2}$ & 0.9488 & & 0.4464 & & 0.8996 & \\
\hline
\end{tabular}

A: sucrose, B: wheat germ, C: casein, D: corn root powder. Arcsin Sqrt: arcsine square root. 
Table 6. Diet formulation improvement for WCR development (109 g).

\begin{tabular}{lcc} 
Ingredients & Pleau et al. (2002) & WCRMO-1 \\
\hline Sucrose & $3.85 \mathrm{~g}$ & $2.5 \mathrm{~g}$ \\
Wheat germ (raw, ground) & $5.45 \mathrm{~g}$ & $5.5 \mathrm{~g}$ \\
Casein & $3.23 \mathrm{~g}$ & $2.5 \mathrm{~g}$ \\
Cellulose & $1.38 \mathrm{~g}$ & $1.5 \mathrm{~g}$ \\
Corn root powder & $0.63 \mathrm{~g}$ & $1.5 \mathrm{~g}$ \\
Agar & $1.45 \mathrm{~g}$ & $1.5 \mathrm{~g}$ \\
Linseed oil, raw & $40 \mu \mathrm{l}$ & $25 \mu \mathrm{l}$ \\
Wheat germ oil & - & $25 \mu \mathrm{l}$ \\
Cholesterol & $6 \mathrm{mg}$ & $6 \mathrm{mg}$ \\
Wesson's salt mix & $0.93 \mathrm{~g}$ & $0.93 \mathrm{~g}$ \\
Vanderzant Vitamin mix & $0.90 \mathrm{~g}$ & $0.90 \mathrm{~g}$ \\
Methyl paraben & $0.10 \mathrm{~g}$ & $0.10 \mathrm{~g}$ \\
Sorbic acid & $64 \mathrm{mg}$ & $64 \mathrm{mg}$ \\
Potassium hydroxide $(10 \%)$ & $3.5 \mathrm{ml}$ & $3.5 \mathrm{ml}$ \\
Streptomycin $(12.8 \mathrm{mg} / \mathrm{ml})$ & $6.4 \mathrm{mg}$ & $6.4 \mathrm{mg}$ \\
Chlortetracycline $(10.0 \mathrm{mg} / \mathrm{ml})$ & $6.4 \mathrm{mg}$ & $6.4 \mathrm{mg}$ \\
Distilled water & $88 \mathrm{ml}$ & $88 \mathrm{ml}$ \\
Green food coloring & $64 \mu \mathrm{l}$ & $64 \mu \mathrm{l}$ \\
\hline
\end{tabular}


Table 7. Larval dry weight, survival, and percent successful completion of molt for western corn rootworm larvae reared on WCRMO-1 and Pleau et al. (2002) diets for 7 or 11 days.

\begin{tabular}{lccccccc} 
& \multicolumn{3}{c}{7 days } & & \multicolumn{3}{c}{11 days } \\
\cline { 2 - 4 } \cline { 6 - 8 } Diet & $\begin{array}{c}\text { Weight } \\
(\mu \mathrm{g})\end{array}$ & $\begin{array}{c}\text { Survival } \\
(\%)\end{array}$ & $\begin{array}{c}\text { Molt } \\
(\%)\end{array}$ & & $\begin{array}{c}\text { Weight } \\
(\mu \mathrm{g})\end{array}$ & $\begin{array}{c}\text { Survival } \\
(\%)\end{array}$ & $\begin{array}{c}\text { Molt } \\
(\%)\end{array}$ \\
\hline WCRMO-1 & $100 \pm 3.1$ & $99 \pm 2.1 \mathrm{a}$ & $13 \pm 5.9$ & & $425 \pm 54.7 \mathrm{a}$ & $99 \pm 1.8 \mathrm{a}$ & $99 \pm 1.8 \mathrm{a}$ \\
Pleau et al. & $106 \pm 16.1$ & $91 \pm 5.2 \mathrm{~b}$ & $4 \pm 3.4$ & & $196 \pm 32.4 \mathrm{~b}$ & $93 \pm 4.7 \mathrm{~b}$ & $90 \pm 4.8 \mathrm{~b}$ \\
\hline
\end{tabular}

Means within columns followed by different letters are significantly different $(\alpha=0.05$, ANOVA). Mean \pm SD. 


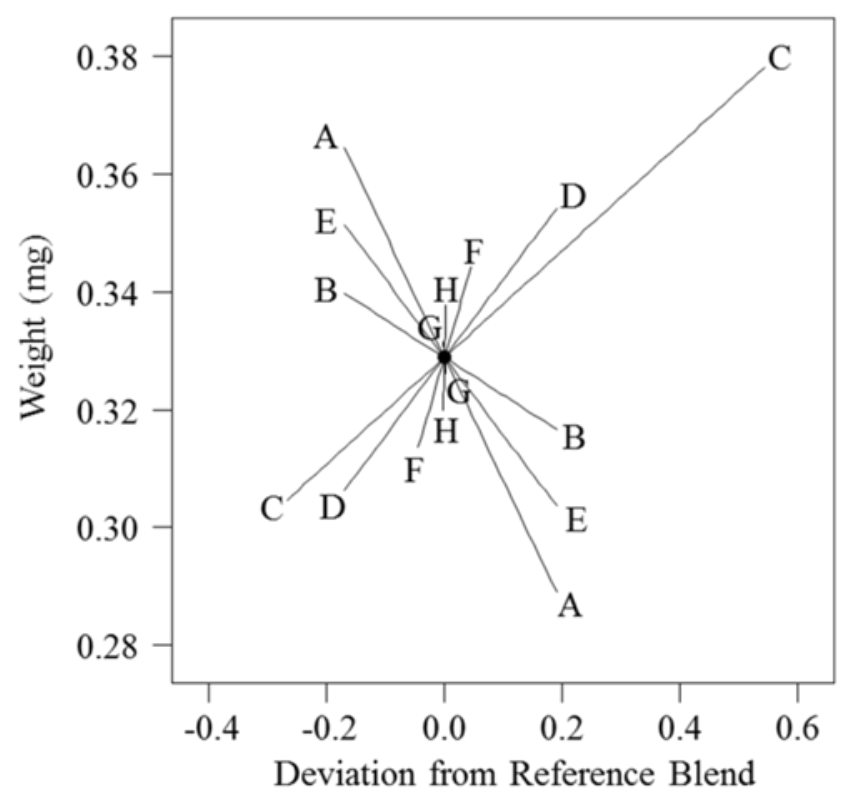

Figure 1. Piepel trace plot of larval weight deviation from a reference blend diet. Reference blend proportions: agar $=0.171$, sucrose $=0.171$, wheat germ $=0.267$, casein $=0.171$, cellulose $=$ 0.171, corn root: 0.045 , wheat germ oil $=0.002$, linseed oil $=0.002$. A: agar, B: sucrose, C: wheat germ, D: casein, E: cellulose, F: corn root, G: wheat germ oil, H: linseed oil. 

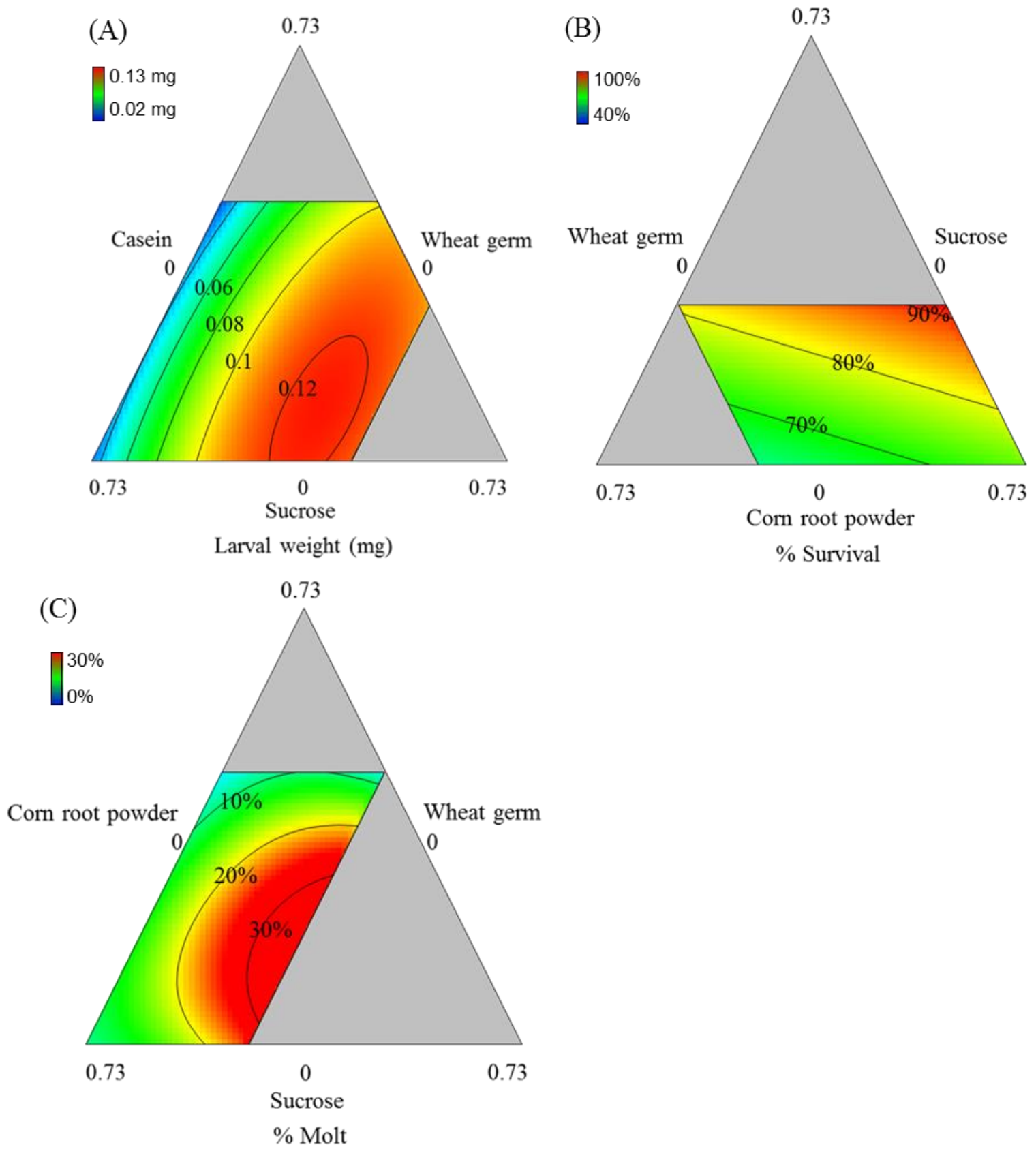

Figure 2. Predicted 3-D surface response plots for WCR larvae reared on different diets. (A) weight, (B) survival, and (C) molt, at 7 days post infestation. For (A) the proportion of corn root powder was constant at 0.27 . For (B) and (C) the proportion of casein was constant at 0.27 . Color bars display the magnitude of the measured response. 

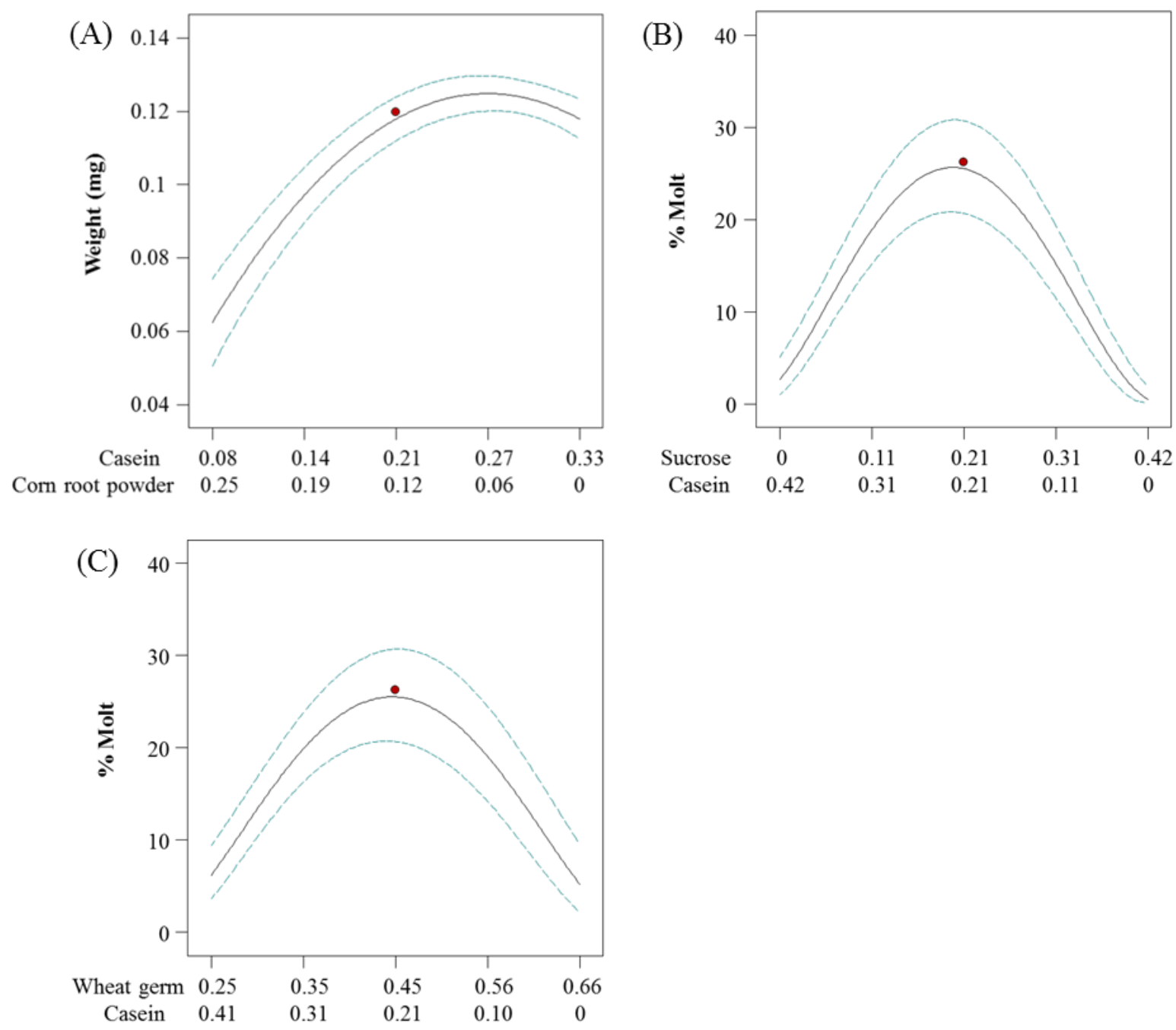

Figure 3. Nonlinear blending effects in the 4-component mixture experiment. (A) casein $\mathrm{x}$ corn root powder, (B) casein x sucrose, and (C) casein x wheat germ. Dotted lines indicate 95\% confidence interval bands. 


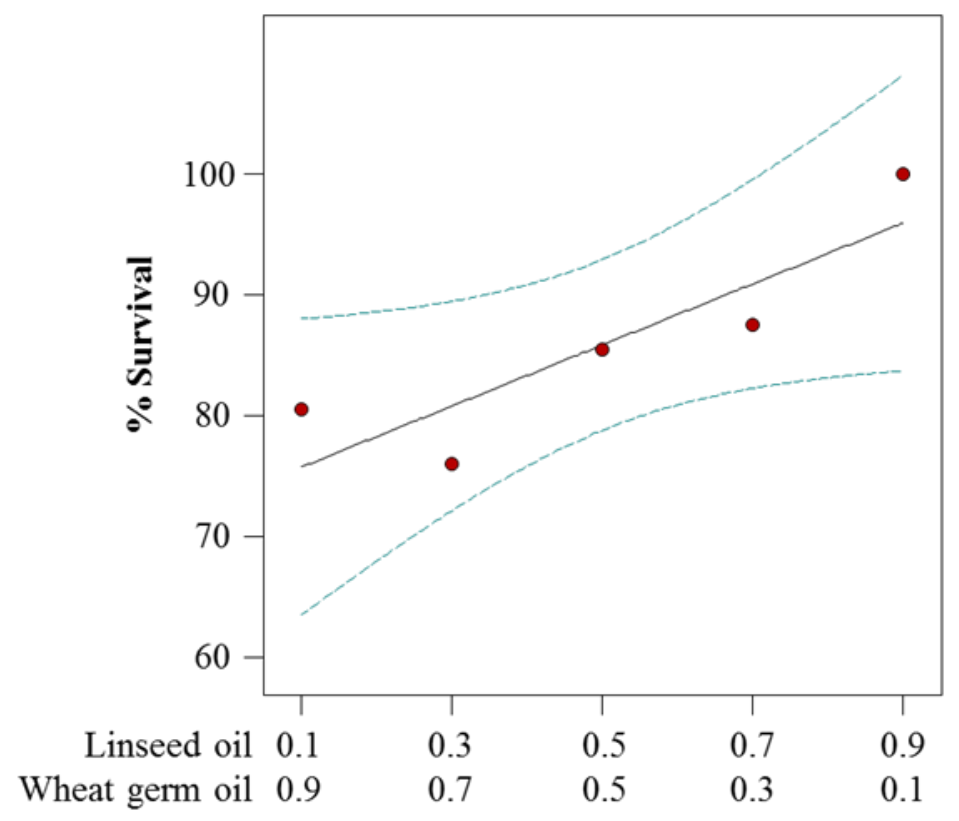

Figure 4. Predicted survival plots for WCR larvae reared on diets that vary in lipid content. Red dots are predicted values by the model at 7 days post infestation. Dotted lines indicate $95 \%$ confidence interval bands. Regression equation: Survival $=0.99 \times$ Linseed oil $+0.73 \times$ Wheat germ oil. 

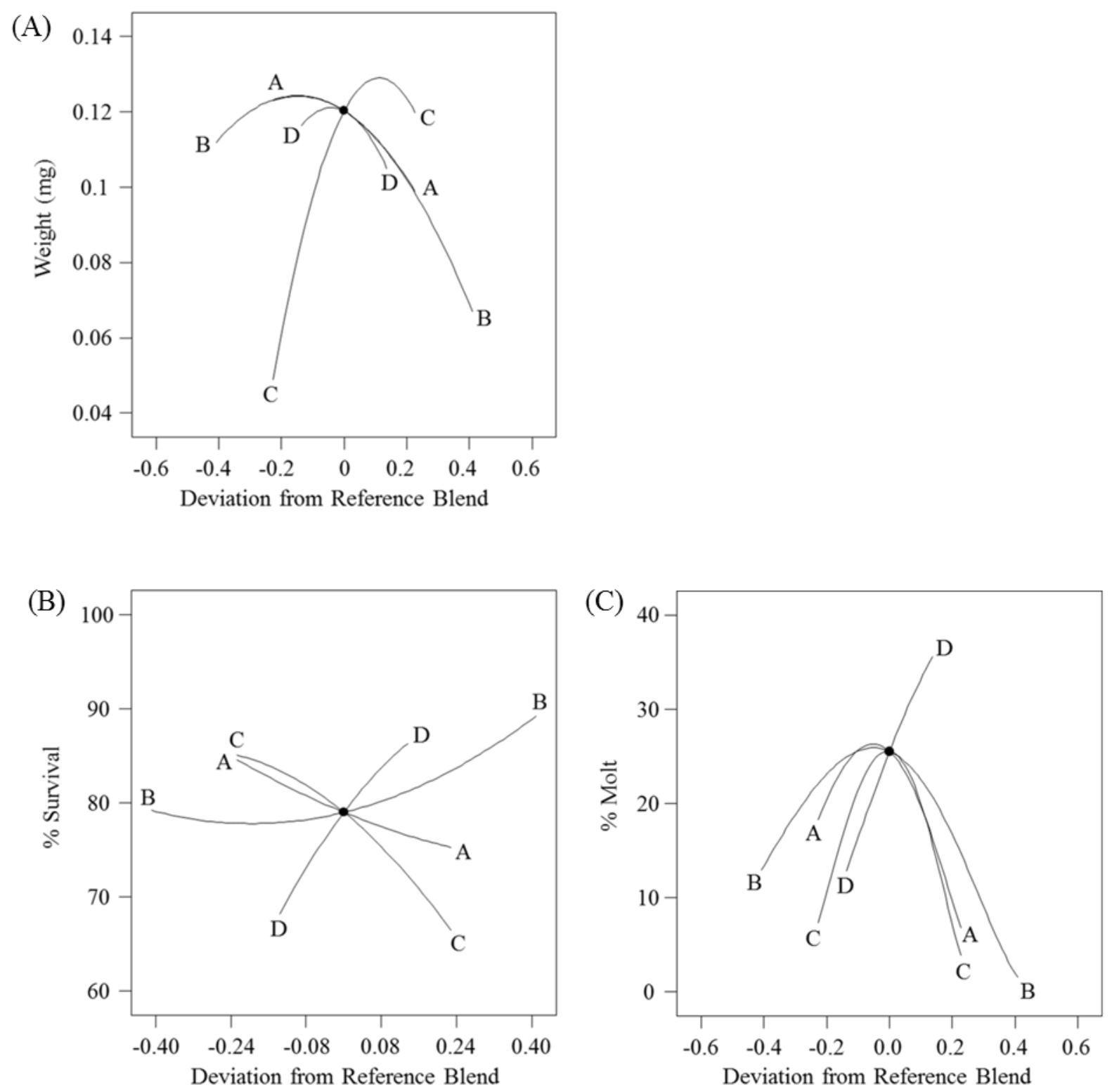

Figure 5. Piepel trace plots of weight, survival and molt deviation from a reference diet. (A) weight, (B) survival, and (C) molt. Reference blend proportions: sucrose $=0.227$, wheat germ $=$ 0.409 , casein $=0.227$, corn root powder $=0.136$. Within each figure: A: Sucrose, B: wheat germ, C: casein, and D: corn root. 


\section{References}

Allen, D. M. 1971. Mean square error of prediction as a criterion for selecting variables. Technometrics 13: 469-475.

Anderson, M. J., and P. J. Whitcomb. 2004. RSM simplified: optimizing processes using response surface methods for design of experiments. CRC press.

Anderson, M. J., and P. J. Whitcomb. 2007. Using graphical diagnostics to deal with bad data. Qual. Eng. 19: 111-118.

Ball, H. J., and G. T. Weekman. 1963. Differential resistance of corn rootworms to insecticides in Nehraska and adjoining States. J. Econ. Entomol. 56: 553-555.

Cohen, A. C. 2015. Insect diets: science and technology, 2nd ed. Taylor \& Francis Group.

Cornell, J. A. 2002. Experiments with mixtures: designs, models, and the analysis of mixture data, 3rd ed. John Wiley \& Sons, Inc., New York, USA.

Culy, M. D., C. R. Edwards, and J. R. Cornelius. 1992. Effect of silk feeding by western corn rootworm (Coleoptera: Chrysomelidae) on yield and quality of inbred corn in seed corn production fields. J. Econ. Entomol. 85: 2440-2446.

Derringer, G. 1980. Simultaneous optimization of several response variables. J. Qual. Technol. 12: $214-219$.

EPA. 2014. EPA Proposal to improve corn rootworm resistance management. In U. S. E. P. Agency [ed.]. United States Environmental Protection Agency, Washington, DC, USA.

EPA. 2016a. EPA docket for corn rootworm resistance management and framework for Bt corn.

In U. S. E. P. Agency [ed.]. United States Environmental Protection Agency, Washington, DC, USA.

EPA. 2016b. EPA needs better data, plans and tools to manage insect resistance to geneticlly engineered corn. In U. S. E. P. Agency [ed.]. United States Environmental Protection Agency, Washington, DC, USA.

Gassmann, A. J., J. L. Petzold-Maxwell, R. S. Keweshan, and M. W. Dunbar. 2011. Fieldevolved resistance to Bt maize by western corn rootworm. PloS one 6: e22629. 
Gassmann, A. J., R. B. Shrestha, S. R. Jakka, M. W. Dunbar, E. H. Clifton, A. R. Paolino, D. A. Ingber, B. W. French, K. E. Masloski, and J. W. Dounda. 2016. Evidence of resistance to Cry34/35Ab1 corn by western corn rootworm (Coleoptera: Chrysomelidae): Root injury in the field and larval survival in plant-based bioassays. J. Econ. Entomol. 109: 18721880.

Gray, M. E., T. W. Sappington, N. J. Miller, J. Moeser, and M. O. Bohn. 2009. Adaptation and invasiveness of western corn rootworm: intensifying research on a worsening pest. Ann. Rev. Entomol. 54: 303-321.

Lapointe, S. L., T. J. Evens, and R. P. Niedz. 2008. Insect diets as mixtures: Optimization for a polyphagous weevil. J. Ins. Physiol. 54: 1157-1167.

Lapointe, S. L., R. P. Niedz, and T. J. Evens. 2010a. An artificial diet for Diaprepes abbreviatus (Coleoptera: Curculionidae) optimized for larval survival. Fla. Entomol. 93: 56-62.

Lapointe, S. L., T. J. Evens, R. P. Niedz, and D. G. Hall. 2010b. Artificial diet optimized to produce normative adults of Diaprepes abbreviatus (Coleoptera: Curculionidae). Environ. Entomol. 39: 670-677.

Levine, E., J. L. Spencer, S. A. Isard, D. W. Onstad, and M. E. Gray. 2002. Adaptation of the western corn rootworm to crop rotation: evolution of a new strain in response to a management practice. Am. Entomol. 48: 94-117.

Ludwick, D., and B. Hibbard. 2016. Rootworm management: status of GM traits, insecticides, and potential new tools. CABI. 11:1-10.

Ludwick, D. C., L. N. Meihls, K. R. Ostlie, B. D. Potter, L. French, and B. E. Hibbard. 2017. Minnesota field population of western corn rootworm (Coleoptera: Chrysomelidae) shows incomplete resistance to Cry34Ab1/Cry35Ab1 and Cry3Bb1. J. Appl. Entomol. 141: $28-40$.

Marrone, P. G., F. D. Ferri, T. R. Mosley, and L. J. Meinke. 1985. Improvements in laboratory rearing of the southern corn rootworm, Diabrotica undecimpuncta howardi Barber (Coleoptera: Chrysomelidae), on an artificial diet and corn. J. Econ. Entomol. 78: 290293. 
Meinke, L. J., B. D. Siegfried, R. J. Wright, and L. D. Chandler. 1998. Adult susceptibility of Nebraska western corn rootworm (Coleoptera: Chrysomelidae) populations to selected insecticides. J. Econ. Entomol. 91: 594-600.

Mitchell, P. D. 2011. Costs and benefits of controlling pest Diabrotica in maize in the United States. In, 24th IWGO Conference and 3rd International Conference of Diabrotica Genetics, IWGO, Freiburg, Germany.

Myers, R. H., and D. C. Montgomery. 2002. Response surface methodology: process and product optimization using designed experiments, 2nd ed., Willey, New York, NY, USA.

Myers, R. H., D. C. Montgomery, and C. M. Anderson-Cook. 2016. Response surface methodology: process and product optimization using designed experiments, 4th ed., John Wiley \& Sons, New York, NY, USA.

Niedz, R. P., and T. J. Evens. 2016. Design of experiments (DOE)—history, concepts, and relevance to in vitro culture. In Vitro Cell. Dev. Biol. - Plant 52: 547-562.

Pascacio-Villafán, C., S. Lapointe, T. Williams, J. Sivinski, R. Niedz, and M. Aluja. 2014. Mixture-amount design and response surface modeling to assess the effects of flavonoids and phenolic acids on developmental performance of Anastrepha ludens. J. Chem. Ecol. 40: 297-306.

Pereira, A. E., N. P. Carneiro, and B. D. Siegfried. 2016. Comparative susceptibility of southern and western corn rootworm adults and larvae to vATPase-A and Snf7 dsRNAs. J. RNAi and Gene Silencing 12: 528-535.

Pereira, A. E., H. Wang, S. N. Zukoff, L. J. Meinke, B. W. French, and B. D. Siegfried. 2015. Evidence of field-evolved resistance to Bifenthrin in western corn rootworm (Diabrotica virgifera virgifera LeConte) populations in Western Nebraska and Kansas. PloS one 10: e0142299.

Pleau, M. J., J. E. Huesing, G. P. Head, and D. J. Feir. 2002. Development of an artificial diet for the western corn rootworm. Entomol. Exp. Appl. 105: 1-11.

Rose, R. I., and J. M. McCabe. 1973. Laboratory rearing techniques for the southern corn rootworm. J. Econ. Entomol. 66: 398-400. 
Ruohonen, K., and J. Kettunen. 2004. Effective experimental designs for optimizing fish feeds. Aquacult. Nutr. 10: 145-151.

SAS. 2013. SAS version 9.4, SAS Institute, Cary, N.C, USA.

Scheffé, H. 1958. Experiments with mixtures. Royal Stat. Soc., Series B (Methodological) 20: 344-360.

Siegfried, B. D., T. T. Vaughn, and T. Spencer. 2005. Baseline susceptibility of western corn rootworm (Coleoptera: Crysomelidae) to Cry3Bb1 Bacillus thuringiensis toxin. J. Econ. Entomol. 98: 1320-1324.

Smith, W. F. 2005. Experimental design for formulation. ASA-SIAM series on statistics and applied probability. SIAM, Philadelphia, PA, USA.

Sutter, G. R., J. L. Krysan, and P. L. Guss. 1971. Rearing the southern corn rootworm on artificial diet. J. Econ. Entomol. 64: 65-67.

Swaisgood, H. 2008. Characteristics of milk. In: S. Damodaran, K. L. Parkin and O. R. Fennema, Eds., Fennema’s Food Chemistry, vol. 169, CRC Press, Boca Raton, FL, USA.

Waldbauer, G. 1968. The consumption and utilization of food by insects. Adv. Insect Physiol. 5: 229-288.

Weisberg, S. 1985. Applied linear regression, 2nd ed., Wiley \& Sons, New York, NY, USA.

Wilson, T. A., and B. E. Hibbard. 2004. Host suitability of nonmaize agroecosystem grasses for the western corn rootworm (Coleoptera : Chrysomelidae). Environ. Entomol. 33: 11021108.

Zukoff, S. N., K. R. Ostlie, B. Potter, L. N. Meihls, A. L. Zukoff, L. French, M. R. Ellersieck, B. Wade French, and B. E. Hibbard. 2016. Multiple assays indicate varying levels of cross resistance in Cry3Bb1-selected field populations of the western corn rootworm to mCry3A, eCry3.1Ab, and Cry34/35Ab1. J. Econ. Entomol. 109:1387-1398. 


\title{
CHAPTER III: ISOLATION AND CHARACTERIZATION OF CORN ROOT FACTORS FOR IMPROVED GROWTH OF WESTERN CORN ROOTWORM LARVAE REARED ON ARTIFICIAL DIET
}

\begin{abstract}
The western corn rootworm is (WCR), Diabrotica virgifera virgifera LeConte, is an important economic pest of maize (Zea mays L.) in North America and Europe. Previous efforts to develop an artificial diet for WCR larvae highlighted an important role of corn root powder, which had a significant positive impact on several larval developmental traits. Unfortunately, this ingredient is not available to purchase. Toward a goal of formulating an open access diet for WCR larvae with all ingredients accessible, we documented that essential growth factors for larval development could be extracted from corn root powder to increase the nutrition of the diet. Whole corn root powder was best when used in combination with other nutritional sources in the published WCR formulation, but corn root powder alone without the addition of other macronutrient sources failed to support larval growth. In addition, we showed that corn root powder made from Monsanto proprietary seed and Viking seed have similar value and powder milled by both Monsanto (St. Louis, MO) and the Biological Control of Insect Research Laboratory (USDA-ARS, Columbia, MO) are similar. For all experiments, multiple life history parameters (survival, weight, and molting) were recorded from 15-day diet bioassays. This study demonstrated that corn roots contain factors that assist in larval growth and that most of these factors can be extracted, suggesting substituting corn root powder with compounds identified in the future from the extract may be possible.
\end{abstract}




\section{Introduction}

The western corn rootworm (WCR), Diabrotica virgifera virgifera LeConte, is an important insect pest of maize (Zea mays L.) in North America and Europe, causing more than $\$ 1$ billion in crop losses and control costs annually (Metcalf 1986, Gray et al. 2009). Rootworm larvae feeding on roots of maize are responsible for most of the damage associated to this species whereas adult WCR feeding on silks, pollen, kernels and foliage of maize plants (Branson and Krysan 1981, Moeser and Vidal 2005) may cause yield reduction if present at high densities before anthesis (Culy et al. 1992). Management of rootworm has been a challenge to industry and corn growers because the evolution of resistance to management tactics including chemical insecticides (Ball and Weekman 1963, Meinke et al. 1998, Pereira et al. 2015, Pereira et al. 2017), crop rotation (Levine et al. 2002) and transgenic maize hybrids expressing insecticidal crystalline toxins from Bacillus thuringiensis (Bt) (Gassmann et al. 2011, Zukoff et al. 2016, Ludwick et al. 2017).

Monitoring programs to detect differences in susceptibility attributable to resistance development of WCR populations are mandated by the U.S. Environmental Protection Agency (EPA) (EPA 2016). Diet-toxicity assays can be used as a tool for evaluating resistance. An artificial diet is a critical component of diet toxicity assay. An artificial diet capable of supporting larval growth and development that is similar to larvae reared on corn roots would be greatly beneficial for research programs and continuous rearing for WCR.

The first artificial diet for WCR larvae was developed by Pleau et al. (2002), which was a modification of a diet for southern corn rootworm (SCR), Diabrotica undecimpunctata howardi Barber. The SCR formulation with wheat germ and casein as the major nutritional components was a modification of a diet developed for a lepidopteran species, pink bollworm, 
Pectinophora gossypiella (Saunders) (Sutter et al. 1971, Rose and McCabe 1973, Marrone et al. 1985). The SCR is a generalist feeding on over 100 different plants while WCR and NCR are nearly monophagous on corn roots, but can survive on some grass species (Oyediran et al. 2004, Oyediran et al. 2008). Pleau et al. (2002) developed an initial formulation for WCR larvae by modifying the SCR formulation that included corn root powder, removing formalin, and altering concentrations of several additional ingredients (wheat germ, linseed oil, and potassium hydroxide). The initial WCR formulation doubled larval weight gain as compared to larvae reared on the SCR formulation (Pleau et al. 2002).

We previously developed an improved formulation for WCR larvae, hereafter referred to as "WCRMO-1" that was an optimization of the composition of ingredients in the initial WCR formulation (Huynh et al. 2017). Our improved diet was created by optimizing the key ingredients (corn root powder, wheat germ and casein) in the initial WCR formulation based on integrated evaluation of multiple larval developmental traits (weight, survival, and molting). The WCRMO-1 formulation supported approximately 99\% of larvae for both molting and survival and further doubled the weight of larvae compared to larvae reared on the initial WCR formulation after 11 days (Huynh et al. 2017).

Previous research in developing a diet for WCR larvae documented an important role of corn root powder as a key ingredient in the WCR diet that had significant impact on overall larval performance (survival, weight and molting) (Pleau et al. 2002, Huynh et al. 2017). Currently, corn root powder is the only ingredient that is not commercially available and specialized equipment (grinders [ZM200, Retsch, Haan, Germany] and lyophilizers [benchtop pro with omnitronics, SP Scientific, Stone Ridge, NY]) needed to make it are expensive, making it a limiting factor in the use of artificial diets for WCR larvae and practically unavailable to 
smaller companies, academics, and government researchers. If all of the ingredients in the rootworm diet could be purchased, more small companies and research groups could participate in rootworm research, which could expedite the development of new management tools. Since the corn root powder made for our original diet (Huynh et al. 2017) came from Monsanto, in this study, we verified that the milling process to produce corn root powder at the Biological Control of Insects Research Laboratory (BCIRL) (USDA-ARS, Columbia, MO) could be used with proprietary seed from Monsanto and that Viking seed could be substituted for Monsanto proprietary seed, which was milled by Monsanto and BCIRL.

The natural host material might contain a complex of chemicals and serve as needed micronutrient sources, token stimuli, or cryptic nutrients making natural host plants desirable in a diet (Cohen 2015). Corn roots not only contain feeding cues of WCR larvae, which are a combination of three sugars, glucose: fructose: sucrose plus one free fatty acid (oleic or linoleic acid) (Bernklau and Bjostad 2008), but also host recognition cues (Bernklau et al. 2015) and repellents (Bernklau et al. 2016). Once the factors from corn root powder can be extracted and identified while eliminating inessential components of corn root powder to the rootworm, then perhaps all ingredients for the WCR diet could be purchased. Toward a goal of developing an accessible diet for WCR larvae, we verified the role of corn root powder as micronutrients in diet formulations for WCR larvae and evaluated the possibility to extract the micronutrients based on integrated evaluation of three life history parameters (survival, weight and molting). 


\section{Material and methods}

\section{Insects}

WCR eggs (a non-diapausing strain) were obtained from the USDA-ARS laboratories in Columbia, $\mathrm{MO}$ and Brooking, SD. Egg plates were incubated in an incubator at $25^{\circ} \mathrm{C}$ in complete darkness. Once egg hatch started, the soil was removed by rinsing with water in a 60 mesh sieve (Hogentogler \& Co. Inc., Columbia, MD). The eggs free of soil were poured into a beaker and surface-treated using a procedure described by Pleau et al. (2002). Briefly, the eggs were exposed to undiluted lysol® (Reckitt Benckiser, LLC, Parsippany, NJ) for 3 min, and then triple rinsed with distilled water after removing the supernatant. Next, the remaining eggs were treated with 10\% formalin (HT501128, Sigma Aldrich, St. Louis, MO) for 3 min, and then triple rinsed with distilled water after removing the supernatant again. Finally, the remaining eggs were distributed to a coffee filter paper (Pure Brew, Rockline Industries, Sheboygan, WI) using a $1 \mathrm{ml}$ disposable pipette (13-711-9a, Fisher Scientific, Pittsburg, PA). The coffee paper was dried out for 2 min and then placed inside a 16 oz. cup with a lid (LG8RB-0090 \& DM16R-0090, Solo Cup Company, Lake Forest, IL). For ventilation, several holes made in the lid using number zero insect pin. The egg container was kept at $25^{\circ} \mathrm{C}$ in darkness. Neonates (within $24 \mathrm{~h}$ and hatching within 2 days) were used for insect bioassay.

\section{Corn root extraction}

Untreated, dry Viking seeds (Viking 60-01N, Albert Lea Seed, Albert Lea, MN) were soaked for $24 \mathrm{~h}$ in tap water, rinsed, and germinated on moist blotter paper (Steel Blue, Anchor Paper Company, St. Paul, MN). The seeds were kept at $25^{\circ} \mathrm{C}$ in closed polyethylene containers $(20 \times 40 \times 5 \mathrm{~cm})$. Roots $(6 \mathrm{~g})$ were harvested from 5 -d-old germinating seeds, placed in a $150 \mathrm{ml}$ 
Erlenmeyer flask, and $100 \mathrm{ml}$ of methanol was added. After $1 \mathrm{~h}$, the liquid was filtered (Whatman no. 1, 1004-090, Springfield Mill, Maidstone, Kent, England) into a $500 \mathrm{ml}$ round bottom flask and the extract was concentrated to approximately $1 \mathrm{ml}$ using a rotary evaporator. The concentrated liquid was transferred to a clean glass vial (4 ml, 66011-041, VWR International, West Chester, PA), the flask was rinsed twice with $1.5 \mathrm{ml}$ methanol to collect remaining residue from the walls of the flask and the rinses were added to the concentrated solution. The extract was evaporated under a stream of nitrogen to remove the methanol (approximately $0.5 \mathrm{ml}$ final volume). The extracted roots were air-dried overnight, weighed, and the extract concentration was adjusted (with methanol) to 0.1 gram equivalents dry root per ml. Agar (5 g, A7002, Sigma-Aldrich) was placed in a glass container and the extract (2.5 ml total) was added $0.5 \mathrm{ml}$ at a time, shaking well after each addition. The agar treated with extracted corn roots was spread out on a Teflon sheet and allowed to dry $48 \mathrm{~h}$ before being stored in an air-tight container.

\section{Corn root powder}

Corn root powder from Monsanto proprietary seed and Viking seed was provided by both Monsanto (Monsanto, St. Louis, MO) and produced by the Biological Control of Insects Research Laboratory (USDA-ARS, Columbia, MO). Corn root powder provided by Monsanto from Viking seed and Monsanto seed was used 7 months and 12 months after receipt, respectively, whereas corn root powder made by BCIRL from Viking seed and Monsanto seed was used 3 months and 1 month, respectively, after being produced. All were stored at $-20^{\circ} \mathrm{C}$ until use. Briefly, at BCIRL, corn seeds (500 g) were washed in $1000 \mathrm{ml}$ beaker via exposure to $10 \%$ bleach $\left(\right.$ Clorox $^{\circledR}$ Regular Bleach, Clorox, Oakland, CA) solution plus $0.01 \%$ Tween $^{\mathrm{TM}} 20$ 
(BP337, Fisher Scientific) for $15 \mathrm{~min}$, triple rinsed with distilled water, and then incubated at $24^{\circ} \mathrm{C}$ for $24 \mathrm{~h}$. The seeds were placed in a straight line on top of germination paper (SD3815L, Anchor Paper Co., Saint Paul, Minnesota) with 50 seeds per paper and the papers were fold over the seeds and rolled tightly, secured with a rubber band to make a 'rag doll'. The rag dolls were placed upright in a 5-gallon bucket (40 rolls per bucket) containing distilled water to encourage downward growth of root tissue. The bucket was kept in an incubator at $27^{\circ} \mathrm{C}$ with $\mathrm{RH}$ of $70 \%$ and watered every two days. After 7 days, corn roots were collected by cutting paper rolls below the rubber band with a guillotine type paper cutter. The corn roots were then cut into less than 2 cm using a cutter (American Metalcraft, Inc., Franklin Park, IL), placed in a tray covered by liquid nitrogen, and then dried in a lyophilizer (benchtop pro with omnitronics, SP Scientific, Stone Ridge, NY) at $35^{\circ} \mathrm{C}$ for 7 days. After 7 days of lyophilizing, the dry roots were milled into powder using an ultra-centrifugal mill (ZM200, Retsch, Haan, Germany) at $14500 \mathrm{rpm}$.

\section{Diet preparation}

The diet was poured as descriptions by Huynh et al. (2017) modified from Pleau et al. (2002). All dry ingredients i.e. casein (1100, Bio-Serv, Flemington, NJ), cholesterol (C8503, Sigma-Aldrich), cellulose (3425, Bio-Serv), corn root powder (BCIRL and Monsanto), methyl paraben (H5501, Sigma-Aldrich), salt mix (F8680, Bio-Serv), sorbic acid (S1626, SigmaAldrich), sucrose (04821721, MP Biomedicals, Santa Ana, CA), vitamin mix (V1007, SigmaAldrich), and wheat germ (1661, Bio-Serv) were weighed and put in a plastic bag, except for agar (A7002, Sigma-Aldrich). Agar solution (agar plus distilled water) in a $600 \mathrm{ml}$ beaker was boiled in a microwave for $2 \mathrm{~min}$, and then poured into a blender (Hamilton Beach, Inc., Model 51101BZ) in a biosafety cabinet (Nuaire, Plymouth, MN). The dry ingredients were added into 
the blender and mixed thoroughly for 30 seconds. Next, liquid components i.e. linseed oil (430021, Sigma-Aldrich), wheat germ oil (W1000, Sigma-Aldrich), streptomycin (612240500, Across, Morris Plains, NJ), chlortetracycline (C4881, Sigma-Aldrich), and green food coloring (Bulter, Lancaster, PA) were added into the blender and mixed thoroughly for 30 seconds. The $\mathrm{pH}$ of diet was increased to $\mathrm{pH}$ of 9 by an addition of $\mathrm{KOH} 10 \%(w / v)(\mathrm{P} 250$, Fisher Scientific) and monitored using indicator strips (Whatman). The diet mixture was then poured into a low sided $700 \mathrm{ml}$ beaker placed on a hot plate (Cimarec ${ }^{\mathrm{TM}}$, Thermo Scientific) at $65^{\circ} \mathrm{C}$ and mixed using a stir bar. The mixture was dispensed into a 96-well plate (3370, Corning Inc., Corning, NY) using a repeated pipetted (200 $\mu 1$ per well) (Eppendorf repeater plus). The diet plate was allowed to evaporate excess moisture in the biological cabinet for $10 \mathrm{~min}$, stored in a refrigerator at $4{ }^{\circ} \mathrm{C}$ within a week. For diet bioassays, each formulation tested was randomly assigned in a $12-$ well row of the 96-well plate and replicated at least 4 times in different diet plates.

\section{Larval artificial diet bioassays}

The bioassays were conducted as described in Huynh et al. (2017). All materials used in larval handling were sterilized via exposure UV light for $10 \mathrm{~min}$ in biological cabinet. One neonate larva ( $<24 \mathrm{~h}$ after hatching) was infested to each well using a paintbrush. A sealing film (TSS-RTQ-100, Excel Scientific, Inc., Victorville, CA) was used to cover the plate and a hole per well to exchange air ventilation was made using a number zero insect pin. The plates were kept at $25^{\circ} \mathrm{C}$ in darkness for 15 days. Larval weight, survival and evidence of diet contamination were recorded at 15 days. For dry weight, surviving larvae were collected and pooled per replicate into $95 \%$ ethanol, and then dried at $50^{\circ} \mathrm{C}$ for 2 days in an oven $(602752$, Blue M Therm Dry Bacteriological Incubator). Dry larvae per replicate were weighed using a micro balance 
(MSU6.6S-000-DM, Sartorius Lab Instruments GmbH \& Co. KG, Goettingen, Germany). To determine differences in larval molting to $2^{\text {nd }}$ instar and $3^{\text {rd }}$ instar, larval molting to $2^{\text {nd }}$ instar was recorded at 10 days and larval molting to $3^{\text {rd }}$ instar and larval incomplete molting to $2^{\text {nd }}$ instar defined as an inability to complete removal of larval skin were recorded at 15 days.

\section{Diet bioassays with corn root extract}

To determine if essential nutrients in corn roots could be extracted, diets differing in types of corn root extraction including corn root powder, dry corn root, extracted corn roots and agar treated with extracted corn roots, were tested for their effect on larval performance (survival, weight, and molting). The proportions of these ingredients were at $1.5 \%(\mathrm{w} / \mathrm{w})$, the same proportion of corn root powder and agar in the WCRMO-1 diet (Huynh et al. 2017). In formulations with agar treated with extracted corn roots and without corn root powder, the proportion of water was increased to $1.5 \%(\mathrm{w} / \mathrm{w})$. Other ingredients were the same as in the WCRMO-1 formulation.

\section{Diet bioassays with corn root powder as a major nutritional source}

Corn root powder was demonstrated as the key ingredient that was used in combination with major nutritional components (e.g., wheat germ, casein, sucrose, linseed oils) (Pleau et al. 2002, Huynh et al. 2017). To determine if optimizing corn root powder alone while eliminating other macronutrient sources could be used for WCR larval growth, we varied corn root powder at several concentrations (i.e., $0 \%, 1 \%, 1.5 \%, 3 \%, 6 \%$, and $10 \% \mathrm{w} / \mathrm{w}$ ) and added vitamin mix, diet preservatives, food coloring, agar, and water and excluding other nutritional components (e.g., wheat germ, casein, sucrose, lipid components). The maximum concentration 
of corn root powder in these agar-based diets was $10 \% \mathrm{w} / \mathrm{w}$. The amount of these additional ingredients were the same in the WCRMO-1 diet (Huynh et al. 2017), except for the proportions of water that were changed to accommodate changes in the proportions of corn root powder (Table 8).

\section{Diet bioassays with corn root powder from different seeds and suppliers}

To verify the milling process producing corn root powder between Monsanto and BCIRL and the substitution of Viking seed for Monsanto proprietary seed, four diets differing in corn root powder made by Monsanto and BCIRL from both Monsanto proprietary seed and Viking seed were compared for their support of larval performance (survival, weight and molting). The proportions of corn root powder and remaining ingredients were as the same in WCRMO-1 diet (Huynh et al. 2017).

\section{Statistical analyses}

Larval survivorship and molting were generated by dividing the number of surviving larvae and molted larvae by the number of initial larvae within a replicate and multiplying by 100. Weight data was generated by dividing total dry weight by the number of surviving larvae within a replicate. Larval performance (larval survival, weight, molt to $2^{\text {nd }}$ instar, incomplete molt to $2^{\text {nd }}$ instar, and molt to $3^{\text {rd }}$ instar) were analyzed as a randomized complete block design using PROC MIXED in SAS. 


\section{Results}

\section{Diet bioassays with corn root extraction}

Clearly, factors required for molting were extractable. There was no significant difference between the dry root treatment and the extract from dry roots $\left(p<0.0001, F_{4,28}=\right.$ 22.31, Fig. 6C). Larval dry weight $\left(p<0.0001, F_{4,28}=21.76\right.$, Fig. 6B was better on the dry corn root treatment than the extracts indicating that the solvent did not extract all relevant nutrients (Fig. 6B). Corn root powder was better than the dried corn roots for most factors evaluated including survivorship $\left(p<0.0001, F_{4,28}=21.76\right.$, Fig. $\left.6 \mathrm{~A}\right)$. Molting to the $3^{\text {rd }}$ instar, in particular, was best with corn root powder $\left(p<0.0001, F_{4,28}=20.39\right.$, Fig. $\left.6 \mathrm{E}\right)$. Exclusion of dry corn roots or corn root powder also led to a significant increase in the percentage of larvae with incomplete molting to the $2^{\text {nd }}$ instar to $15 \mathrm{~d}$ compared to the diet with agar treated with extracted corn roots $\left(p<0.0001, F_{4,28}=13.91\right)$ whereas no incomplete molting was seen in other treatments (Fig. 6D).

\section{Diet bioassays with corn root powder as the major nutritional source}

Larval survivorship to $15 \mathrm{~d}$ was significant difference in diets containing corn root powder as the main nutrient source $\left(p<0.0001, F_{5,15}=33.41\right)$. An increase in proportions of corn root powder resulted in an increase in survival. No larva survived in diets with corn root powder at $0 \%$ and $1 \%$ while only $4.1 \%$ larvae survived on a diet with $1.5 \%$ corn root powder to $15 \mathrm{~d}$ (Fig. 7A). There was no significant difference among these diets whereas a diet with corn root powder at $3 \%$ produced more larvae compared with the diet with corn root powder at $1.5 \%$. More surviving larvae in diets with corn root powder at $6 \%$ and $10 \%$ compared to other diets, and there was no significant difference among these diets. Larval dry weight to $15 \mathrm{~d}$ ranged from $0.001 \mathrm{mg} / \mathrm{larva}$ for the diet with $1.5 \%$ corn root powder to $0.012 \mathrm{mg} / \mathrm{larva}$ for the diet with 
6\% corn root powder (Fig. 7B), which was at least 43 fold lower compared to larvae feeding on a diet with corn root powder combined with macronutrient ingredients (e.g. wheat germ, casein, sucrose) (Fig. 6B). Diets with corn root powder at 6\% and 10\% had significant higher larval weight than other diets $\left(p<0.0001, F_{5,15}=38.48\right)$. Larval weight for the diet with corn root powder at $3 \%$ was significantly higher than the diets with corn root powder at $1.5 \%$. No larva that molted to the $2^{\text {nd }}$ instar was seen within $15 \mathrm{~d}$ in all diets.

\section{Diet bioassays with corn root powder form different seeds and suppliers}

Larval survivorship to $15 \mathrm{~d}$ was good on all diets differing in corn root powder from different seeds or suppliers, ranged from $96.5 \%$ for a diet with Viking seed milled by Monsanto to $100 \%$ for a diet with Viking seed milled by BCIRL (Fig. 8A) and there was no significant difference among all diets $\left(p=0.1884, F_{3,21}=1.75\right)$. For larval dry weight to $15 \mathrm{~d}$, there was no significant difference among the diets with Viking seed milled by BICRL and Monsanto and the diet with Monsanto seed milled by BICRL while the diet with Monsanto seed milled by Monsanto had the smallest larval weight $(0.52 \mathrm{mg} / \mathrm{larva})$ as compared to other diets $(p=0.0028$, $F_{3,21}=6.49$ ), ranged from $0.67 \mathrm{mg} /$ larva to $0.78 \mathrm{mg} /$ larva (Fig. $8 \mathrm{~B}$ ). Percentages of larval molted to the $2^{\text {nd }}$ instar to $10 \mathrm{~d}$ on all diets were high, ranging from $95.1 \%$ to $97.5 \%$ and there was no significant difference between all diets $\left(p=0.7994, F_{3,21}=0.34\right)$ (Fig. 8C). The percentages of larvae molted to the $3^{\text {rd }}$ instar within $15 \mathrm{~d}$ were significant differences among diets $(p=0.0191$, $F_{3,21}=4.12$ ). The diet with Monsanto seed milled by BCIRL produced significantly more $3^{\text {rd }}$ instar larvae compared to the diets with Monsanto and Viking seeds milled by Monsanto whereas the percentage of the $3^{\text {rd }}$ instar larvae reared on the diet with Viking seed milled by BCIRL was significantly higher than that for the diet with Monsanto seed milled by Monsanto (Fig. 8D). 
Overall, the diets differing in corn root powder sources exhibited no significant difference in larval survivorship to $15 \mathrm{~d}$ and molting to the $2^{\text {nd }}$ instar while the differences were seen in larval weight and molting to the $3^{\text {rd }}$ instar after $15 \mathrm{~d}$ of feeding. Corn root powder made by BCIRL from Monsanto seed and Viking seed was used at 1 month and 3 months after being produced, respectively while it provided by Monsanto from Monsanto seed and Viking seed was used at 12 months and 7 months after receipt, respectively.

\section{Discussion}

Here we demonstrated that the presence of factors from corn roots significantly increase survivorship, larval weight, and molting are found in corn roots (Fig. 6). Adding corn root extracts to a base diet for WCR significantly improved all of measured developmental traits of larval performance compared to larvae reared on the base diet without corn root extracts. Importantly, there was no significant difference between the extract and the same amount of the corn root tissue that they came from when evaluating molting to the second instar (Fig. 6C). Larval survivorship and larval weight were better than the extracts indicating that the solvent did not extract all relevant nutrients (Figs. 6A-B). Corn root powder was even better than the dried corn roots for most factors evaluated, but may or may not have been directly comparable. Our results suggest it is possible to isolate and identify the essential factors in corn roots that assist in growth and development. Several insects have been reared successfully on artificial diets by adding natural host material into a base diet consisting of major nutritional and textural features (e.g., soy flour, wheat germ, or casein) (Lindig and Malone 1973, Ito et al. 1975, Brun et al. 1993, Blossey et al. 2000). Corn roots contain host recognition cues and feeding stimulants, but also have a repellent compound for WCR larvae (Bernklau and Bjostad 2008, Bernklau et al. 
2015, Bernklau et al. 2016). Since corn root powder is the only ingredient in the WCR formulation that is not commercially available, the identification of the essential factors in corn roots will assist formulation of an open access diet for which all of ingredients can be purchased.

Corn root powder was optimized in combination with key diet components (wheat germ and casein) in the WCR formulation (Huynh et al. 2017). Our results revealed that corn root powder alone without the addition of several macronutrient sources (e.g., wheat germ, casein, sucrose, linseed oil, wheat germ oil) failed to develop a diet that supports larval growth successfully compared to a diet with these factors. The maximum concentration of corn root powder added in agar-based corn root powder diets while eliminating several nutritional components (i.e., wheat germ, casein, sucrose, linseed oil, wheat germ oil, cellulose, and salt mix) from the WCRMO-1 (Huynh et al. 2017) was seen at 10\% w/w. Larvae survived without molting to $15 \mathrm{~d}$ of feeding on the agar-based corn root powder diets containing at least $1.5 \%$ corn root powder (Fig. 7). The poor performance of larvae fed the agar-based corn root powder diets is possibly due to inadequate nutrition in the diet. Research with lepidopteran species showed that inadequate dietary protein causes inhibition of larval molting of larvae due to insufficient production of ecdysteroid in the hemolymph of larvae (Hamano et al. 1994).

Diet assays for WCR larvae typically run up to $10 \mathrm{~d}$ (Pleau et al. 2002, Siegfried et al. 2005, Ludwick et al. 2018). All diets with differing corn root powder sources supported over 95\% of larvae for survival and molting to the $2^{\text {nd }}$ instar to $10 \mathrm{~d}$ of feeding (Fig. 8 ) while data for weight to $10 \mathrm{~d}$ was not available because weight data was only recorded at the end of experiments $(15 \mathrm{~d})$. These results suggest the milling process to making corn root powder was not significantly different between Monsanto and BCIRL, and Viking seed can be used as a substitution for the industry proprietary seed. Interestingly, the differences in the $3^{\text {rd }}$ instar for 
weight and molting were seen in different diets. This is possibly due to shelf life of corn root powder that might affect the nutritional quality of corn root powder. There was a correlation between time of storage and the nutritional condition of corn root powder. Corn root powder from Monsanto seed and Viking provided by Monsanto was used at 12 months and 7 months of receipt, respectively. The diets with these corn root powder exhibited lower support of larval performance compared to the diets with corn root powder used within 3 months of storage (Fig 8).

Larvae were capable of developing to the $3^{\text {rd }}$ instar in 15-day bioassays, which allowed the detection of differences in multiple larval development stages. Data collection from the 5-day and 6-day diet bioassays for WCR initially included larval weight and survival while ignoring the information about molting (Pleau et al. 2002, Siegfried et al. 2005). The limited bioassay duration was possibly due to contamination, which was reported as a major issue of diet bioassays (Pereira et al. 2016). By minimizing contamination through clean laboratory practices as described by Huynh et al. (2017) and Ludwick et al. (2018), the length of diet bioassays for WCR testing was extended to $10 \mathrm{~d}$ (Ludwick et al. 2018) or $11 \mathrm{~d}$ (Huynh et al. 2017), which allowed the determination of differences in larval performance (weight, molt, and survival) to the $2^{\text {nd }}$ instar. In this manuscript, we had $<1 \%$ contamination during all experiments. Similarly low contamination rates were reported in Huynh et al. (2017) and Ludwick et al. (2018). The ability to conduct a longer bioassay would facilitate for experiments that requires longer periods (e.g. dsRNA) and for continuous rearing for WCR that allows optimizing labor and cost for rearing while avoiding possible physical damage and contamination due to transferring. 
Table 8. Components in diets used to rear western corn rootworm larvae in corn root powder experiment.

\begin{tabular}{ll}
\hline \multicolumn{1}{c}{ Components } & Amount \\
\hline Variable components & \\
1. Corn root powder & $0-9 \mathrm{~g}$ \\
2. Distilled water & $75-84 \mathrm{ml}$ \\
Constant components & \\
3. Agar & $1.5 \mathrm{~g}$ \\
4. Chlortetracycline $(10 \mathrm{mg} / \mathrm{ml})$ & $6.4 \mathrm{mg}$ \\
5. Food coloring & $6.4 \mathrm{mg}$ \\
6. Methyl paraben & $0.1 \mathrm{~g}$ \\
7. Potassium hydroxide $(10 \%)$ & $3.5 \mathrm{ml}$ \\
8. Sorbic acid & $6.4 \mathrm{mg}$ \\
9. Streptomycin $(12.8 \mathrm{mg} / \mathrm{ml})$ & $6.4 \mathrm{mg}$ \\
10. Vanderzant vitamin $\mathrm{mix}$ & $0.90 \mathrm{~g}$ \\
\hline
\end{tabular}


(A)

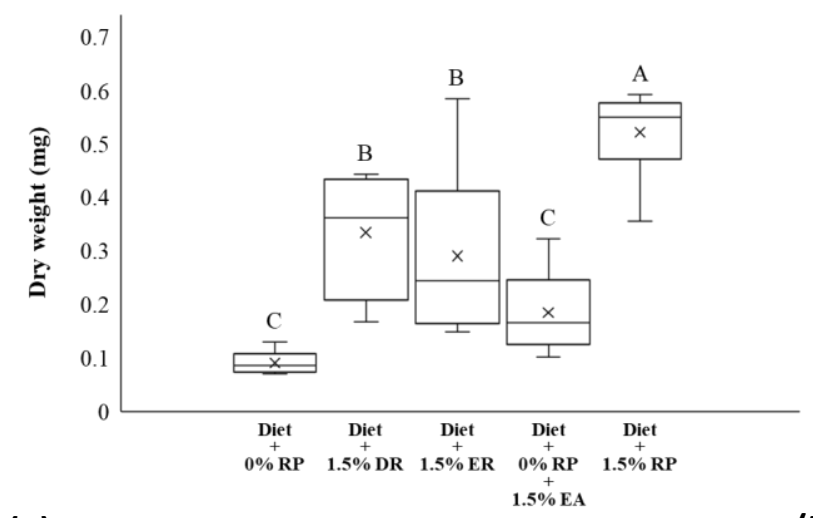

(C)
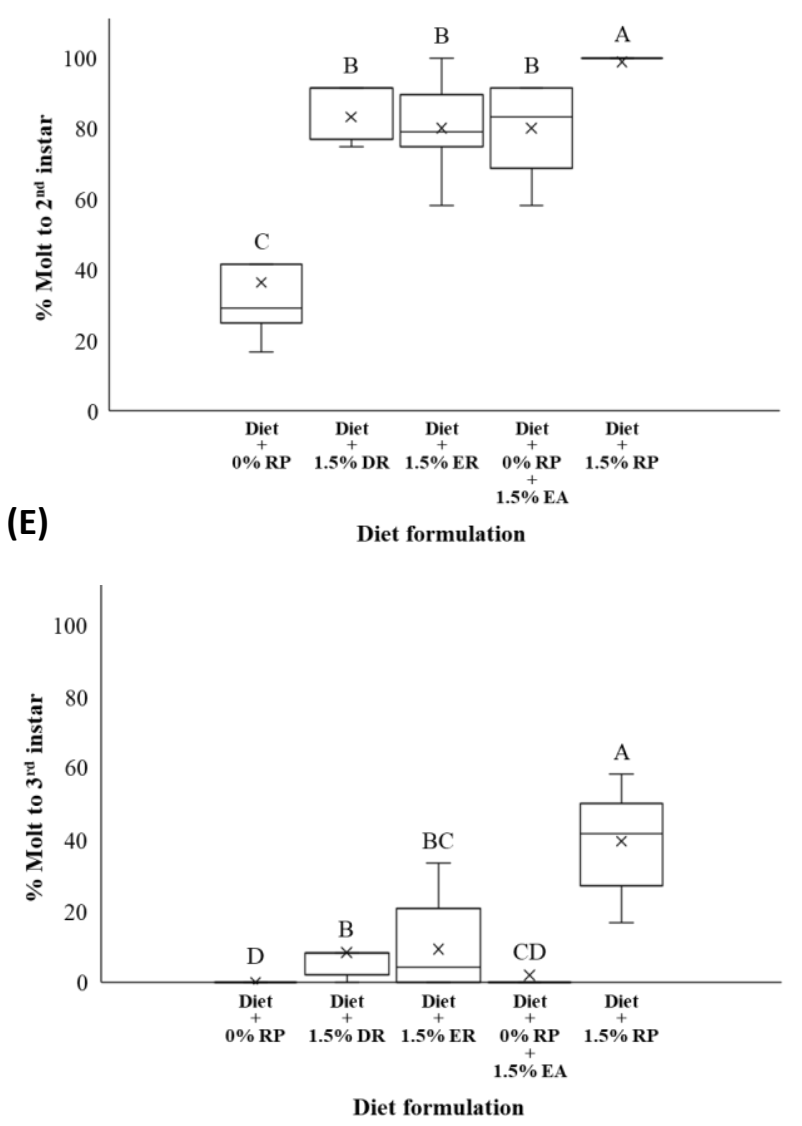

(B)
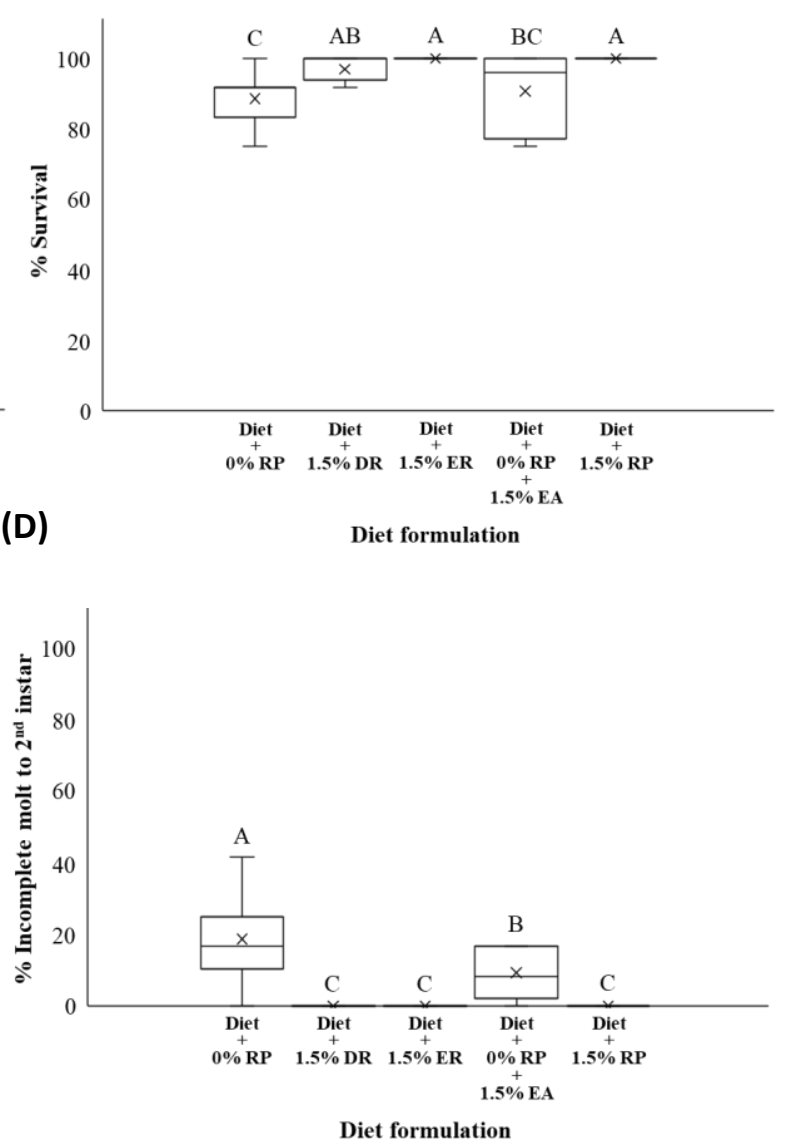

Figure 6. Survival (a), average dry weight (b), molt to $2^{\text {nd }}$ instar (c), incomplete molt to $2^{\text {nd }}$ instar (d), molt to $3^{\text {rd }}$ instar (e) of western corn rootworm larvae reared on diets containing different types of corn roots for 15 days. RP: corn root powder, DR: dry corn root, ER: extracted corn root, EA: agar treated with extracted corn root. Other ingredients were same as in WCRMO-1 formulation (Huynh et al. 2017). Boxes followed by different letters are significantly different $(p<0.05)$. 
(A)

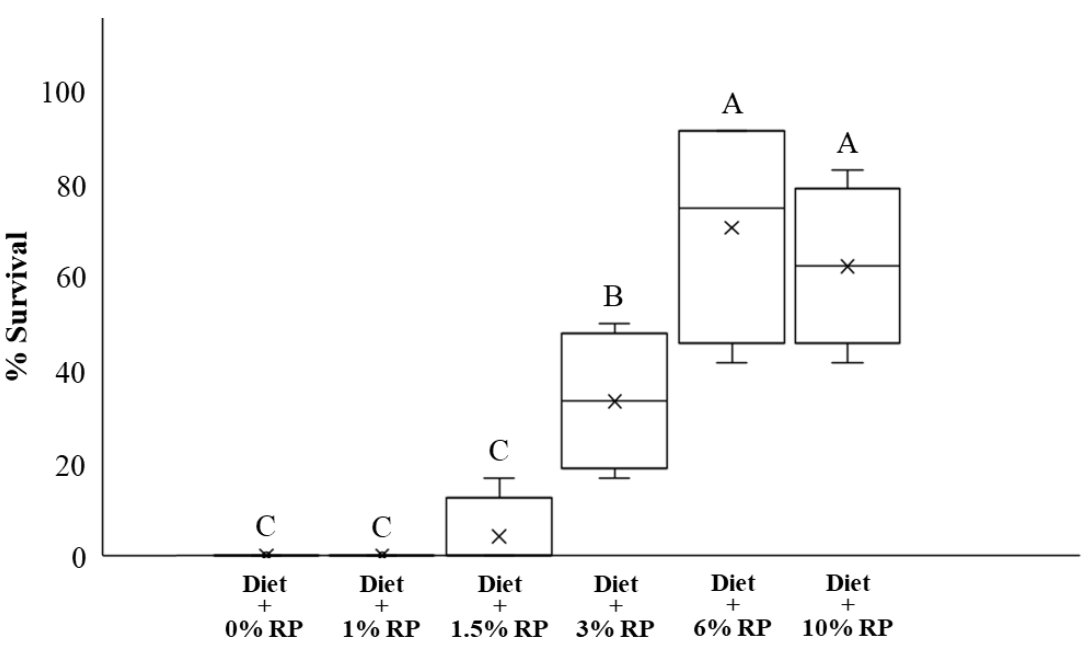

(B)

Diet formulation

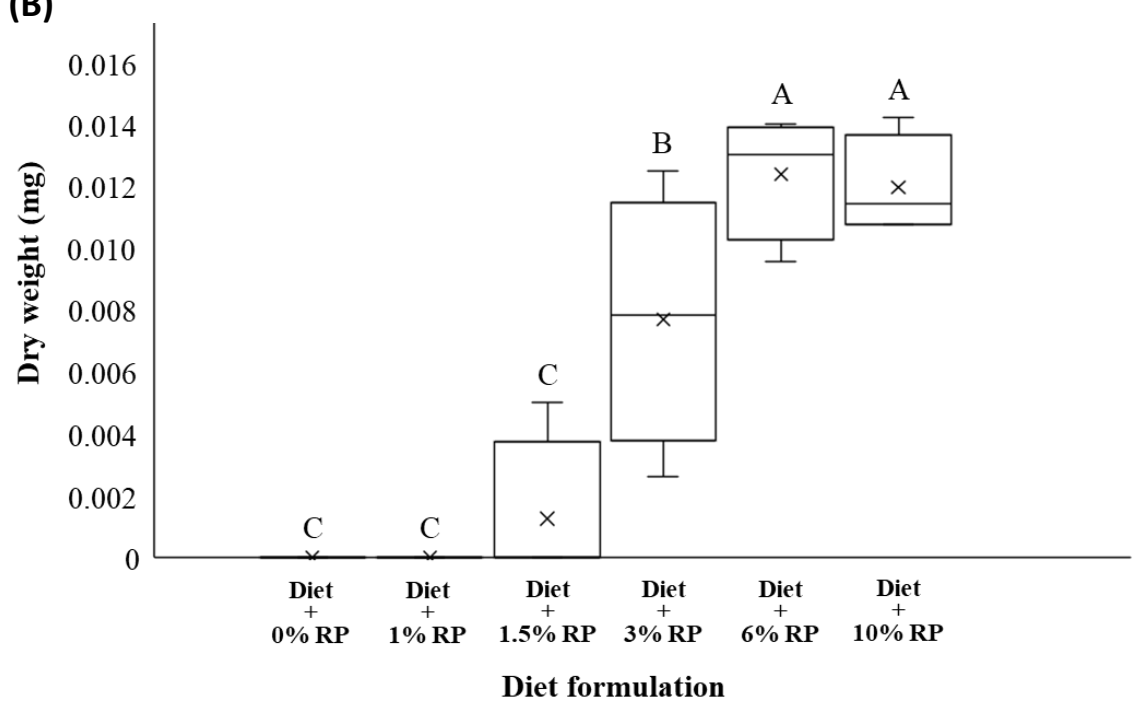

Figure 7. Survival (a), and average dry weight (b) of western corn rootworm larvae reared on diet formulations containing corn root powder as the major macronutrient component for 15 days. Other ingredients included vitamin mix, cholesterol and diet preservatives. Boxes followed by different letters are significantly different $(p<0.05)$. 
(A)

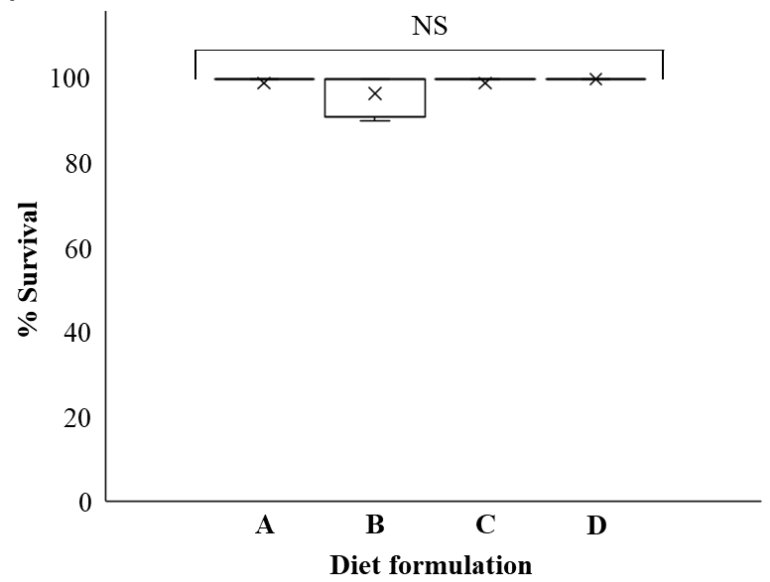

(C)

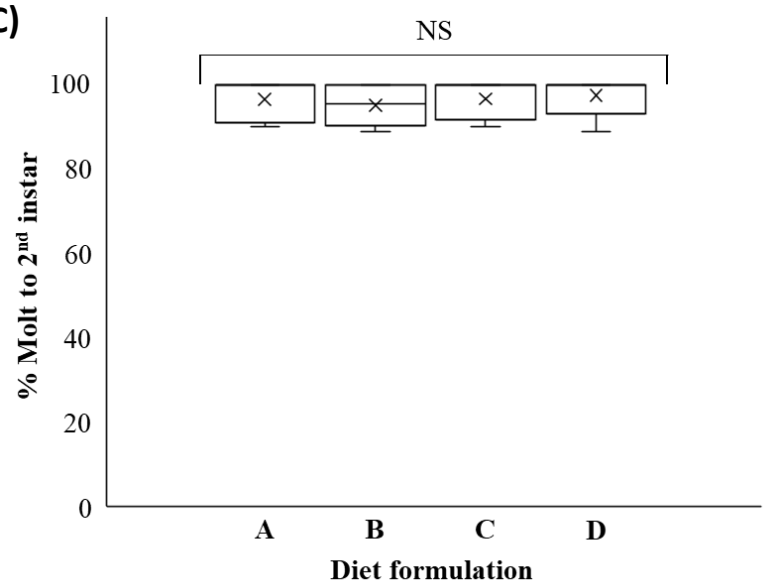

(B)

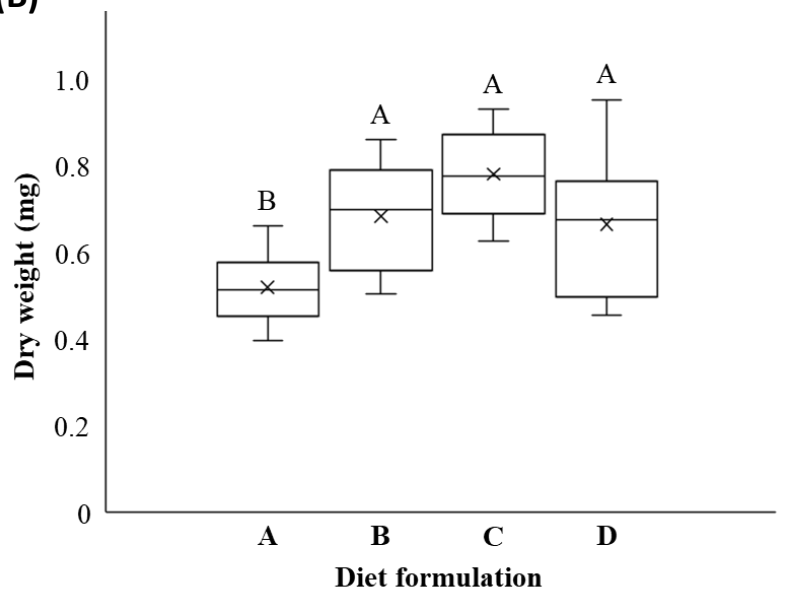

(D)

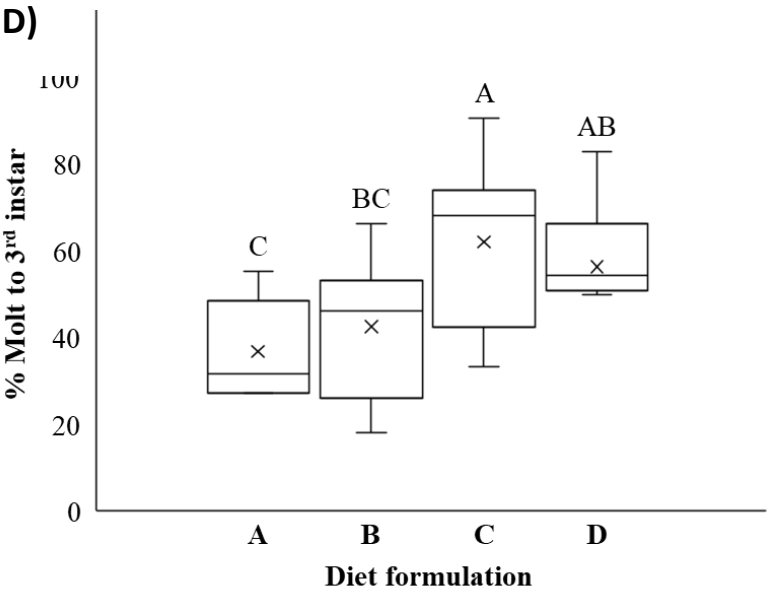

Figure 8. Survival (a), average dry weight (b), molt to $2^{\text {nd }}$ instar (c), molt to $3^{\text {rd }}$ instar (d) of western corn rootworm larvae reared on diet formulations differing in corn root powder sources for $15 \mathrm{~d}$. Diet A and diet B contained corn root powder provided by Monsanto from Monsanto seed and Viking seed, respectively while corn root powder made by BCIRL from Monsanto seed and Viking seed was included in $\operatorname{diet} \mathrm{C}$ and $\operatorname{diet} \mathrm{D}$, respectively. Other ingredients in all diets were same as in WCRMO-1 formulation (Huynh et al. 2017). Boxes followed by different letters are significantly different $(p<0.05)$. 


\section{References}

Ball, H. J., and G. T. Weekman. 1963. Differential resistance of corn rootworms to insecticides in Nehraska and adjoining States. J. Econ. Entomol. 56: 553-555.

Bernklau, E. J., and L. B. Bjostad. 2008. Identification of feeding stimulants in corn roots for western corn rootworm (Coleoptera: Chrysomelidae) larvae. J. Econ. Entomol. 101: 341351.

Bernklau, E. J., B. E. Hibbard, A. P. Norton, and L. B. Bjostad. 2016. Methyl anthranilate as a repellent for western corn rootworm larvae (Coleoptera: Chrysomelidae). J. Econ. Entomol. 109: 1683-1690.

Bernklau, E. J., B. E. Hibbard, D. L. Dick, C. D. Rithner, and L. B. Bjostad. 2015. Monogalactosyldiacylglycerols as host recognition cues for western corn rootworm larvae (Coleoptera: Chrysomelidae). J. Econ. Entomol. 108: 539-548.

Blossey, B., D. Eberts, E. Morrison, and T. R. Hunt. 2000. Mass rearing the weevil Hylobius transversovittatus (Coleoptera: Curculionidae), biological control agent of Lythrum salicaria, on semiartificial diet. J. Econ. Entomol. 93: 1644-1656.

Branson, T. F., and J. L. Krysan. 1981. Feeding and oviposition behavior and life cycle strategies of Diabrotica: an evolutionary view with implications for pest management. Environ. Entomol. 10: 826-831.

Brun, L. O., V. Gaudichon, and P. Wigley. 1993. An artificial diet for continuous rearing of the coffee berry borer, Hypothenemus hampei (Ferrari) (Coleoptera: Scolytidae). Int. J. Trop. Insect Sci. 14: 585-587.

Cohen, A. C. 2015. Insect diets: science and technology, 2nd ed., Taylor \& Francis Group.

Culy, M. D., C. R. Edwards, and J. R. Cornelius. 1992. Effect of silk feeding by western corn rootworm (Coleoptera: Chrysomelidae) on yield and quality of inbred corn in seed corn production fields. J. Econ. Entomol. 85: 2440-2446.

EPA. 2016. EPA docket for corn rootworm resistance management and framework for Bt corn. In U. S. E. P. Agency [ed.]. United States Environmental Protection Agency, Washington, DC, USA.

Gassmann, A. J., J. L. Petzold-Maxwell, R. S. Keweshan, and M. W. Dunbar. 2011. Fieldevolved resistance to Bt maize by western corn rootworm. PloS one 6: e22629. 
Gray, M. E., T. W. Sappington, N. J. Miller, J. Moeser, and M. O. Bohn. 2009. Adaptation and invasiveness of western corn rootworm: intensifying research on a worsening pest. Ann. Rev. Entomol. 54: 303-321.

Hamano, K., A. Ikeda, and W. Shen. 1994. Relationship between food consumption and molting of the silkworm, Bombyx mori. Proc. Jpn. Acad., Series B 70: 146-150.

Huynh, M. P., L. N. Meihls, B. E. Hibbard, S. L. Lapointe, R. P. Niedz, D. C. Ludwick, and T. A. Coudron. 2017. Diet improvement for western corn rootworm (Coleoptera: Chrysomelidae) larvae. PloS one 12: e0187997.

Ito, T., Y. Horie, and S. Nakasone. 1975. Deterrent effect of soybean meal on feeding of the silkworm, Bombyx mori. J. Ins. Physiol 21: 995-1006.

Levine, E., J. L. Spencer, S. A. Isard, D. W. Onstad, and M. E. Gray. 2002. Adaptation of the western corn rootworm to crop rotation: evolution of a new strain in response to a management practice. Am. Entomol. 48: 94-117.

Lindig, O., and O. Malone. 1973. Oviposition of boll weevils fed diets containing germinated cottonseed puree or cottonseed meats puree. J. Econ. Entomol. 66: 566-567.

Ludwick, D. C., L. N. Meihls, K. R. Ostlie, B. D. Potter, L. French, and B. E. Hibbard. 2017. Minnesota field population of western corn rootworm (Coleoptera: Chrysomelidae) shows incomplete resistance to Cry34Ab1/Cry35Ab1 and Cry3Bb1. J. Appl. Entomol. 141: 28-40.

Ludwick, D. C., L. N. Meihls, M. P. Huynh, A. E. Pereira, B. W. French, T. A. Coudron, and B. E. Hibbard. 2018. A new artificial diet for western corn rootworm larvae is compatible with and detects resistance to all current Bt toxins. Sci. Rep. 8: 5379.

Marrone, P. G., F. D. Ferri, T. R. Mosley, and L. J. Meinke. 1985. Improvements in laboratory rearing of the southern corn rootworm, Diabrotica undecimpuncta howardi Barber (Coleoptera: Chrysomelidae), on an artificial diet and corn. J. Econ. Entomol. 78: 290293.

Meinke, L. J., B. D. Siegfried, R. J. Wright, and L. D. Chandler. 1998. Adult susceptibility of Nebraska western corn rootworm (Coleoptera: Chrysomelidae) populations to selected insecticides. J. Econ. Entomol. 91: 594-600.

Metcalf, R. L. 1986. Methods for the study of pest Diabrotica (ed. by J. L. Krysan and T.A. Miller), pp. vii-xv, Springer-Verlag, New York. 
Moeser, J., and S. Vidal. 2005. Nutritional resources used by the invasive maize pest Diabrotica virgifera virgifera in its new south-east-European distribution range. Ent. Exp. Appl. 114: 55-63.

Oyediran, I. O., B. E. Hibbard, T. L. Clark, and B. W. French. 2004. Selected grassy weeds as alternate hosts of northern corn rootworm (Coleoptera: Chrysomelidae). Environ. Entomol. 33: 1497-1504.

Oyediran, I. O., B. W. French, T. L. Clark, K. E. Dashiell, and B. E. Hibbard. 2008. Prairie grasses as hosts of the northern corn rootworm (Coleoptera: Chrysomelidae). Environ. Entomol. 37: 247-254.

Pereira, A. E., N. P. Carneiro, and B. D. Siegfried. 2016. Comparative susceptibility of southern and western corn rootworm adults and larvae to vATPase-A and Snf7 dsRNAs. J. RNAi and Gene Silencing 12: 528-535.

Pereira, A. E., D. Souza, S. N. Zukoff, L. J. Meinke, and B. D. Siegfried. 2017. Cross-resistance and synergism bioassays suggest multiple mechanisms of pyrethroid resistance in western corn rootworm populations. PloS one: 12(16): e0179311.

Pereira, A. E., H. Wang, S. N. Zukoff, L. J. Meinke, B. W. French, and B. D. Siegfried. 2015. Evidence of field-evolved resistance to bifenthrin in western corn rootworm (Diabrotica virgifera virgifera LeConte) populations in western Nebraska and Kansas. PloS one 10: e0142299.

Pleau, M. J., J. E. Huesing, G. P. Head, and D. J. Feir. 2002. Development of an artificial diet for the western corn rootworm. Entomol. Exp. Appl. 105: 1-11.

Rose, R. I., and J. M. McCabe. 1973. Laboratory rearing techniques for the southern corn rootworm. J. Econ. Entomol. 66: 398-400.

Siegfried, B. D., T. T. Vaughn, and T. Spencer. 2005. Baseline susceptibility of western corn rootworm (Coleoptera: Crysomelidae) to Cry3Bb1 Bacillus thuringiensis toxin. J. Econ. Entomol. 98: 1320-1324.

Sutter, G. R., J. L. Krysan, and P. L. Guss. 1971. Rearing the southern corn rootworm on artificial diet. J. Econ. Entomol. 64: 65-67. 
Zukoff, S. N., A. L. Zukoff, R. W. Geisert, and B. E. Hibbard. 2016. Western corn rootworm (Coleoptera: Chrysomelidae) larval movement in eCry3.1Ab+mCry3A seed blend scenarios. J. Econ. Entomol. 109: 1834-1845. 


\title{
CHAPTER IV: MIXTURE DESIGN AND RESPONSE SURFACE MODELING TO DEVELOP AN IMPROVED AND ACCESSIBLE DIET FOR WESTERN CORN ROOTWORM LARVAE
}

\begin{abstract}
The western corn rootworm (WCR), Diabrotica virgifera virgifera LeConte, is an important pest of maize (Zea mays L.) in the U.S Corn Belt and some parts of Europe. An artificial diet that supports WCR development similar to larval feeding on maize roots would facilitate resistance monitoring efforts and new research discoveries. Currently, artificial diets are used in diet assays to detect the susceptibility of WCR populations to Bacillus thuringiensis Berliner (Bt) and other toxins. Published WCR diets contain corn root powder, which is not available for purchase, limiting the practical use of the diets. We applied mixture designs combined with response surface modeling to develop a new diet formulation for WCR without corn root powder. We developed the new formulation by systematically exploring eight different protein ingredients from animal, plant, and yeast sources based on simultaneous evaluation of three life history parameters (weight, molting, and survival). This formulation (WCRMO-2) without corn root powder supported approximately $97 \%$ of larvae for both survival and molting, and increased larval weight gain after 10 days of feedings by 4-fold compared with the current best published diet. Other characteristics of WCRMO-2 include public availability and less than $1 \%$ contamination. Additionally, there was no significant difference in larval performance when larvae were reared on WCRMO-2 and the best proprietary diet. The new improvements accomplish the goal of formulating a diet comprised of available ingredients that supports performance of WCR larvae equal to or better than that of publicly available formulations.
\end{abstract}




\section{Introduction}

The western corn rootworm (WCR), Diabrotica virgifera virgifera LeConte, is an economic pest of maize (Zea mays L.) in North America and parts of Europe. The impact of corn rootworm has been estimated at approximately $\$ 2$ billion annually (Mitchell 2011). Yield reduction by this pest primarily results from heavy larval feeding on maize root (Spike and Tollefson 1989,1991$)$ that causes detrimental effects on nutrient and water uptake (Kahler et al. 1985), facilitation of pathogen infestation (Palmer and Kommedahl 1969, Kurtz et al. 2010), and unharvested grain due to lodging (Spike and Tollefson 1988, 1989, 1991). A variety of management tactics (e.g. crop rotation, chemical insecticides, transgenic maize expressing insecticidal proteins from Bacillus thuringiensis (Bt) Berliner) have faltered due to adaptation of WCR populations (Ball and Weekman 1963, Meinke et al. 1998, Levine et al. 2002, Gray et al. 2009, Gassmann et al. 2011, Pereira et al. 2015, Zukoff et al. 2016, Ludwick et al. 2017). Insect resistance management (IRM) plans for western corn rootworm have been directed toward monitoring the development of resistance to each of the Bt products (EPA 2016). Diet bioassays can be a key component of IRM plans. However, an ideal diet (Lapointe et al. 2010b) used in diet bioassays should be publicly available, compatible with insecticide toxins, easy to use, low in contamination, and supporting larval development that is similar to larval feeding on corn roots.

We previously developed an improved diet for WCR larvae, hereafter referred to as "WCRMO-1" (Huynh et al. 2017), that was an optimization of the ingredients in a previously published diet for WCR. Pleau et al. (2002) developed the first WCR diet, which was modified from a diet for southern corn rootworm (SCR), Diabrotica undecimpunctata howardi Barber (Sutter et al. 1971, Rose and McCabe 1973, Marrone et al. 1985), by adding corn root powder, 
removing formalin, optimizing the $\mathrm{pH}$ and changing the concentration of wheat germ and linseed oils. These modifications resulted in a diet that doubled larval weight gain compared to larvae reared on the SCR formulation (Pleau et al. 2002). We improved the Pleau et al. diet by optimizing the concentration of several ingredients (i.e., agar, casein, cellulose, corn root powder, linseed oil, and sucrose) and adding wheat germ oil (Huynh et al. 2017). This resulted in a formulation that supported approximately $99 \%$ of larvae for survival and molting and further doubled the weight gain after 11 days compared with larvae reared on the Pleau et al. diet. Additionally, this formulation is publicly available, compatible with each of the four marketed Bt toxins (Ludwick et al. 2018) and had less than 1\% contamination (Huynh et al. 2017) through clean laboratory practices.

Mixture designs combined with response surface modeling have been highly efficient for use in diet improvement for WCR and another coleopteran species (Lapointe et al. 2008, Lapointe et al. 2010a, Huynh et al. 2017). Since insect diets are a mixture of several ingredients, a change in the number of ingredients and in the proportion of any ingredient results in a change in the relative composition of all ingredients. Mixture experiments are commonly designed based on Scheffé polynomials (Scheffé 1958) wherein the proportions of mixture components are varied and total amount of the mixture remains constant. Later, Piepel and Cornell (1987) developed a mixture-amount experiment expressing the parameters of the Scheffé model while varying the total amount of the mixture. The application of mixture experiments in combination with response surface modeling allows concurrently varying multiple ingredients to identify and characterize key components and predict an optimum formulation that maximizes all desired developmental traits (Cornell 2002, Lapointe et al. 2010b, Lapointe et al. 2010a, Myers et al. 2016, Huynh et al. 2017). 
Previous diet work for WCR highlighted an important role of corn root powder as a key component that had positive effects on WCR development. Exclusion of this ingredient resulted in a slower development of WCR (Pleau et al. 2002, Huynh et al. 2017). Corn root powder is the only ingredient that is not available for commercial purchase, limiting its use. To develop an ideal diet for WCR, this study focused on the development of a WCR diet with improved larval development and without corn root powder, making the WCR diet more available for researchers. By applying mixture designs combined with response surface modeling, we explored eight different protein ingredients derived from animal, plant, and yeast sources to identify key proteins and then maximized the key proteins based on integrated evaluation of life history parameters (weight, molting, and survival) while limiting contamination.

\section{Materials and methods}

\section{Insects}

Eggs of WCR (primary diapausing strain) were provided by the USDA-ARS laboratory in Brooking, SD. The eggs were incubated in Petri dishes containing 70 mesh sievedsoil in an incubator at $25^{\circ} \mathrm{C}$ in complete darkness. When approximately $5 \%$ of the eggs hatched, the eggs were washed out of the soils using a 60 mesh sieve (Hogentogler \& Co. Inc., Columbia, MD) with $25^{\circ} \mathrm{C}$ water. The remaining eggs were surface-treated based on descriptions by Pleau et al. (2002). First, the eggs were submerged in undiluted lysol ${ }^{\circledR}$ (Reckitt Benckiser, LLC, Parsippany, NJ) for 3 minutes and then the supernatant was removed and the eggs were triple rinsed with distilled water. Next, the eggs were submerged in 10\% formalin (HT501128, Sigma Aldrich, St. Louis, MO) for 3 minutes, and then triple rinsed with distilled water after removing 
the supernatant again. Finally, the eggs were dispensed onto a coffee filter paper (Pure Brew, Rockline Industries, Sheboygan, WI) placed inside a 16 oz. cup (LG8RB-0090, Solo Cup Company, Lake Forest, IL) using a $1 \mathrm{ml}$ disposable pipette (13-711-9a, Fisher Scientific, Pittsburg, PA). Several holes made in a lid (DM16R-0090, Solo Cup Company) of the container for ventilation with a number zero insect pin. The eggs were then incubated at $25^{\circ} \mathrm{C}$ in darkness. Neonate larvae that hatched in less than 24 hours were used for the insect bioassay.

\section{Diet preparation}

A proprietary diet provided by industry was used within a week of receipt. Other diets were poured using a procedure described in Huynh et al. (2017). Agar (A7002, Sigma-Aldrich) and distilled water were added to a $400 \mathrm{ml}$ glass beaker and the solution was boiled for 2 minutes using a microwave. The molten agar solution was poured into a blender (Hamilton Beach, Inc., Model 51101BZ) placed in a biological safety cabinet (Nuaire, Biological safety cabinet). Next, dry diet ingredients, i.e. corn gluten meal (49760, Sigma-Aldrich), cottonseed meal (Down-ToEarth, Eugene, OR), plant protein (Perfect supplements, Coventry, RI), whey protein (ON, Downers Grove, IL), perfect amino (Bodyhealth, Clearwater, FL), yeast extract (BP1422, Fisher Scientific, Fair Lawn, NJ), egg powder (Judee's gluten free, Columbus, OH), casein (1100, BioServ), wheat germ (1661, Bio-Serv, Flemington, NJ), cellulose (3425, Bio-Serv), glucose (D16, Fisher Scientific), sucrose (04821721, MP Biomedicals, Santa Ana, CA), corn root powder (USDA-ARS, Columbia, MO), salt mix (F8680, Bio-Serv), vitamin mix (V1007, SigmaAldrich), methyl paraben (H5501, Sigma-Aldrich), cholesterol (C8503, Sigma-Aldrich), and sorbic acid (S1626, Sigma-Aldrich) were added (if applicable) to the blender and mixed thoroughly at low speed. The $\mathrm{pH}$ of the diet monitored by indicator strips (Whatman ${ }^{\circledR}$ 09-876- 
18, GE Healthcare Bio-Sciences, Pittsburg, PA) was increased to a $\mathrm{pH}$ of 9.0 by adding $10 \%$ $\mathrm{KOH}(\mathrm{w} / \mathrm{v})(\mathrm{P} 250$, Fisher Scientific). Next, the diet was poured in to a $750 \mathrm{ml}$ glass beaker placed on hot plate (Thermo scientific, Cimarec ${ }^{\mathrm{TM}}$ ) at $65^{\circ} \mathrm{C}$. A 96-well plate (3370, Corning Inc., Corning, NY) was used to accommodate the diet mixture with each well receiving $200 \mu$ l of the diet using a repeater pipette (Eppendorf repeater plus). The plate was opened to evaporate excess moisture for 15 minutes in the biological safety cabinet and then stored in a refrigerator at $4^{\circ} \mathrm{C}$. Diets were used within a week of being made for diet bioassays.

\section{Insect artificial diet bioassays}

The diet bioassays were conducted as described by in Huynh et al. (2017). All materials used in the diet assays were surface-treated via exposure to UV light for $10 \mathrm{~min}$ in biological cabinet. Each formulation was randomly assigned to a 12-well row of the 96-well plate and replicated 5 times for a total of 60 larvae per each formulation. Each well had one larva infested using a fine paintbrush. The plate was covered with a sealing film (TSS-RTQ-100, Excel Scientific, Inc., Victorville, CA). For ventilation, a hole was made with a number zero insect pin in the sealing film over each well. The plates were kept an incubator at $25^{\circ} \mathrm{C}$ in darkness for 10 days. Larval weight, molting, survival, and evidence of diet contamination were recorded at 10 days. For larval dry weight, all live larvae were pooled per replicate (12 possible) into $95 \%$ ethanol, dried in an oven (602752, Blue M Therm Dry Bacteriological Incubator) at $50^{\circ} \mathrm{C}$ for 2 days, and weighed using a micro balance (MSU6.6S-000-DM, Sartorius Lab Instruments GmbH \& Co. KG, Goettingen, Germany). 


\section{Eight-protein screening design for substitutes for corn root powder}

Previous work by Pleau et al. (2002) and Huynh et al. (2017) illustrated that protein components were key ingredients that had positive effects on overall WCR larval performance. To eliminate corn root powder, we explored contributions of eight different protein sources (i.e., corn gluten meal, cottonseed, casein, egg powder, plant protein, perfect amino, yeast extract, and whey protein) to three life history parameters (weight, molt, and survival) by constructing an eight-component I-optimal mixture design sufficient to satisfy a Scheffé quadratic polynomial response surface models (Cornell 2002). I-optimal mixture designs refer to mathematical algorithm that identifies diet blends that predict precise responses by minimizing the average variance of prediction across the design space (Laake 1975, Goos et al. 2016). Additional points to estimate lack of fit, which measures how well the model is fitted by the data, and replicated points to obtain sufficient degree of freedom for estimating pure error and to attain a uniform leverage for design spaces (Weisberg 1985) were also generated. This design had 30 design points with 7 model, 17 lack of fit and 5 pure error degrees of freedom (Table 9). Concentrations of other ingredients were kept at the level of WCRMO-1 (Table 10), except for an elimination of corn root powder, a substitution of sucrose by glucose, which is a major component of simple sugars in corn roots (Bernklau and Bjostad 2008), and a removal of oil components (linseed oils and wheat germ oils) because of their minor contributions to WCR diet (Huynh et al. 2017).

\section{Three-protein mixture-amount design for characterizing key protein sources}

Based on the results of the first experiment, three proteins that had the greatest effects on WCR larval performance (weight, molt, survival) were used to construct a D-optimal mixture-amount design that is sufficient for a Scheffé quadratic-quadratic polynomial response 
surface model (Cornell 2002). D-optimal mixture designs refer to the mathematical algorithm that chooses diet blends that focus on building precise model estimation (Czitrom 1988, Goos et al. 2016) by maximizing the determinant of the information matrix, and are appropriate for experimental designs that combine mixture and process variables (Eriksson et al. 2000). A mixture-amount experiment is a type of mixture experiment that varies both mixture component proportions and the total amount of the mixture (Cornell 2002). In this experiment, a threecomponent mixture design was generated with Design-Expert (Stat-Ease, Inc., Minneapolis, $\mathrm{MN}$ ) to characterize the effects of varying three animal proteins (casein, egg powder, and whey protein). These proteins are responsible for the largest effects on all developmental traits and were varied in blends from 1 gram to 6 grams. This design consisted of 32 design points with 17 model, 6 lack of fit and 8 pure error degrees of freedom (Table 11). Additional design points and duplicated points were included in order to satisfy the model terms, estimate lack of fit, attain sufficient degrees of freedom for estimating pure error, and to attain a uniform leverage for design spaces (Weisberg 1985). Other ingredients were kept constant as shown in Table 10.

\section{Egg powder experiment to identify optimum formulation}

The mixture-amount experiment revealed that egg powder alone had largest effect on larval performance. An experiment to find an optimum proportion of egg powder was conducted by varying proportions of egg powder (i.e., $0 \%, 1 \%, 2 \%, 3 \%, 4 \%, 6 \%$, and 10\% w/w). Additionally, formulations containing $3 \%$ egg powder with additions of lipid components (linseed oils and wheat germ oils) and lipid components plus corn root powder were tested. The amount of lipid components and corn root powder added were the same as in WCRMO-1 diet (Huynh et al. 2017). The proportions of water were changed to incorporate changes in egg powder proportions and additions of lipid components and corn root powder. Other ingredients 
were kept constant (Table 10). Two WCR diets including WCRMO-1 (Huynh et al. 2017) and the current best proprietary diet were also included as positive controls.

\section{Statistical analyses}

The percentage of larvae surviving and molting per replicate were generated by dividing the number of larvae surviving and molting by the number of larvae initially infested and multiplying by 100 . Weight per larva per replicate was generated by dividing the dry weight per replicate by the number of larvae that survived.

In the eight-protein mixture and three-protein mixture-amount experiments, the best fit model for each measured response (weight, molt, and survival) was selected from all possible models from linear to quartic polynomials generated with Design Expert ${ }^{\circledR}$ (Stat-Ease, Inc., Minneapolis, MN). Model selection was based on several criteria including low model $P$-value, lack of fit $P$-value, low standard deviation, high R-values (R-squared, adjusted R-squared, and predicted R-squared) (Lapointe et al. 2008), close agreement between adjusted R-squared and predicted R-squared, and a low PRESS value (Allen 1971, Myers and Montgomery 2002). Once more than one satisfactory model was generated, adequacy tests were performed to further evaluate the selected model as described by Anderson and Whitcomb (Anderson and Whitcomb 2004, Anderson and Whitcomb 2007).

In the egg powder experiment, all response measures (weight, molt, and survival) were analyzed as a randomized complete block design using PROC MIXED in SAS (SAS 2013). 


\section{Results}

\section{Eight-protein screening experiment}

The eight-protein mixture experiment produced significant response surface models for all three measures of life history parameters including weight $\left(p<0.0001, F_{9}=42.26\right)$ with insignificant lack of fit (LOF) $(p=0.5382)$, molting $\left(p<0.0001, F_{11}=197.99\right)$ with significant LOF $(p=0.0397)$, and survival $\left(p<0.0001, F_{9}=50.80\right)$ with significant LOF $(p=0.0009)$ by varying 8 different protein sources: corn gluten meal, cottonseed meal, casein, plant protein, whey protein, perfect amino, yeast extract, and egg powder (Table 12). Pure errors of models for molt $($ sum of square $(\mathrm{SS})$ of pure error $=0.013)$ and survival $(\mathrm{SS}$ of pure error $=0.0003)$ were very small, and resulted in significant LOFs. All models had R-squared, predicted R-squared and adjusted R-squared values in reasonable agreement, i.e. the differences between the predicted and adjusted R-squared values were less than 0.2 . The relationships between protein sources and larval performance were shown in trace plots that determine the effects of changeable proportions of one component in relation to a reference blend while all the other components are held constant (Fig. 9, (Cornell 2002, Smith 2005)). The direction and magnitude of influence of the individual components on the measured response variables are indicated by the slope of the line.

All response models revealed that the three components responsible for the greatest effects on all overall larval developmental traits (weight, molt, and survival) were three animal protein sources, i.e. casein, egg powder, whey protein (Figs. 9A-C). For plant protein sources, plant protein and cottonseed meal, to some extent, had positive effects on all three criteria of larval performance whereas corn gluten meal and perfect amino had negative effects on all 
response measures. Yeast extract had minor positive effects on all larval performance compared to other positive components tested.

\section{Three-protein mixture-amount experiment}

The three-protein mixture-amount (casein, egg powder, and whey protein) yielded significant response surface models for all three measures, including larval weight $\left(p<0.0001, F_{4}\right.$ $=12.48)$ with significant LOF $(p<0.0001)$, molting $\left(p=0.0006, F_{5}=6.44\right)$ with significant LOF ( $p=0.0011)$, and survival $\left(p<0.0001, F_{6}=8.79\right)$ with insignificant LOF $(p=0.0584)$ (Table 13).

Models for weight and molt had significant LOFs due to very small values of pure error. Sums of square of pure error for models for weight and molt were 0.0013 and 0.0110 , respectively. The $\mathrm{R}$-squared, predicted R-squared and adjusted R-squared values of all models were clustered in the reasonable agreement. Ternary plots showed the relationships between protein sources, mixture amount and larval performance by estimating the influences of all possible combinations of mixture components on measured variables (Figs. $10 \& 12$ ). Color and labelled isobars indicated the magnitude of the response variables in a dimension that can be envisioned as perpendicular to the page.

All response models indicated that egg powder was the most important component to maximize all development traits (Figs. 10A-C). High proportions of egg powder resulted in an increase in all measures of larval performance whereas casein at high proportions resulted in a decrease in all response measures, especially in survival. An increase in the proportion of whey protein resulted in an increase in survival, but whey protein at high proportions had negative effects on weight and molting. There was a synergy blending effect of egg powder and whey 
protein on larval molting, indicating that an increase in egg powder with decreasing proportions of whey protein resulted in an increase in molting (Fig. 11).

The contour plots showed interactive effects between egg powder and whey protein and egg powder and casein in relation to varying total amount mixture while proportions of other components (casein or whey protein) were held constant (Figs. 12A-C). The results showed that egg powder at high proportions with higher total amount of mixtures could yield better larval weight molting, and survival. Since egg powder at high proportions had over $95 \%$ survival, the minor negative effect of increasing the proportion of egg powder at higher total amount of diet on survival was not considered.

\section{Egg powder optimization}

Increases in egg powder up to a proportion of $4 \%(\mathrm{w} / \mathrm{w})$ and $2 \%(\mathrm{w} / \mathrm{w})$ resulted in positive larval weight gain (Fig. 13A) and molting (Fig. 13B), respectively whereas additions of egg powder had no significant effect on survival (Fig. 13C). All diets tested had larval survivorship to $10 \mathrm{~d}$ that were higher than $95 \%$ and there were no significant differences in survival. Additions of oil components alone and oil components plus corn root powder to the diet did not yield a better formulation for any developmental traits (Figs. 13A-C).

The egg powder optimization produced a superior formulation, referred to hereafter as WCRMO-2 (Table 14). Compared with previous published WCR diets (Pleau et al. 2002, Huynh et al. 2017), WCRMO-2 had no corn root powder, additions of egg powder and glucose, and removals of oil components, casein and sucrose. At 10 days post infestation, larval dry weight increased 4 fold with WCRMO-2 as compared to WCRMO-1 and survival and molting rate of WCR larvae reared on WCRMO-2 were approximately 97\%. Additionally, there was no 
significant difference in all developmental traits when WCR larvae were reared on WCRMO-2 or the current best proprietary diet (unpublished data) at 10 days post infestation (Figs. $14 \& 15$ ).

\section{Contamination}

The contamination of all experiments was less than $1 \%$, a relationship between contamination and diet ingredients did not exist.

\section{Discussion}

Current published WCR diets require corn root powder as a key component that has a strong positive influence on larval performance (Pleau et al. 2002, Huynh et al. 2017). However, corn root powder is the only ingredient that is not currently commercially available and makes the WCR diet unavailable for many users. We applied response surface methodology combined with n-dimensional mixture designs to develop an improved diet for WCR without corn root powder by systematically exploring the effects of different protein sources on WCR based on life history parameters (weight, molt, and survival). By using geometric and mathematical approaches, we evaluated the influence of eight different protein sources to identify key protein sources and characterized and maximized benefits of the key protein, resulting in an improved formulation that will be more accessible to users.

The eight-protein mixture experiment indicated that animal protein sources supported all larval developmental traits (weigh, molt, survival) better than other protein sources such as plant and yeast proteins (Fig. 9). All three animal proteins had the greatest positive effects on overall larval performance. Increasing proportions of three animal proteins (casein, egg powder, and whey protein) resulted in higher improvements in overall response measures compared with other tested proteins. For plant protein sources, additions of plant protein and cottonseed meal 
improved all measured responses, but increases in the proportions of corn gluten meal and perfect amino had detrimental effects on the three measured responses. Compared with other proteins tested, yeast protein had the smallest effects on all developmental traits. Pleau et al. (2002) reported a similar pattern in which WCR larvae performed better with proteins (casein and sodium caseinate) derived from animals compared with proteins (lima bean and pinto beans) derived from plants.

The three-protein mixture-amount experiment to characterize the key protein components revealed that egg powder was the best protein source and egg powder alone at higher amount of diet blends could yield the best formulation based on all developmental traits (weight, molt, and survival) compared to possible combinations of three animal proteins, i.e. casein, egg powder, whey protein (Figs. $10 \& 12$ ). There was a nonlinear synergy blending effect between egg powder and whey protein on molting (Fig. 11) and this interaction was important when egg powder was at low proportions (Fig. 12C). Casein at high proportions had a negative effect on survival and molting. The similar pattern of the negative effects of casein on larval survival and molting was reported in previous work of WCR diets (Pleau et al. 2002, Huynh et al. 2017).

The application of mixture experiments with response surface modeling has shown value in insect diet improvement by identifying and maximizing key ingredients in existing coleopteran diets (Lapointe et al. 2008, Lapointe et al. 2010b, Lapointe et al. 2010a, Huynh et al. 2017). By using this approach, we characterized eight different protein ingredients from animal, plant and yeast sources and maximized the key protein component based on all larval response measures. This resulted in a superior formulation (WCRMO-2) with the addition of egg powder, removal of corn root powder, removal of lipid components (linseed oils and wheat germ oils) and 
a substitution of sucrose with glucose compared with WCRMO-1 (Huynh et al. 2017). At 10 days post infestation, the level of diet improvement included a 4-fold increase in larval weight gain with WCRMO-2 as compared to WCRMO-1 whereas survival and molting rate of WCR larvae on WCRMO-2 at approximately 97\%. There was no significant difference on overall larval performance when WCR larvae were reared on WCRMO-2 compared with the current superior proprietary diet (Fig. 14). Additionally, WCRMO-2 without corn root powder had basically no contamination. The new formulation significantly improved larval performance and is more available for users. This is a significant accomplishment toward the long-term goal to develop the ideal diet that is publicly available, easy to use, and produces insects that are physically similar to WCR larvae fed on corn roots. Further efforts to improve artificial diets for continuous rearing WCR that focus on adult emergence, and fecundity in the next generation would be beneficial. 
Table 9. Diet blends of 8 proteins (gram) varied in a mixture screening design to rear western corn rootworm larvae

\begin{tabular}{crrrrrrrr}
\hline Diet blend \# & $\begin{array}{r}\text { Corn gluten } \\
\text { meal }\end{array}$ & $\begin{array}{r}\text { Cottonseed } \\
\text { meal }\end{array}$ & Casein & $\begin{array}{r}\text { Egg } \\
\text { powder }\end{array}$ & $\begin{array}{r}\text { Plant } \\
\text { protein }\end{array}$ & $\begin{array}{r}\text { Perfect } \\
\text { Amino }\end{array}$ & $\begin{array}{r}\text { Yeast } \\
\text { extract }\end{array}$ & $\begin{array}{r}\text { Whey } \\
\text { protein }\end{array}$ \\
\hline 1 & 0.38 & 0.38 & 0.38 & 0.38 & 0.37 & 0.37 & 0.37 & 0.37 \\
2 & 0.43 & 0 & 0.43 & 0.43 & 0.43 & 0.43 & 0.43 & 0.42 \\
3 & 0 & 0.43 & 0.43 & 0.43 & 0.43 & 0.43 & 0.43 & 0.42 \\
4 & 0.43 & 0.43 & 0.43 & 0.43 & 0 & 0.43 & 0.43 & 0.42 \\
5 & 0.43 & 0.43 & 0.43 & 0 & 0.43 & 0.43 & 0.43 & 0.42 \\
6 & 0.38 & 0.38 & 0.38 & 0.38 & 0.37 & 0.37 & 0.37 & 0.37 \\
7 & 0 & 0 & 0 & 0 & 0 & 0 & 3 & 0 \\
8 & 0.43 & 0.43 & 0.43 & 0.43 & 0.43 & 0 & 0.43 & 0.42 \\
9 & 0.38 & 0.38 & 0.38 & 0.38 & 0.37 & 0.37 & 0.37 & 0.37 \\
10 & 3 & 0 & 0 & 0 & 0 & 0 & 0 & 0 \\
11 & 0.38 & 0.38 & 0.38 & 0.38 & 0.37 & 0.37 & 0.37 & 0.37 \\
12 & 0.19 & 0.19 & 0.19 & 0.19 & 0.19 & 1.67 & 0.19 & 0.19 \\
13 & 0 & 0 & 0 & 3 & 0 & 0 & 0 & 0 \\
14 & 0.19 & 0.19 & 0.19 & 1.67 & 0.19 & 0.19 & 0.19 & 0.19 \\
15 & 0.43 & 0.43 & 0 & 0.43 & 0.43 & 0.43 & 0.43 & 0.42 \\
16 & 1.67 & 0.19 & 0.19 & 0.19 & 0.19 & 0.19 & 0.19 & 0.19 \\
17 & 0 & 0 & 3 & 0 & 0 & 0 & 0 & 0 \\
18 & 0.19 & 0.19 & 1.67 & 0.19 & 0.19 & 0.19 & 0.19 & 0.19 \\
19 & 0 & 0 & 0 & 0 & 0 & 0 & 0 & 3 \\
20 & 0 & 0 & 0 & 0 & 0 & 3 & 0 & 0 \\
21 & 0.19 & 1.67 & 0.19 & 0.19 & 0.19 & 0.19 & 0.19 & 0.19 \\
22 & 0.19 & 0.19 & 0.19 & 0.19 & 0.19 & 0.19 & 0.19 & 1.67 \\
23 & 0.43 & 0.43 & 0.43 & 0.43 & 0.43 & 0.43 & 0 & 0.42 \\
24 & 0 & 0 & 0 & 0 & 3 & 0 & 0 & 0 \\
25 & 0.19 & 0.19 & 0.19 & 0.19 & 1.67 & 0.19 & 0.19 & 0.19 \\
26 & 0.19 & 0.19 & 0.19 & 0.19 & 0.19 & 0.19 & 1.67 & 0.19 \\
27 & 0.38 & 0.38 & 0.38 & 0.38 & 0.37 & 0.37 & 0.37 & 0.37 \\
28 & 0 & 3 & 0 & 0 & 0 & 0 & 0 & 0 \\
29 & 0.43 & 0.43 & 0.43 & 0.43 & 0.43 & 0.43 & 0.42 & 0 \\
30 & 3 & 0 & 0 & 0 & 0 & 0 & 0 & 0 \\
\hline & & & & & & & &
\end{tabular}


Table 10. Components that were held constant in diets used to rear western corn rootworm larvae in the 8-protein mixture experiment.

\begin{tabular}{ll}
\multicolumn{1}{c}{ Components } & Amount \\
\hline 1. Agar & $1.5 \mathrm{~g}$ \\
2. Cellulose & $1.0 \mathrm{~g}$ \\
3. Chlortetracycline $(10 \mathrm{mg} / \mathrm{ml})$ & $6.4 \mathrm{mg}$ \\
4. Cholesterol & $6.0 \mathrm{mg}$ \\
5. Distilled water & $88 \mathrm{ml}$ \\
6. Food coloring & $6.4 \mathrm{mg}$ \\
7. Glucose & $1.0 \mathrm{~g}$ \\
8. Methyl paraben & $0.1 \mathrm{~g}$ \\
9. Potassium hydroxide $(10 \%)$ & $3.5 \mathrm{ml}$ \\
10. Sorbic acid & $6.4 \mathrm{mg}$ \\
11. Streptomycin $(12.8 \mathrm{mg} / \mathrm{ml})$ & $6.4 \mathrm{mg}$ \\
12. Vanderzant vitamin $\mathrm{mix}$ & $0.90 \mathrm{~g}$ \\
13. Wesson's salt mix & $0.93 \mathrm{~g}$ \\
14. Wheat germ, ground & $6.0 \mathrm{~g}$ \\
\hline
\end{tabular}


Table 11. Mixture-amount (concentration) design points to determine the optimum combination of key protein ingredients (gram)

\begin{tabular}{|c|c|c|c|c|}
\hline \multirow{2}{*}{ Diet blend \# } & \multicolumn{3}{|c|}{ Proportion of mixture components } & \multirow{2}{*}{$\begin{array}{c}\text { Total mixture amount } \\
{[\text { Casein }+ \text { Egg powder }+ \text { Whey protein }]}\end{array}$} \\
\hline & Casein & Egg powder & Whey protein & \\
\hline 1 & 0 & 0.5 & 0.5 & 6 \\
\hline 2 & 0.16 & 0.16 & 0.67 & 2.25 \\
\hline 3 & 0.16 & 0.67 & 0.16 & 2.25 \\
\hline 4 & 0.5 & 0 & 0.5 & 6 \\
\hline 5 & 1 & 0 & 0 & 3.5 \\
\hline 6 & 0.5 & 0.5 & 0 & 6 \\
\hline 7 & 1 & 0 & 0 & 6 \\
\hline 8 & 0 & 1 & 0 & 6 \\
\hline 9 & 1 & 0 & 0 & 1 \\
\hline 10 & 0.5 & 0 & 0.5 & 1 \\
\hline 11 & 0.67 & 0.16 & 0.16 & 4.75 \\
\hline 12 & 0.16 & 0.16 & 0.67 & 4.75 \\
\hline 13 & 0.5 & 0.5 & 0 & 1 \\
\hline 14 & 0 & 1 & 0 & 6 \\
\hline 15 & 0 & 1 & 0 & 3.5 \\
\hline 16 & 0 & 0.5 & 0.5 & 1 \\
\hline 17 & 1 & 0 & 0 & 1 \\
\hline 18 & 0 & 0.5 & 0.5 & 3.5 \\
\hline 19 & 0.5 & 0.5 & 0 & 3.5 \\
\hline 20 & 0.5 & 0.5 & 0 & 1 \\
\hline 21 & 0.5 & 0 & 0.5 & 3.5 \\
\hline 22 & 0 & 0 & 1 & 1 \\
\hline 23 & 0 & 0.5 & 0.5 & 1 \\
\hline 24 & 0 & 0 & 1 & 1 \\
\hline 25 & 0.16 & 0.67 & 0.16 & 4.75 \\
\hline 26 & 0 & 1 & 0 & 1 \\
\hline 27 & 0 & 0 & 1 & 3.5 \\
\hline 28 & 0 & 0 & 1 & 6 \\
\hline 29 & 1 & 0 & 0 & 6 \\
\hline 30 & 0 & 1 & 0 & 1 \\
\hline 31 & 0.67 & 0.16 & 0.16 & 2.25 \\
\hline 32 & 0 & 0 & 1 & 6 \\
\hline
\end{tabular}


Table 12. $p$-values, regression coefficients and response surface model fitting diagnostic statistics for WCR larval responses to 8-component diet mixtures

\begin{tabular}{|c|c|c|c|c|c|c|}
\hline & $\begin{array}{l}\text { Weight } \\
p \text {-values }\end{array}$ & $\begin{array}{l}\text { Regression } \\
\text { coefficients }^{\mathrm{a}}\end{array}$ & $\begin{array}{l}\% \text { Molt } \\
p \text {-values }\end{array}$ & $\begin{array}{l}\text { Regression } \\
\text { coefficients }\end{array}$ & $\begin{array}{l}\% \text { Survival } \\
p \text {-values }\end{array}$ & $\begin{array}{l}\text { Regression } \\
\text { coefficients }\end{array}$ \\
\hline Model & $<0.0001$ & - & $<0.0001$ & - & $<0.0001$ & - \\
\hline Linear mixture & $<0.0001$ & - & $<0.0001$ & - & $<0.0001$ & - \\
\hline A & - & 6.74 & - & -1.78 & - & 0.48 \\
\hline B & - & 3.62 & - & -0.31 & - & 0.93 \\
\hline $\mathrm{C}$ & - & 2.46 & - & -0.01 & - & 0.96 \\
\hline D & - & -0.54 & - & 1.02 & - & 0.98 \\
\hline $\mathrm{E}$ & - & 2.71 & - & -0.13 & - & 0.99 \\
\hline $\mathrm{F}$ & - & 6.88 & - & -1.86 & - & 1.05 \\
\hline G & - & 0.03 & - & 1.06 & - & 1.22 \\
\hline $\mathrm{H}$ & - & 1.24 & - & 0.03 & - & 1.00 \\
\hline $\mathrm{A}^{2}$ & - & - & 0.0082 & -1.26 & - & - \\
\hline $\mathrm{D}^{2}$ & 0.0151 & 3.89 & 0.0058 & -1.39 & - & - \\
\hline $\mathrm{F}^{2}$ & - & - & 0.0174 & -1.17 & 0.0026 & -0.48 \\
\hline $\mathrm{G}^{2}$ & 0.0102 & 4.15 & 0.0002 & -2.12 & 0.0073 & -0.42 \\
\hline Lack of fit & 0.5382 & & 0.0397 & & 0.0009 & \\
\hline Model type & $\begin{array}{l}\text { Quadratic } \\
\text { (reduced) }\end{array}$ & & $\begin{array}{l}\text { Quadratic } \\
\text { (reduced) }\end{array}$ & & $\begin{array}{l}\text { Quadratic } \\
\text { (reduced) }\end{array}$ & \\
\hline Transformation & $\begin{array}{l}\text { Inverse } \\
\text { square root }\end{array}$ & & Base $10 \log$ & & Square root & \\
\hline $\mathrm{R}^{2}$ & 0.9500 & & 0.9918 & & 0.9581 & \\
\hline $\mathrm{R}_{\text {adj }}^{2}$ & 0.9276 & & 0.9868 & & 0.9392 & \\
\hline $\mathrm{R}_{\text {pred }}^{2}$ & 0.8196 & & 0.9538 & & 0.8243 & \\
\hline
\end{tabular}

A: corn gluten meal, B: cottonseed meal, C: casein, D: plant protein, E: whey protein, F: perfect amino, G: yeast extract, $\mathrm{H}$ : egg powder, ${ }^{\mathrm{a}}$ Expressed in coded forms. Mixture component coding is L_Pseudo (Cornell 2002). 
Table 13. $p$-values, regression coefficients and response surface model fitting diagnostic statistics for WCR larval responses to 3-protein mixture-amount experiment

\begin{tabular}{|c|c|c|c|c|c|c|}
\hline & $\begin{array}{l}\text { Weight } \\
p \text {-values }\end{array}$ & $\begin{array}{l}\text { Regression } \\
\text { coefficients }^{\mathrm{a}}\end{array}$ & $\begin{array}{l}\% \text { Molt } \\
p \text {-values }\end{array}$ & $\begin{array}{l}\text { Regression } \\
\text { coefficients }\end{array}$ & $\begin{array}{l}\% \text { Survival } \\
p \text {-values }\end{array}$ & $\begin{array}{l}\text { Regression } \\
\text { coefficients }\end{array}$ \\
\hline Model & $<0.0001$ & - & 0.0006 & - & $<0.0001$ & - \\
\hline Linear mixture & $<0.0001$ & - & 0.0119 & - & $<0.0001$ & - \\
\hline A & - & 0.32 & - & 0.89 & - & 0.96 \\
\hline B & - & 0.14 & - & 0.70 & - & 1.00 \\
\hline $\mathrm{C}$ & - & 0.13 & - & 0.71 & - & 0.85 \\
\hline $\mathrm{A} \times \mathrm{B}$ & - & - & 0.0267 & 0.65 & - & - \\
\hline $\mathrm{A} \times \mathrm{C}$ & - & - & - & - & 0.0008 & 0.34 \\
\hline $\mathrm{B} \times \mathrm{C}$ & - & - & - & - & - & - \\
\hline AxConc. ${ }^{b}$ & 0.0001 & 0.13 & 0.0127 & 0.16 & $<0.0001$ & -0.09 \\
\hline $\mathrm{B} \times$ Conc. & 0.0048 & -0.08 & 0.0039 & -0.16 & - & - \\
\hline Lack of fit & $<0.0001$ & & 0.0011 & & 0.0584 & \\
\hline Model type & $\begin{array}{l}\text { Linear mixture } \\
\text { (reduced) } \mathrm{x} \\
\text { linear amount }\end{array}$ & & $\begin{array}{l}\text { Quadratic } \\
\text { mixture } \\
\text { (reduced) } x \\
\text { linear amount }\end{array}$ & & $\begin{array}{l}\text { Quadratic } \\
\text { mixture } \\
\text { (reduced) } \mathrm{x} \\
\text { linear amount }\end{array}$ & \\
\hline Transformation & N/A & & N/A & & N/A & \\
\hline $\mathrm{R}^{2}$ & 0.6575 & & 0.5629 & & 0.7816 & \\
\hline $\mathrm{R}_{\text {adj }}^{2}$ & 0.6048 & & 0.4754 & & 0.7480 & \\
\hline $\mathrm{R}_{\text {pred }}^{2 \mathrm{adj}}$ & 0.5200 & & 0.3775 & & 0.6597 & \\
\hline
\end{tabular}

A: egg powder, B: whey protein, C: casein. ${ }^{\text {a }}$ Mixture component coding is L_Pseudo (Cornell 2002), ${ }^{\mathrm{b}}$ Concentration 
Table 14. Artificial diets for WCR larvae.

\begin{tabular}{|c|c|c|c|}
\hline Ingredients & Pleau diet $^{a}$ & WCRMO-1 $^{\text {b }}$ & WCRMO-2 \\
\hline Egg powder & - & - & + \\
\hline Glucose & - & - & + \\
\hline Wheat germ (raw, ground) & + & + & + \\
\hline Cellulose & + & + & + \\
\hline Agar & + & + & + \\
\hline Casein & + & + & - \\
\hline Corn root powder & + & + & - \\
\hline Sucrose & + & + & - \\
\hline Linseed oil, raw & + & + & - \\
\hline Wheat germ oil & - & + & - \\
\hline Cholesterol & + & + & + \\
\hline Wesson's salt mix & + & + & + \\
\hline Vanderzant Vitamin mix & + & + & + \\
\hline Methyl paraben & + & + & + \\
\hline Sorbic acid & + & + & + \\
\hline Potassium hydroxide $(10 \%)$ & + & + & + \\
\hline Streptomycin $(12.8 \mathrm{mg} / \mathrm{ml})$ & + & + & + \\
\hline Chlortetracycline $(10.0 \mathrm{mg} / \mathrm{ml})$ & + & + & + \\
\hline Distilled water & + & + & + \\
\hline Green food coloring & + & + & + \\
\hline
\end{tabular}

${ }^{\mathrm{a}}$ Pleau et al. (2002), ${ }^{\mathrm{b}}$ Huynh et al. (2017) 

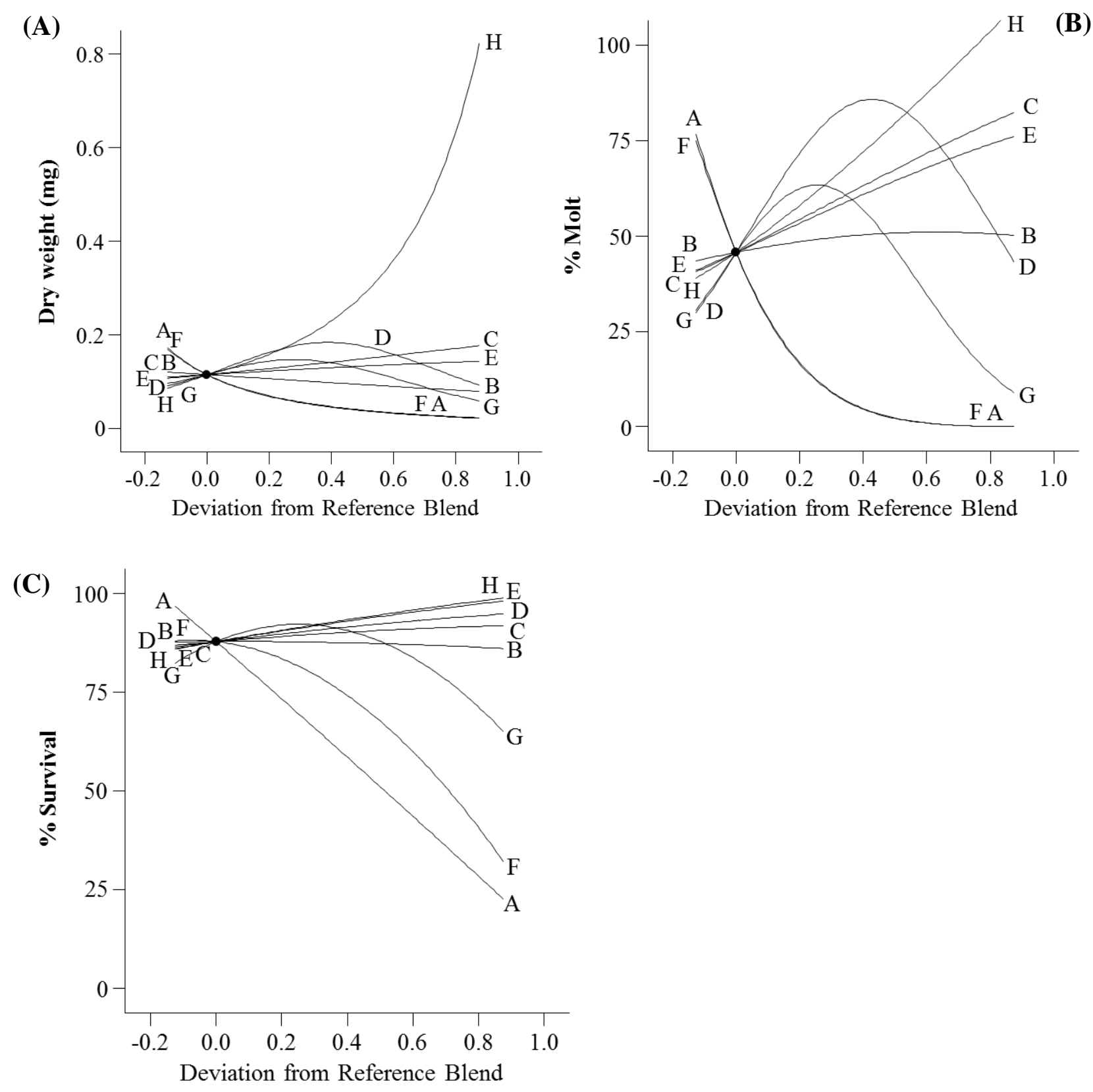

Figure 9. Trace plot of WCR larval responses deviation from a reference blend diet in 8-protein screening experiment. (A): weight, (B): molting, (C): survival. Reference blend proportions: corn gluten meal $=$ cottonseed meal $=$ casein $=$ plant protein $=$ whey protein $=$ perfect amino $=$ yeast extract $=$ egg powder $=0.375$. A: corn gluten meal, B: cottonseed meal, C: casein, D: plant protein, E: whey protein, F: perfect amino, G: yeast extract, H: egg powder. 

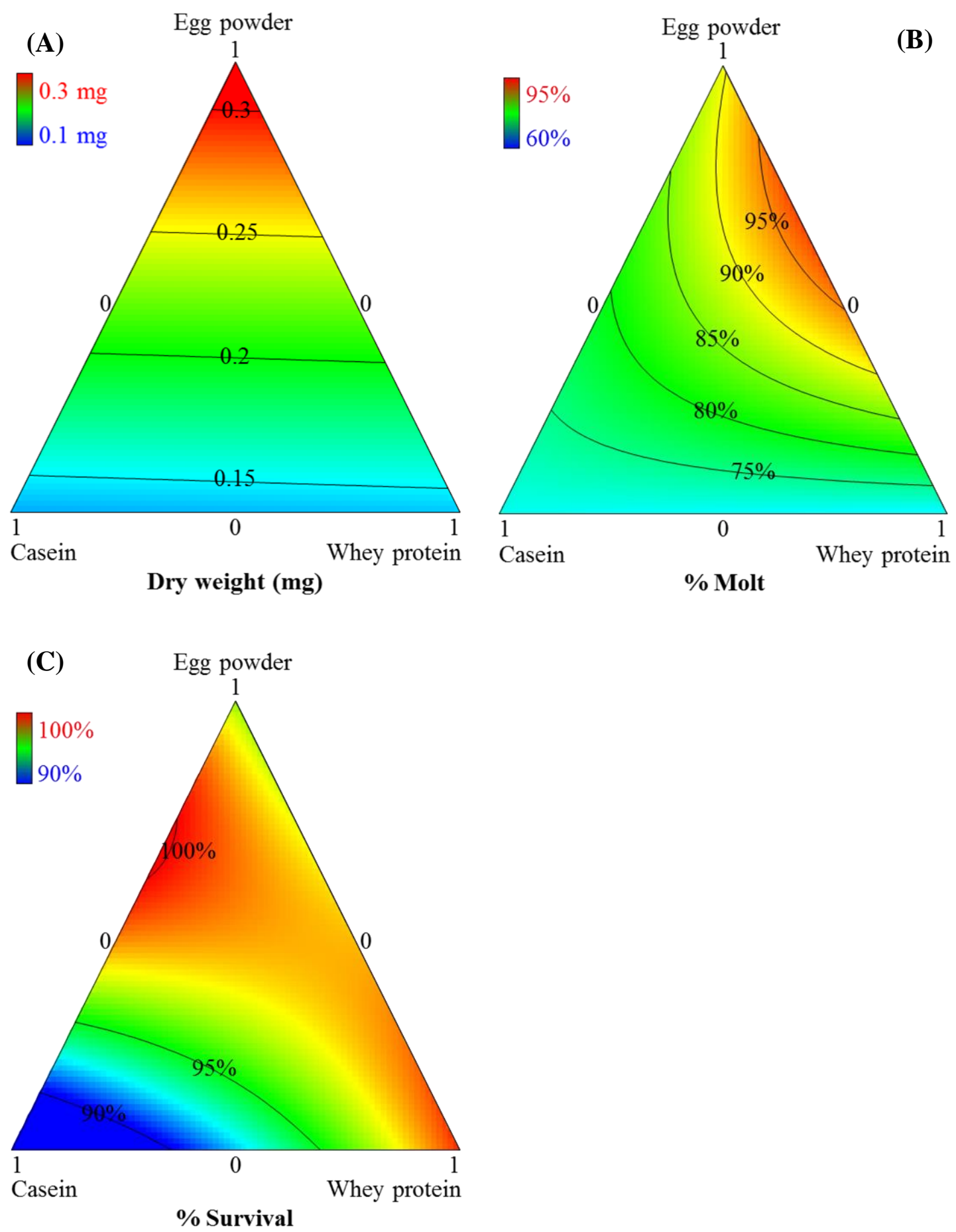

Figure 10. Contour plots of larval responses for WCR reared on different diets from mixtureamount design of casein:egg powder:whey protein at 10 days post infestation. (A) weight, (B) survival, and (C) molting. Color bars display the magnitude of the measured response. Total amount of mixture $=3$ grams. 


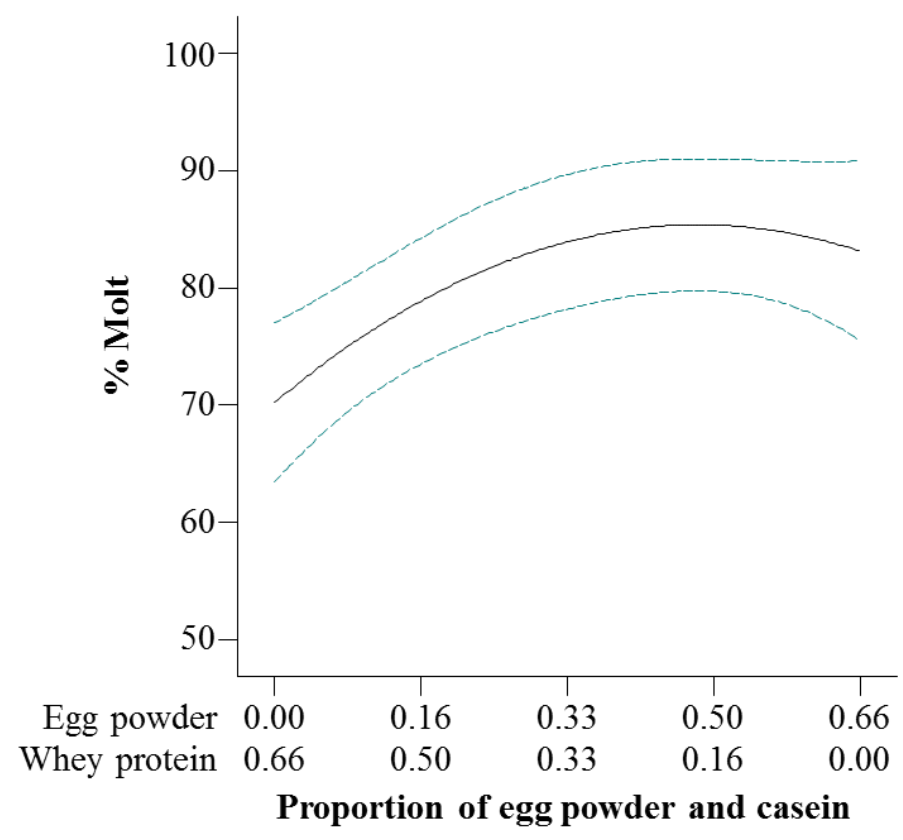

Figure 11. Nonlinear blending effects of egg powder $\times$ whey protein on molting from the mixture-amount experiment. Dotted lines indicate $95 \%$ confidence interval bands. Proportions of casein protein $=0.34$. Total amount of mixture $=3$ grams. 
(A)

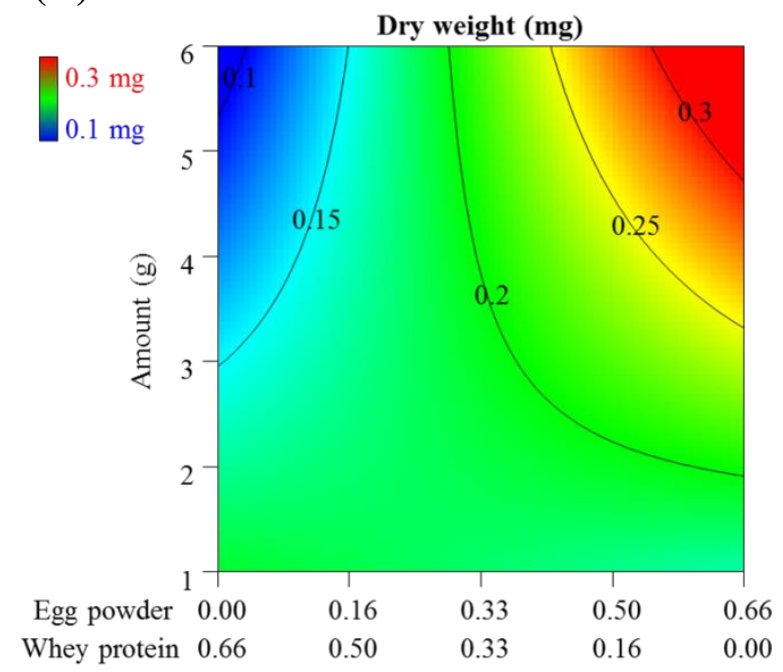

Proportion of egg powder and whey protein
(B)

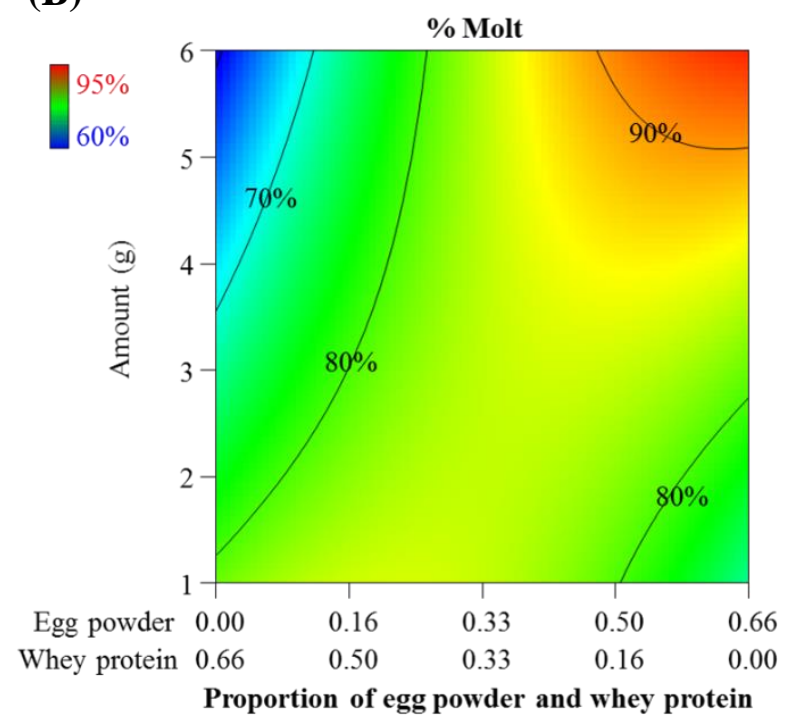

(C)

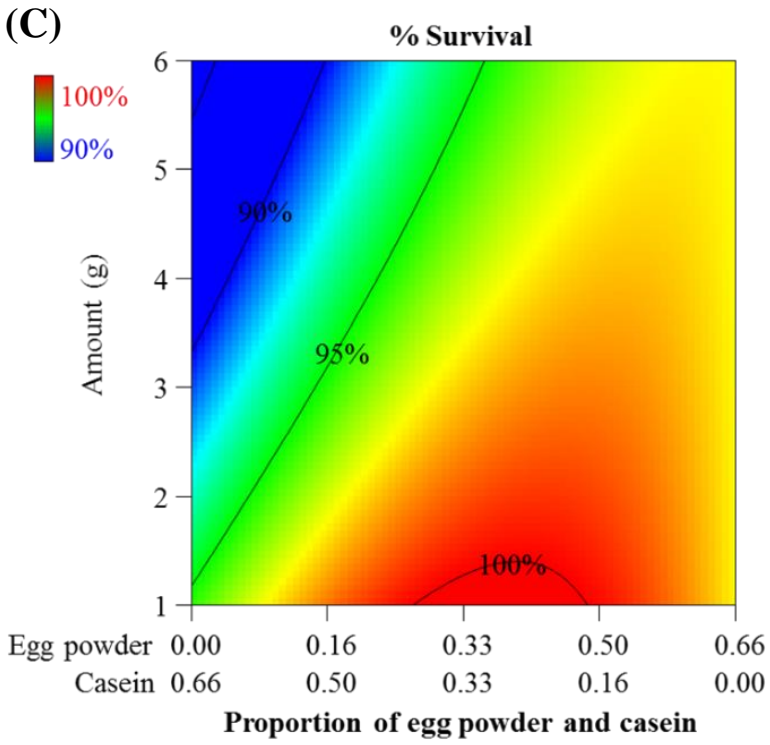

Figure 12. Contour plots of the interaction of egg powder, casein and concentration on WCR larvae reared on different diets from the mixture-amount design at 10 days post infestation. (A) weight, (B) survival, and (C) molting. (A \& B) Proportions of casein = 0.34; (C) Proportions of whey protein $=0.34$. Color bars display the magnitude of the measured response. 


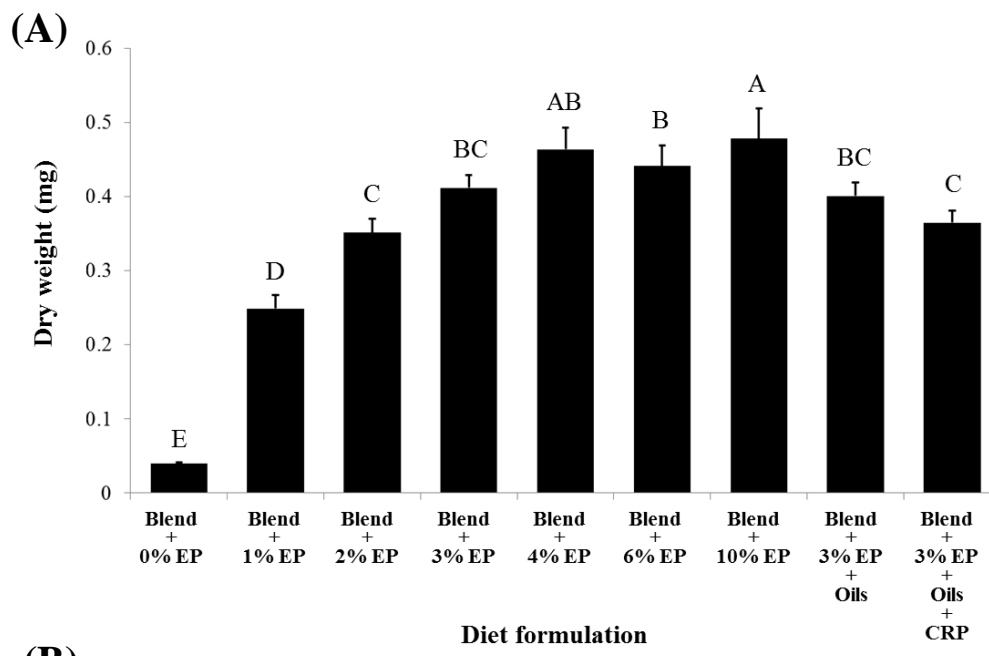

(B)
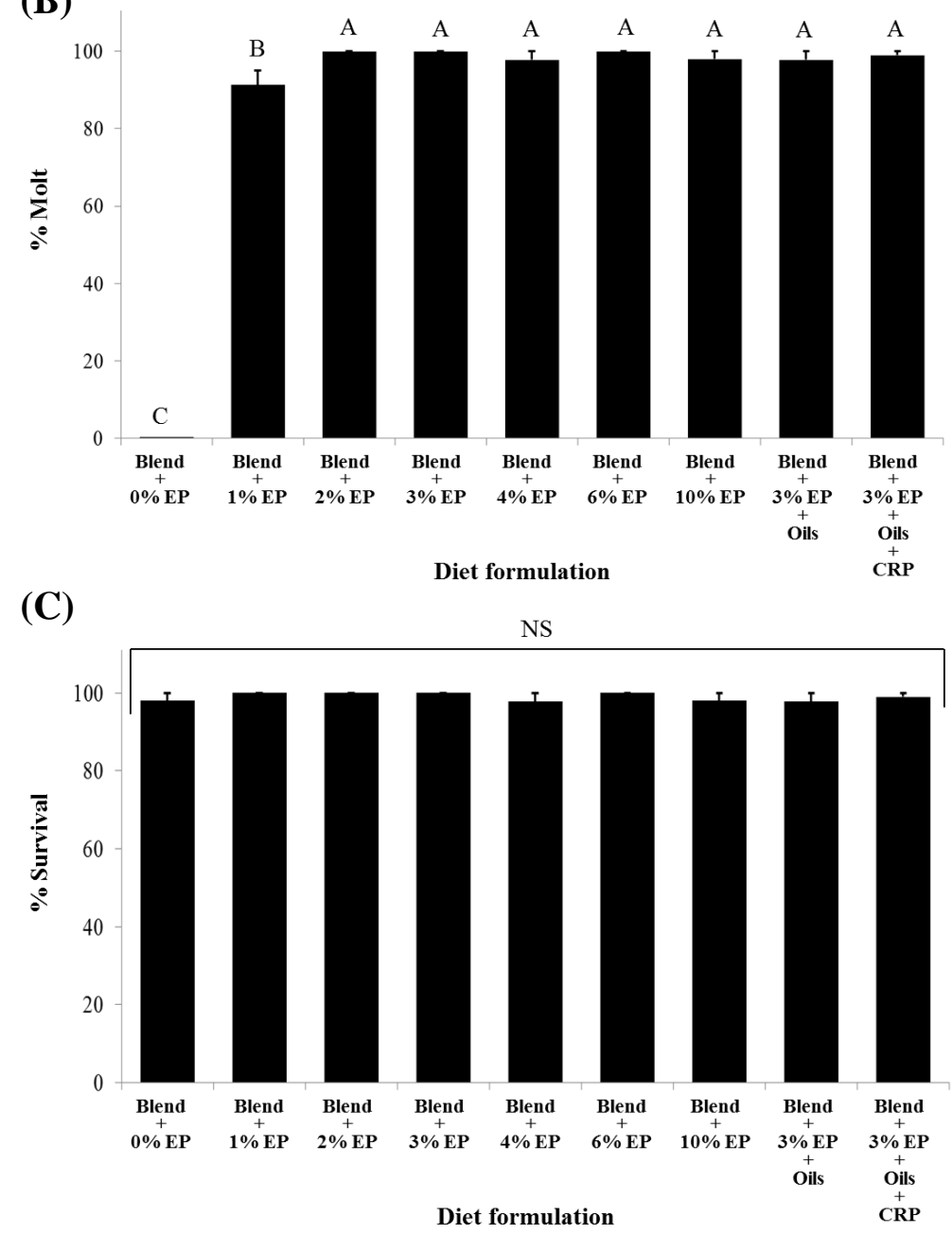

Figure 13. Larval dry weight, survival, and percent successful completion of molt for western corn rootworm larvae reared on different diets. (A) weight $\left(p<0.0001, F_{8,32}=32.95\right)$, (B) molting $\left(p<0.0001, F_{8,32}=97.73\right)$, and $(\mathrm{C})$ survival $\left(p=0.8155, F_{8,32}=0.54\right)$. EP: whole egg powder, oils: linseed oils and wheat germ oils, CRP: corn root power. Means with bars followed by different letters are significantly different $(p<0.05)$. Means \pm SEM 


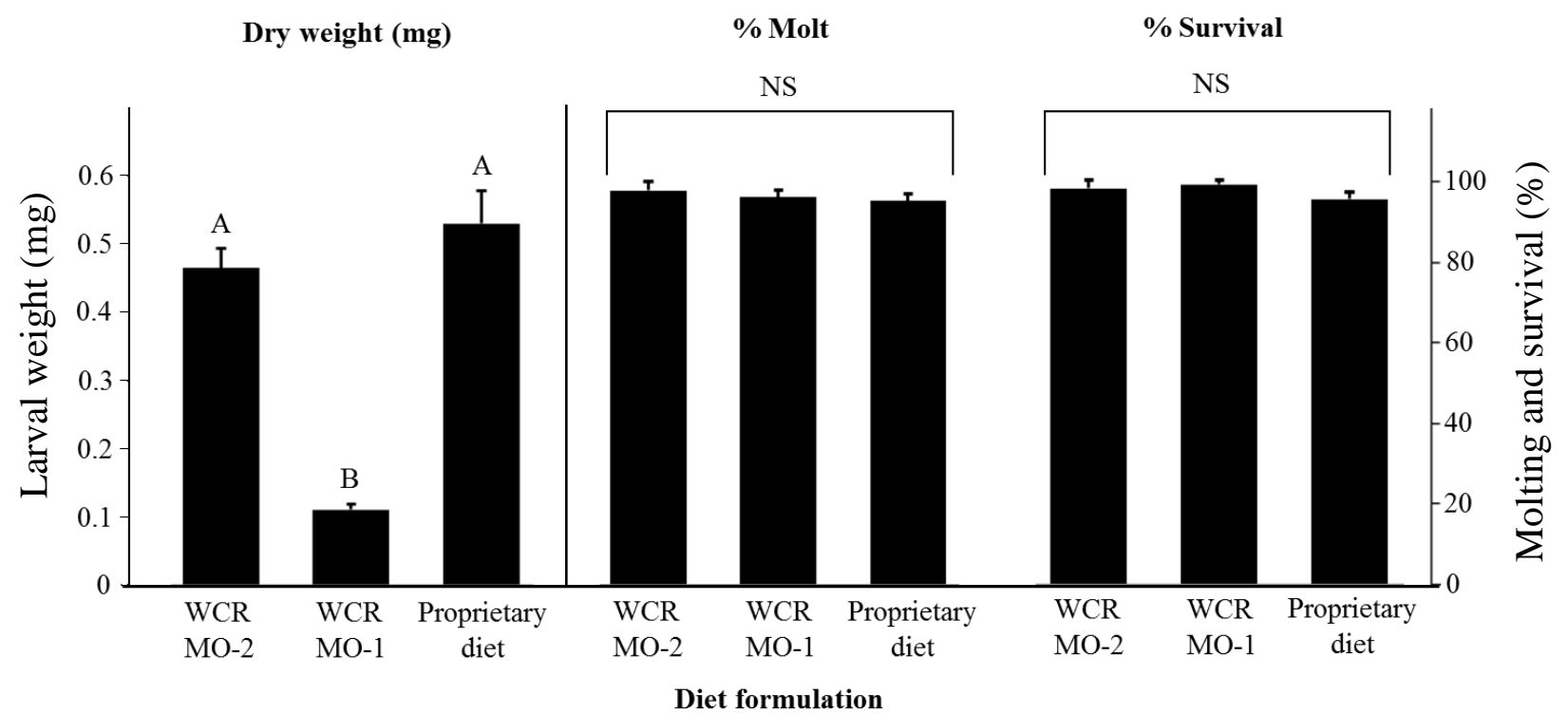

Figure 14. Larval dry weight, survival, and percent successful completion of molt for western corn rootworm larvae reared on WCRMO-2, WCRMO-1, and a proprietary diet for 10 days. Means with bars followed by different letters are significantly different $(p<0.0001)$. Statistics: weight $\left(p<0.0001, F_{2,10}=79.85\right)$, molt $\left(p=0.6359, F_{2,10}=0.47\right)$, survival $\left(p=0.2538, F_{2,10}=\right.$ 1.58). Means \pm SEM. 


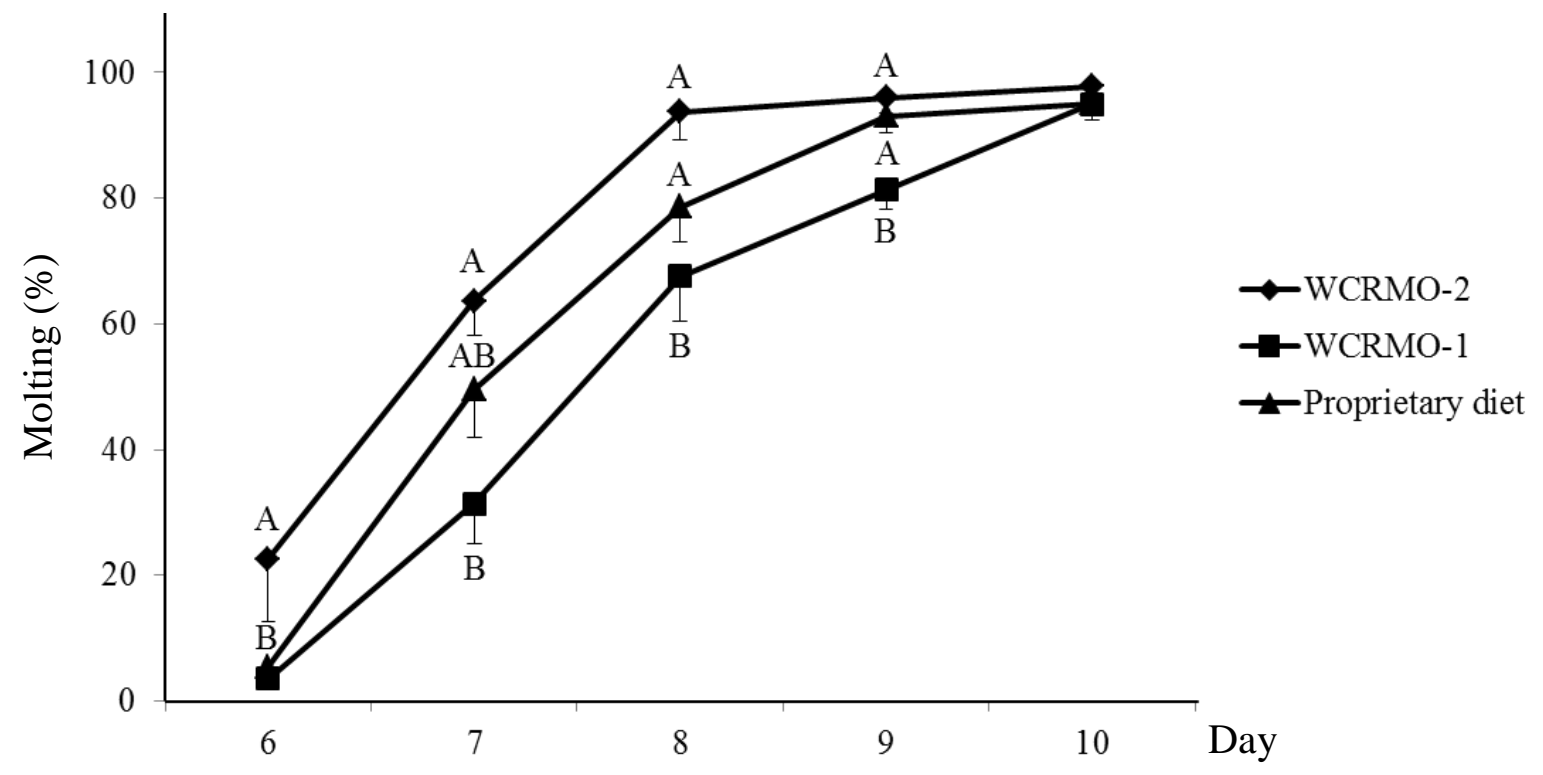

Figure 15. Percent successful completion of molt for western corn rootworm larvae reared on WCRMO-2, WCRMO-1, and a proprietary diet for 10 days. Means with lines followed by different letters are significantly different $(p<0.05)$. Statistics: 6 days $\left(p=0.0076, F_{2,17}=6.59\right), 7$ days $\left(p=0.0081, F_{2,17}=6.47\right), 8$ days $\left(p=0.0146, F_{2,17}=5.48\right), 9$ days $\left(p=0.0336, F_{2,17}=\right.$ $4.17), 10$ days $\left(p=0.6767, F_{2,17}=0.40\right)$. Means \pm SEM. 


\section{References}

Allen, D. M. 1971. Mean square error of prediction as a criterion for selecting variables. Technometrics 13: 469-475.

Anderson, M. J., and P. J. Whitcomb. 2004. RSM simplified: optimizing processes using response surface methods for design of experiments, CRC press.

Anderson, M. J., and P. J. Whitcomb. 2007. Using graphical diagnostics to deal with bad data. Qual. Eng. 19: 111-118.

Ball, H. J., and G. T. Weekman. 1963. Differential resistance of corn rootworms to insecticides in Nehraska and adjoining States. J. Econ. Entomol. 56: 553-555.

Bernklau, E. J., and L. B. Bjostad. 2008. Identification of feeding stimulants in corn roots for western corn rootworm (Coleoptera: Chrysomelidae) larvae. J. Econ. Entomol. 101: 341351.

Cornell, J. A. 2002. Experiments with mixtures: designs, models, and the analysis of mixture data, 3rd ed. John Wiley \& Sons, Inc., New York, USA.

Czitrom, V. 1988. Mixture experiments with process variables: D-optimal orthogonal experimental designs. Commun. Stat. Theory Methods 17: 105-121.

EPA. 2016. EPA docket for corn rootworm resistance management and framework for Bt corn. In U. S. E. P. Agency [ed.]. United States Environmental Protection Agency, Washington, DC, USA.

Eriksson, L., E. Johansson, N. Kettaneh-Wold, C. Wikström, and S. Wold. 2000. Design of experiments. Principles and Applications, Learn ways AB, Stockholm.

Gassmann, A. J., J. L. Petzold-Maxwell, R. S. Keweshan, and M. W. Dunbar. 2011. Fieldevolved resistance to Bt maize by western corn rootworm. PloS one 6: e22629.

Goos, P., B. Jones, and U. Syafitri. 2016. I-Optimal design of mixture experiments. J. Am. Stat. Assoc. 111: 899-911. 
Gray, M. E., T. W. Sappington, N. J. Miller, J. Moeser, and M. O. Bohn. 2009. Adaptation and invasiveness of western corn rootworm: intensifying research on a worsening pest. Ann. Rev. Entomol. 54: 303-321.

Huynh, M. P., L. N. Meihls, B. E. Hibbard, S. L. Lapointe, R. P. Niedz, D. C. Ludwick, and T. A. Coudron. 2017. Diet improvement for western corn rootworm (Coleoptera: Chrysomelidae) larvae. PloS one 12: e0187997.

Kahler, A., A. Olness, G. Sutter, C. Dybing, and O. Devine. 1985. Root damage by western corn rootworm and nutrient content in maize. Agron. J. 77: 769-774.

Kurtz, B., P. Karlovsky, and S. Vidal. 2010. Interaction between western corn rootworm (Coleoptera: Chrysomelidae) larvae and root-infecting Fusarium verticillioides. Environ. Entomol. 39: 1532-1538.

Laake, P. 1975. On the optimal allocation of observations in experiments with mixtures. Scand. J. Stat. 2: 153-157.

Lapointe, S. L., T. J. Evens, and R. P. Niedz. 2008. Insect diets as mixtures: Optimization for a polyphagous weevil. J. Ins. Physiol. 54: 1157-1167.

Lapointe, S. L., R. P. Niedz, and T. J. Evens. 2010a. An artificial diet for Diaprepes abbreviatus (Coleoptera: Curculionidae) optimized for larval survival. Fla. Entomol. 93: 56-62.

Lapointe, S. L., T. J. Evens, R. P. Niedz, and D. G. Hall. 2010b. Artificial diet optimized to produce normative adults of Diaprepes abbreviatus (Coleoptera: Curculionidae). Environ. Entomol. 39: 670-677.

Levine, E., J. L. Spencer, S. A. Isard, D. W. Onstad, and M. E. Gray. 2002. Adaptation of the western corn rootworm to crop rotation: evolution of a new strain in response to a management practice. Am. Entomol. 48: 94-117.

Ludwick, D. C., L. N. Meihls, K. R. Ostlie, B. D. Potter, L. French, and B. E. Hibbard. 2017. Minnesota field population of western corn rootworm (Coleoptera: Chrysomelidae) shows incomplete resistance to Cry34Ab1/Cry35Ab1 and Cry3Bb1. J. Appl. Entomol. 141: $28-40$. 
Ludwick, D. C., L. N. Meihls, M. P. Huynh, A. E. Pereira, B. W. French, T. A. Coudron, and B. E. Hibbard. 2018. A new artificial diet for western corn rootworm larvae is compatible with and detects resistance to all current Bt toxins. Sci. Rep. 8: 5379.

Marrone, P. G., F. D. Ferri, T. R. Mosley, and L. J. Meinke. 1985. Improvements in laboratory rearing of the southern corn rootworm, Diabrotica undecimpuncta howardi Barber (Coleoptera: Chrysomelidae), on an artificial diet and corn. J. Econ. Entomol. 78: 290293.

Meinke, L. J., B. D. Siegfried, R. J. Wright, and L. D. Chandler. 1998. Adult susceptibility of Nebraska western corn rootworm (Coleoptera: Chrysomelidae) populations to selected insecticides. J. Econ. Entomol. 91: 594-600.

Mitchell, P. D. 2011. Costs and benefits of controlling pest Diabrotica in maize in the United States. In, 24th IWGO Conference and 3rd International Conference of Diabrotica Genetics, IWGO, Freiburg, Germany.

Myers, R. H., and D. C. Montgomery. 2002. Response surface methodology: process and product optimization using designed experiments, 2nd ed., Willey, New York, NY, USA.

Myers, R. H., D. C. Montgomery, and C. M. Anderson-Cook. 2016. Response surface methodology: process and product optimization using designed experiments, 4 th ed., John Wiley \& Sons, New York, NY, USA.

Palmer, L., and T. Kommedahl. 1969. Root-infecting Fusarium species in relation to rootworm infestations in corn. Phytopathology 59.

Pereira, A. E., H. Wang, S. N. Zukoff, L. J. Meinke, B. W. French, and B. D. Siegfried. 2015. Evidence of field-evolved resistance to bifenthrin in western corn rootworm (Diabrotica virgifera virgifera LeConte) populations in western Nebraska and Kansas. PloS one 10: e0142299.

Piepel, G. F., and J. A. Cornell. 1987. Designs for mixture-amount experiments. J. Qual. Tech. 19: 11-28.

Pleau, M. J., J. E. Huesing, G. P. Head, and D. J. Feir. 2002. Development of an artificial diet for the western corn rootworm. Entomol. Exp. Appl. 105: 1-11. 
Rose, R. I., and J. M. McCabe. 1973. Laboratory rearing techniques for the southern corn rootworm. J. Econ. Entomol. 66: 398-400.

SAS. 2013. SAS version 9.4, SAS Institute, Cary, N.C, USA.

Scheffé, H. 1958. Experiments with mixtures. Royal Stat. Soc., Series B (Methodological) 20: $344-360$.

Smith, W. F. 2005. Experimental design for formulation. ASA-SIAM series on statistics and applied probability. SIAM, Philadelphia, PA, USA.

Spike, B. P., and J. J. Tollefson. 1988. Western corn rootworm (Coleoptera: Chrysomelidae) larval survival and damage potential to corn subjected to nitrogen and plant density treatments. J. Econ. Entomol. 81: 1450-1455.

Spike, B. P., and J. J. Tollefson. 1989. Relationship of plant phenology to corn yield loss resulting from western corn rootworm (Coleoptera: Chrysomelidae) larval injury, nitrogen deficiency, and high plant density. J. Econ. Entomol. 82: 226-231.

Spike, B. P., and J. J. Tollefson. 1991. Yield response of corn subjected to western corn rootworm (Coleoptera: Chrysomelidae) infestation and lodging. J. Econ. Entomol. 84: $1585-1590$.

Sutter, G. R., J. L. Krysan, and P. L. Guss. 1971. Rearing the southern corn rootworm on artificial diet. J. Econ. Entomol. 64: 65-67.

Weisberg, S. 1985. Applied linear regression, 2nd ed., Wiley \& Sons, New York, NY, USA.

Zukoff, S. N., K. R. Ostlie, B. Potter, L. N. Meihls, A. L. Zukoff, L. French, M. R. Ellersieck, B. Wade French, and B. E. Hibbard. 2016. Multiple assays indicate varying levels of cross resistance in Cry3Bb1-selected field populations of the western corn rootworm to mCry3A, eCry3.1Ab, and Cry34/35Ab1. J. Econ. Entomol. 109: 1387-1398. 


\title{
CHAPTER V: MULTIDIMENSIONAL APPROACH TO FORMULATING A SPECIALIZED DIET FOR NORTHERN CORN ROOTWORM LARVAE
}

\begin{abstract}
The northern corn rootworm (NCR), Diabrotica barberi Smith \& Lawrence, is a major pest of maize (Zea mays L.) in several regions of the United States Corn Belt. Potentially, damage caused by this insect will increase should it develop resistance to management tactics in a manner similar to that observed for the western corn rootworm (WCR). This possibility heightens the importance of monitoring for resistance to maize varieties derived through traditional methods or genetic modification with exogenous toxins. Toxicity bioassays using artificial diet have proven to be valuable for monitoring resistance in WCR to toxins produced by Bacillus thuringiensis (Bt). No artificial diet has been developed specifically for NCR larvae. The best artificial diet for NCR to date has been a diet developed for the WCR. We developed a specialized diet for NCR using an iterative approach. Screening designs including 8 diet components were performed to identify the principal nutritional components contributing to multiple developmental parameters (survival, weight, and molting). We then applied mixture designs coupled with response surface modeling to optimize a blend of those components. Finally, we validated an improved NCR diet formulation that supports approximately $97 \%$ survival and molting, and a 150\% increase in larval weight after 10 days of feeding compared with the best previously published artificial diet. This formulation appears suitable for use in diet bioassays as a tool for evaluating the resistance of NCR populations to insecticides.
\end{abstract}




\section{Introduction}

The northern corn rootworm (NCR), Diabrotica barberi Smith \& Lawrence, is a major insect pest of maize (Zea mays L.) in the North American Corn Belt. Combined with the western corn rootworm (WCR), Diabrotica virgifera virgifera LeConte, these corn rootworms are responsible for more than $\$ 1$ billion annually due to yield reduction and control costs in the United States (Mitchell 2011). Rootworm larval feeding on maize roots can cause disruption to nutrient and water flow (Kahler et al. 1985) and facilitation of pathogen susceptibility (Palmer and Kommedahl 1969, Kurtz et al. 2010) and grain losses due to plant lodging (Spike and Tollefson 1988, 1989, 1991) that result in yield reduction (Spike and Tollefson 1989, 1991). This insect is highly adaptive and has developed resistance to several management tactics, including soil insecticides (Hamilton 1965) and crop rotation (Krysan et al. 1986). The latest management tools, transgenic maize hybrids expressing insecticidal proteins from Bacillus thuringiensis (Bt) Berliner, were recently documented to falter due to resistance of WCR (Gassmann et al. 2011, Zukoff et al. 2016, Ludwick et al. 2017) to the toxins. Since the targeted pests of $B t$ corn are Diabrotica species including both WCR and NCR, the recent increase in NCR populations in some areas causes concern that a similar adaptation may occur with NCR to Bt corn and/or other management practices.

The United States Environmental Protection Agency (EPA) requires monitoring programs to detect the development of resistance in insect populations. Diet toxicity bioassays, whereby insects are exposed to toxins on artificial diet, can be a valuable component of such programs. However, this requires an artificial diet capable of supporting insect growth and development. Unfortunately, artificial diets for use in evaluating resistance to Bt corn in wildtype populations of NCR are lacking. Because no artificial diet has been developed specifically 
for NCR, an artificial diet for southern corn rootworm (SCR), Diabrotica undecimpunctata howardi Barber, has been used in diet toxicity bioassays (Oyediran et al. 2016). Development of an NCR diet to support development (e.g., survival and molting) would be helpful in detecting resistance development of NCR populations.

Diet development for Diabrotica species started with a formulation for larvae of SCR, which is a generalist that feeds on over 100 different plants. The first SCR formulation developed by Sutter et al. (1971) consisted of wheat germ and casein as key ingredients. This formulation supported SCR development from egg to adult, but development was slower and fecundity was lower for larvae reared on the diet compared with larvae reared on corn roots (Sutter et al. 1971). SCR diet improvements were reported by Rose and McCabe (1973) and Marrone et al. (1985) by altering linseed oil, sucrose, antibiotics, and potassium hydroxide. That formulation improved SCR development and approached that of larvae reared on corn roots after 6 generations of selection on the diet. The SCR diet has been used in WCR diet bioassays, although growth rates for WCR were poorer than that of SCR and microbial contamination was a major issue (Pereira et al. 2016). Later, several modifications of this diet were made by industry and a high level of antibiotics was added into the SCR diet to avoid contamination (Zhao et al. 2016).

An initial formulation for WCR larvae was made by Pleau et al. (2002) with modifications made to a diet for SCR (Marrone et al. 1985) by adjusting amounts of several ingredients (i.e., wheat germ, linseed oil, and potassium hydroxide), removing formalin and adding corn root powder. The Pleau et al. diet doubled the weight of WCR larvae compared with larvae reared on the SCR formulation (Pleau et al. 2002). Later, the Pleau et al. diet was improved by Huynh et al. (2017), which resulted in a formulation that further increased the 
weight of WCR larvae and increased both survival and molting after 11 days compared with the first WCR formulation. The Pleau et al. diet was improved by altering several components (i.e., sucrose, wheat germ, casein, cellulose, corn root powder, linseed oil, and agar) and adding wheat germ oil. The Huynh et al. diet had nearly zero contamination after 11 days through clean laboratory practices (Huynh et al. 2017). This diet was also compatible with all current $\mathrm{Bt}$ proteins targeting WCR larvae (Ludwick et al. 2018).

Past approaches to formulate and develop insect diets involved one-factor-at-a-time (OFAT) and multivariate experiment designs based on mixture designs. For OFAT designs, each diet component was varied independently. Because changing the amount of one factor is confounded with changes in the proportions of ingredients in a mixture, the OFAT design is not capable of identifying an optimal blend of diet components (Niedz and Evens 2016). The multivariate geometric approach in combination with a mixture design allows simultaneous varying of multiple diet components and reveals important interactive effects of diet components on several measured responses at a same time (Lapointe et al. 2008, Raubenheimer 2011, Huynh et al. 2017). Henri Scheffé (1958) developed mathematical models specifically for response surface modeling based on mixture designs and the corresponding computations are simplified by modern software for design of experiments (Lapointe et al. 2008, Anderson and Whitcomb 2004). The application of response surface methodology combined with a mixture design has been of great value for diet improvement and optimization for WCR (Huynh et al. 2017) and another coleopteran insect (Lapointe et al. 2008, Lapointe et al. 2010b).

For the current paper, we first compared the performance of 5 published artificial diets for rearing NCR and identified key diet ingredients in the best diet in a 8-component experiment. We then screened 8 protein sources to identify best options. Finally, we optimized 
specific blends to obtain our desired responses. We combined the response surface method with a mixture design to identify the main drivers of larval development by maximizing larval performance (larval survival, development, and weight) while limiting diet contamination compared with the performance of NCR on existing diets developed for related species (WCR and SCR).

\section{Materials and methods}

\section{Insects}

NCR eggs (a diapausing strain) were obtained from the North Central Agricultural Research Laboratory in Brookings, South Dakota. NCR eggs in 70 mesh sieved-soil were incubated at a constant $25^{\circ} \mathrm{C}$ in complete darkness and then washed out of the soils though a 60 mesh sieve (Hogentogler \& Co. Inc., Columbia, MD) with water after the first eggs hatched. The remaining eggs were surface-treated using a procedure described by Pleau et al. (2002). Washed eggs were first immersed in undiluted lysol ${ }^{\circledR}$ (Reckitt Benckiser, LLC, Parsippany, NJ) for 3 minutes and then triple rinsed with distilled water. Next, the eggs were immersed in $10 \%$ formalin (HT501128, Sigma Aldrich, St. Louis, MO) for 3 minutes and then triple rinsed with distilled water. Finally, the eggs were dispensed onto a coffee filter paper (Pure Brew, Rockline Industries, Sheboygan, WI) that was placed inside a 16 oz. cup (LG8RB-0090, Solo Cup Company, Lake Forest, IL) with a lid (DM16R-0090, Solo Cup Company) containing several holes made by a number zero insect pin. The eggs were then incubated at $25^{\circ} \mathrm{C}$ in darkness. Larvae that hatched within 24 hours were used for the insect bioassays. 


\section{Diet preparation}

Frontier SCR diets (F9800B \& F9757B, Frontier, Newark, DE) were purchased and poured according to procedures by manufacturers. Other diets including the Marrone et al. diet, the Pleau et al. diet and the WCRMO-1 diet were made using a procedure described in Pleau et al. (2002), with some modifications by Huynh et al. (2017). The solution of distilled water and agar (A7002, Sigma-Aldrich) in a $400 \mathrm{ml}$ glass beaker was boiled in a microwave for 2 minutes and then poured into a blender (Hamilton Beach, Inc., Model 51101BZ) and placed in a biological safety cabinet (Nuaire, Biological safety cabinet). When the molten agar had cooled to $65^{\circ} \mathrm{C}$, dry diet ingredients, i.e., corn gluten meal (49760, Sigma-Aldrich), cottonseed meal (Down-To-Earth, Eugene, OR), plant protein (Perfect supplements, Coventry, RI), whey protein (ON, Downers Grove, IL), perfect amino (Bodyhealth, Clearwater, FL), yeast extract (BP1422, Fisher Scientific, Fair Lawn, NJ), egg powder (Judee's gluten free, Columbus, OH), casein (1100, Bio-Serv), wheat germ (1661, Bio-Serv, Flemington, NJ), cellulose (3425, Bio-Serv), glucose (D16, Fisher Scientific), sucrose (04821721, MP Biomedicals, Santa Ana, CA), corn root powder (USDA-ARS, Columbia, MO), salt mix (F8680, Bio-Serv), vitamin mix (V1007, Sigma-Aldrich), methyl paraben (H5501, Sigma-Aldrich), cholesterol (C8503, Sigma-Aldrich), and sorbic acid (S1626, Sigma-Aldrich)), were added (if applicable) to the blender and mixed thoroughly for 30 seconds. Next, liquid ingredients, i.e., linseed oil (430021, Sigma-Aldrich), wheat germ oil (W1000, Sigma-Aldrich), streptomycin (612240500, Across, Morris Plains, New

Jersey), chlortetracycline (C4881, Sigma-Aldrich), green food coloring (Butler, Lancaster, PA) if applicable, were added to the mixture and blended for 30 seconds. The $\mathrm{pH}$ of the diet was monitored with indicator strips (Whatman ${ }^{\circledR}$ 09-876-18, GE Healthcare Bio-Sciences, Pittsburg, PA) and adjusted a $\mathrm{pH}$ of 9.0 by the addition of $10 \% \mathrm{KOH}(\mathrm{w} / \mathrm{v})(\mathrm{P} 250$, Fisher Scientific). The 
mixture was poured into a $750 \mathrm{ml}$ glass beaker and slowly stirred at $65^{\circ} \mathrm{C}$ on a stirring hot plate (Thermo scientific, Cimarec ${ }^{\mathrm{TM}}$ ). Using a repeater pipette (Eppendorf repeater plus), the diet mixture was pipetted into each well (200 $\mu 1 /$ well) of a 96-well plate (3370, Corning Inc., Corning, NY) and then excess moisture was allowed to evaporate for $30 \mathrm{~min}$. Diet plates were stored at $4^{\circ} \mathrm{C}$ and used within a week.

\section{Insect diet bioassays}

The diet bioassays were conducted as described in Huynh et al. (2017). All materials used in the diet assays were surface-treated via exposure to UV light for $10 \mathrm{~min}$ in a biological cabinet. Each formulation was tested in a 12-well row of a 96 well plate and values from the 12 sub-samples were averaged to yield a single replicate. Each formulation was replicated at least 4 times. Eight different formulations were randomly assigned on the plate. One larva $(<24 \mathrm{~h}$ after hatching) was placed in each well of the diet plate using a fine paintbrush. A sealing film (TSSRTQ-100, Excel Scientific, Inc., Victorville, CA) was used to cover the plate and one vent hole was made per well using an insect pin (size 0). The plates were stored at $25^{\circ} \mathrm{C}$ in darkness for 10 days. Collections made at 10 days were used to determine differences in survival, weight and

molting to the $2^{\text {nd }}$ instar. Larval survival, time of molt to $2^{\text {nd }}$ instar, and diet contamination were recorded. Live larvae were collected and pooled within a replication into $95 \%$ ethanol, dried in an oven (602752, Blue M Therm Dry Bacteriological Incubator) at $50^{\circ} \mathrm{C}$ for 2 days, and then were weighed using a micro balance (MSU6.6S-000-DM, Sartorius Lab Instruments GmbH \& Co. KG, Goettingen, Germany). 


\section{Experimental approach}

An iterative approach was used to develop an artificial diet for NCR. An initial experiment was conducted by comparing five current rootworm diets. A screening design was conducted to explore the nutritional profile of NCR by varying 8 components using a mixture design. Polynomial equations were generated to describe the impact of diet ingredients on the measured responses (i.e., larval weight, molting, survival, and contamination). A screening experiment was used to characterize the effects of 8 different protein sources on the measured responses using an 8-component mixture design. A mixture-amount design (Cornell 2002) was also used to identify an optimum formulation of 3 protein ingredients that had the greatest effect on the measured responses. The predicted optimal blend was then validated in a comparison experiment.

\section{Comparison of five rootworm diets}

Three SCR diets included the two Frontier diets (F9800B \& F9757B, Frontier, Newark, DE) plus the Marrone et al. diet (Marrone et al. 1985). The two WCR diets included Pleau et al. diet (Pleau et al. 2002) and WCRMO-1 (Huynh et al. 2017). An improved formulation was chosen based on overall larval performance (i.e., larval weight, molting, and survival) after $10 \mathrm{~d}$ of feeding in diet assays.

\section{8-component mixture design for basic nutrition profile}

Since the WCRMO-1 diet was the best of the five public rootworm diets evaluated, improvements started from this diet. It consisted of 18 diet ingredients (Huynh et al. 2017). An exploratory experiment to identify the basic nutritional profile of NCR simultaneously varied 8 
diet components including casein, cellulose, corn root powder, wheat germ, wheat germ oil, linseed oil, and agar. Other ingredients (vitamin and salt blends, preservatives and antibiotics) were kept constant at the levels in the WCRMO-1 (Huynh et al.). No effort was made to deconvolute the mixtures of Vanderzant vitamin and Wesson salt that are widely used in insect diets. The exploratory design was created with Design-Expert (v.10.0, Stat-Ease, Inc., Minneapolis, MN) resulting in 24 design points including vertex, center, 7-blend, and axial check blend points (Table 15, (Anderson and Whitcomb 2004)). The design included 7 model, 11 lack of fit, and 5 pure error degrees of freedom (Myers and Montgomery 2002).

\section{8-protein screening design}

The 8-component mixture experiment indicated casein, corn root powder, lipid ingredients and agar had positive effects on both NCR larval weight and molting. Survival was removed as a criterion due to more than $90 \%$ survival across almost all design points. Lipid ingredients (wheat germ oil and linseed oil) were removed due to their minor contributions in WCR diets (Huynh et al. 2017). Glucose was substituted for sucrose because glucose is a main component of sugar in maize roots (Bernklau and Bjostad 2008). In this study, 8 different protein sources were concurrently screened to characterize their contributions to larval performance (weight, molting, and survival) by constructing an I-optimal mixture design (Laake 1975, Goos et al. 2016). This design consisted of 32 design points with 7 model, 17 lack of fit and 5 pure error degrees of freedom (Table 16). Concentrations of other ingredients were the same as for WCRMO-1 (Table 17). 


\section{3-protein mixture design}

The 3-proteins responsible for the largest effects on NCR larval weight, molting, and survival were used to construct a D-optimal mixture-amount design (Czitrom 1988, Cornell 2002) sufficient for a Scheffé quadratic-quadratic polynomial response surface model. This design consisted of 32 design points with 17 model, 6 lack of fit and 8 pure error degrees of freedom (Table 18). Other ingredients were kept constant as shown in Table 17. Optimal blends were predicted using overlay response surface plots combined with a simplex hill-climbing algorithm included in Design-Expert ${ }^{\mathrm{TM}}$ software (Lapointe et al. 2008).

\section{Diet optimization}

A formulation for maximizing larval performance (survival, molting, and weight) was calculated with Design-Expert $^{\mathrm{TM}}$ (Stat-Ease, Inc., Minneapolis, MN). This software used overlay response surface and direct search methods (Derringer 1980) to maximize the desirability function (Myers et al. 2016).

\section{Model validation}

All three measures of larval performance (survival, molting, and weight) identified by the response surface mixture model were compared with those of larvae reared on WCRMO-1 $\operatorname{diet}$ (Huynh et al. 2017).

\section{Statistical analyses}

Survival and molting data were generated by dividing the number of live larvae and successful larval molts from $1^{\text {st }}$ to $2^{\text {nd }}$ instar per replicate, respectively, by the initial number of 
larvae infested and multiplying by 100 to obtain percentages. Weight data were generated by dividing total dry weight per replicate by the number of live larvae while dead larvae were recorded as 0 in weight.

In the diet comparison experiments, measured parameters of larval performance on the rootworm diets at 10 days after infestation were analyzed as a randomized complete block design using PROC MIXED in SAS (SAS 2013). All percent variables were arcsine square-root transformed prior to the analysis to meet assumptions of normality and homoscedasticity

In the mixture experiments, all possible models from $1^{\text {st }}$ degree to $4^{\text {th }}$ degree polynomials for each measured response of larval performance (larval survival, proportion of successful larval molts and larval weight) were generated with Design Expert ${ }^{\circledR}$. Model selection as described in Lapointe et al. (2008). Briefly, the criteria included a lack of aliased terms, low residual values, low model $P$-value, nonsignificant lack of fit, low standard deviation, high $\mathrm{R}^{2}$, $\mathrm{R}^{2}$ adj and $\mathrm{R}_{\text {pred }}^{2}$ (Lapointe et al. 2008), close agreement between $\mathrm{R}_{\text {adj }}^{2}$ and $\mathrm{R}_{\text {pred }}^{2}$, and a low PRESS value (Allen 1971, Myers and Montgomery 2002). When two or more models were satisfactory, the selected model was then further evaluated according to adequacy tests as described by Anderson and Whitcomb (Anderson and Whitcomb 2004, Anderson and Whitcomb 2007).

\section{Results}

\section{Comparison of five rootworm diets}

Larval survivorship to 10 days was significantly different $\left(P<0.0001, F_{4,24}=247.4\right)$ among the five tested diets (Fig. 16A). There was no significant difference between WCRMO-1 and Frontier 9800B. The Pleau et al. diet had significantly lower survivorship than WCRMO-1 
and Frontier 9800B whereas no larvae survived to 10 days when reared on Frontier 9757B or the Marrone et al. diet. Average larval dry weight after 10 days varied significantly across diets $\left(p<0.0001, F_{4,24}=122.6\right)$. The WCRMO-1 diet had significantly higher larval dry weight than other diets while the Frontier F9800B diet had significantly lower larval dry weight than the Pleau et al. diet (Fig. 16B). Significantly more NCR larvae molted from $1^{\text {st }}$ instar to $2^{\text {nd }}$ instar when reared on WCRMO-1 than on any other diet $\left(P<0.0001, F_{4,24}=189.5\right)($ Fig. 16C). The Pleau et al. diet had more larvae molt than Frontier F9800B. Overall, WCRMO-1 supported better NCR larval performance than other diets.

\section{8-component screening experiment for basic nutrition profile}

The 8-component mixture design produced significant response surface models for all three measurement criteria; larval weight $\left(P<0.0001, F_{9}=37.9\right)$, molting $\left(P<0.0001, F_{8}=\right.$ 47.3), and survival $\left(P<0.0001, F_{8}=19.1\right)$. The 8 diet components that were varied at a same time included: agar, sucrose, wheat germ, casein, cellulose, corn root powder, wheat germ oil and linseed oil. Models for weight and survival had insignificant lack of fit whereas there was a significant lack of fit for molting due to a very small value of pure error. All models were improved by stepwise regression and had $\mathrm{R}^{2}, \mathrm{R}_{\text {adj }}^{2}$ and $\mathrm{R}_{\text {pred }}^{2}$ clustered with the differences $<0.2$ (Table 19), indicating good predicted models. The relationships between diet ingredients and larval performance (Fig. 17), depict the effect of increasing the proportion of one component in relation to a reference blend while the relative proportions of all of the other diet components are kept constant (Lindig and Malone 1973, Smith 2005) and are called Cox plots. The Cox plot was generated by inverted high and low values, which showed that the slope of the line indicated the inverted direction and magnitude of the influence of the individual factors on the measured 
response variable, e.g., larval weight. Models for weight and molting indicated that casein, corn root powder and two lipid components had the greatest effects on improving these criteria (Figs. 17A-B) whereas a model for survival revealed that wheat germ, corn root powder and wheat germ oil were the the primary drivers (Fig. 17C). Increases in the two lipid components had minor contributions to improving WCR larval performance (Huynh et al. 2017). Since almost all diet blends had $>90 \%$ survival, we removed survival as a desired trait for improvement. Casein was the main protein source of the diet formulation, and was an important driver for weight and molting, so next we tried alternative protein sources.

\section{8-protein mixture experiment}

The protein screening design yielded significant response surface models for larval weight $\left(P<0.0001, F_{12}=35.6\right)$, molting $\left(P<0.0001, F_{9}=16.4\right)$, and survival $\left(P<0.0001, F_{8}=\right.$ 33.7) by varying 8 different protein sources: corn gluten meal, cottonseed meal, casein, plant protein, whey protein, perfect amino, yeast extract, and egg powder (Table 20). Models for weight had insignificant lack of fit whereas models for molting and survival had significant lack of fit due to very small values of pure error. $\mathrm{R}^{2}, \mathrm{R}_{\text {adj }}^{2}$ and $\mathrm{R}_{\text {pred }}^{2}$ of all models were clustered with the differences $<0.3$. The relationships between protein sources and larval performance (Fig. 18) indicate animal protein sources (casein, egg powder, and whey protein) had the greatest effects on improving all three criteria of larval performance (Figs. 18A-C). In addition, plant protein had positive effects on larval performance and cottonseed meal had positive effect on survival. In contrast, corn gluten meal, perfect amino, and yeast extract had negative effects on all developmental traits. In Figure 18, the slope shows the direction and magnitude of the impact of each ingredient on all measured responses. 


\section{3-protein mixture-amount experiment}

The 3-protein mixture-amount was used to identify an optimal 3-component blend for larval weight $\left(P<0.0001, F_{5}=10.2\right)$, molting $\left(P<0.0001, F_{8}=14.1\right)$, and survival $(P<0.0001$, $\left.F_{6}=8.8\right)$ using: casein, egg powder, and whey protein (Table 21$) . \mathrm{R}^{2}, \mathrm{R}^{2}$ adj and $\mathrm{R}^{2}$ pred of all models were clustered with the differences $<0.3$. Lack of fit of models for weight was significant due to very small values of pure error while models for molting and survival had insignificant lack of fit. The relationships between protein sources and larval performance were shown in ternary plots (Fig. 19). Possible combinations of proportions of the mixtures of three components were revealed in the ternary plots. The magnitude of the response variables is coded in color and can be envisioned as perpendicular to the page as indicated by labelled isobars (Figs. 19A-C).

Models for weight and molting indicated that a blend of casein and egg powder was optimal for maximum weight and molting (Figs. 19A \& B). High proportions of casein resulted in a decrease in all developmental traits. Whey protein had positive effect on survival (Fig. 19C), but a negative effect on weight and molting when added as a component of the protein mixture. Results also showed very strong blending effects of egg powder and casein on larval weight and molting, but an antagonistic blending effect of these components on survival (Fig. 20). Such a trade-off between survival and weight gain has been shown to occur in other beetles reared on artificial diets (Lapointe et al. 2010a).

The contour plots (Figs. 19D-F) represent the interactive effects of casein and egg powder and the amount of mixture while proportions of whey protein to diet mixture were constant at 0.34 to 1 . The labelled isobars and color indicate the magnitude of the measured response. All contour plots indicated that a higher total amount of the mixture could yield a 
better formulation. Ratios of egg powder:casein at 4:1, 1:1, 3:1 could produce the best formulation for weight, molting and survival, respectively (Figs. 19D-F).

\section{Contamination}

Since all experiments had minor contamination $(<1 \%)$, there was no evidence for a relationship between contamination and diet ingredients. Similarly low contamination rates were seen in Huynh et al. (2017) and Ludwick et al. (2018) .

\section{Diet optimization}

The diet development for NCR larvae produced a formulation, referred to hereafter as NCRMO-1 (Table 22). This formulation was calculated with Design-Expert ${ }^{\mathrm{TM}}$ by optimizing key ingredients based on the integrated evaluation of all developmental traits for WCR. An overlay graph comprised of the contour plots from each response laid on top of each other was generated to identify a region where all measured responses were simultaneously maximized (Fig. 19G).

\section{Model validation and diet improvement}

The NCRMO-1 diet yielded better larval performance compared with the best previously published diet for WCR, WCRMO-1. The formulation for NCR larvae was modified from WCRMO-1, with several modifications including the elimination of corn root powder, linseed oil and wheat germ oil, substitution of sucrose with glucose, and addition of egg powder (Table 22). Other ingredients were at the same level as in WCRMO-1 (Huynh et al. 2017). After 10 days of feeding on NCRMO-1, larval dry weight, survival and molting rate were $0.44 \mathrm{mg}$, 
97\%, and $97 \%$ compared to $0.28 \mathrm{mg}, 98 \%$ and $96 \%$, respectively when reared on WCRMO-1

(Fig. 21).

\section{Discussion}

We applied a multivariate geometric approach in combination with a n-dimensional mixture design to develop the first artificial diet formulated specifically for rearing NCR. The algorithm used consisted of five steps including evaluation of existing diets, identification of basic nutritional profiles, exploration of key nutrients, maximizing key ingredients, and evaluation of formulations, as presented in Figure 22. The application of geometric and mathematical approaches including response surface modeling (Lee et al. 2008), response surface modeling combined with n-dimensional mixture design (Lapointe et al. 2008, Lapointe et al. 2010b, Huynh et al. 2017), and orthogonal experimental designs (Tan et al. 2013) have been used for improvement of existing diets for other insect species. By applying the response surface methodology based on a multivariate mixture design, we identified key ingredients and then optimized the key ingredients to create the best formulation that maximized life history criteria (survival, molting, and weight) for NCR.

Our results indicated that protein ingredients were key components that had the greatest effect on larval performance i.e., changing the concentration of these ingredients strongly affected the life history parameters. In contrast, other ingredients (e.g., wheat germ, cellulose, sucrose, linseed oil, wheat germ oil, and agar) had little or negative contributions on the overall response parameters (Fig. 17). We found that NCR larvae performed better with animal proteins compared with 8 other protein sources tested, including plant and yeast proteins. The addition of animal protein sources (i.e., casein, egg powder, whey protein) and plant protein 
improved all measured responses (weight, molting, and survival) whereas the inclusion of other protein ingredients (i.e., corn gluten meal, cottonseed meal, perfect amino, and yeast extract) resulted in a decrease in all three measured responses, except for a positive effect of cottonseed meal on survival (Figs. 18A-C). The nonlinear blending effects indicated casein and egg powder were the most important optimize protein sources among the three animal protein sources tested (Figs. 19D-F). Casein at high proportions (Fig. 18C) caused a decrease in survival when changed in combination with egg powder and whey protein. Our results revealed that wheat germ had a complex relationship with the life history parameters. Wheat germ at high proportions improved survival, but caused a decrease in molting (Figs. 17B-C). Similar effects of wheat germ on insect performance have been reported previously for WCR larvae (Pleau et al. 2002, Huynh et al. 2017). Wheat germ is documented as a key ingredient for nutritional value, digestibility, bioavailability, and palatability, but at high proportions can cause detrimental effects on insect development (Cohen 2015).

We found that corn root powder was a key ingredient that had a strong positive influence on life history parameters. The addition of corn root powder increased all larval performance measures such as weight, molting and survival (Figs. 17A-C). The important role of corn root powder was also documented in WCR diets, wherean increase in proportion of root powder improved molting and survival, but caused a decrease in weight when added at high concentrations (Pleau et al. 2002, Huynh et al. 2017). Corn roots contain host recognition cues and feeding stimulant, but also have a repellent compound of WCR larvae (Bernklau and Bjostad 2008, Bernklau et al. 2015, Bernklau et al. 2016). Corn root powder is not currently available for purchase, making it a limiting factor for users. In this study, we identified and characterized and 
optimize the key protein sources (Figs. $18 \& 19$ ) that enabled to eliminate corn root powder, making our formulation more widely applicable.

Our results revealed significant differences in NCR larval development among rootworm diets (Figs. 16 \& 21). WCRMO-1, a superior diet for WCR (Huynh et al. 2017), supported NCR larval performance better than SCR diets and another WCR diet. This is possibly because both WCR and NCR are nearly monophagous on corn roots, although they can also survive on some grass species (Oyediran et al. 2004, Oyediran et al. 2008). There was zero NCR survival to day 10 when feeding on diets containing approximately $1 \%$ formalin, e.g., Frontier F9757B and Marrone et al. diets. A detrimental effect of formalin on weight was reported previously for WCR. Larval weight reduced to half when feeding on diets with an addition of $1 \%$ formalin (Pleau et al. 2002). In contrast, at 10 days post infestation, survival and molting rate of NCR larvae reared on NCRMO-1 (without formalin) were approximately $97 \%$ and larval dry weight increased $150 \%$ fold with NCRMO-1 as compared to WCRMO-1 (Fig. 21).

Although the ultimate goal of insect artificial diet research might be to serve as a comparable substitute for natural foods (Lapointe et al. 2008), only a few diet formulations can be considered to have achieved that goal over the past 60 years (Cohen 2015). However, artificial diets are also desired to enable the study of insect species of interest (Beck and Stauffer 1950, Vanderzant and Reiser 1956a, b), to reduce labor or space (Beck and Stauffer 1950), to reduce time for host plant rearing (Vanderzant et al. 1956), and to provide a standardized diet for insect bioassays (Huynh et al. 2017). This is the first report of a diet formulation developed specifically for NCR larvae. NCRMO-1 supported high survival, weight, and percentage of larvae molted to second instar after 10 days. Additionally, this formulation had basically zero 
contamination. Consequently, this formulation is suitable for use in diet bioassays for monitoring resistance programs of NCR, and possibly for the study of basic NCR biology and physiology. 
Table 15. Diet blends of 8 components varied in the mixture exploratory design to rear northern corn rootworm larvae.

\begin{tabular}{crrrrrrrr}
\hline Diet blend \# & $\begin{array}{r}\text { Agar } \\
(\mathrm{g})\end{array}$ & $\begin{array}{r}\text { Sucrose } \\
(\mathrm{g})\end{array}$ & $\begin{array}{r}\text { Wheat germ } \\
(\mathrm{g})\end{array}$ & $\begin{array}{r}\text { Casein } \\
(\mathrm{g})\end{array}$ & $\begin{array}{r}\text { Cellulose } \\
(\mathrm{g})\end{array}$ & $\begin{array}{r}\text { Corn root } \\
(\mathrm{g})\end{array}$ & $\begin{array}{r}\text { Wheat germ oil } \\
(\mathrm{ml})\end{array}$ & $\begin{array}{r}\text { Linseed oil } \\
(\mathrm{ml})\end{array}$ \\
\hline 1 & 1 & 5 & 8 & 0 & 1.85 & 0 & 0.1 & 0.1 \\
2 & 1 & 5 & 7.95 & 0 & 0 & 2 & 0 & 0.1 \\
3 & 2 & 0 & 6.95 & 5 & 0 & 2 & 0 & 0.1 \\
4 & 2 & 0 & 1.85 & 5 & 5 & 2 & 0.1 & 0.1 \\
5 & 2 & 5 & 1.95 & 5 & 0 & 2 & 0.1 & 0 \\
6 & 1 & 5 & 0 & 5 & 4.95 & 0 & 0 & 0.1 \\
7 & 2 & 5 & 1.95 & 0 & 5 & 2 & 0 & 0.1 \\
8 & 2 & 0.95 & 8 & 0 & 5 & 0 & 0.1 & 0 \\
9 & 1 & 5 & 8 & 0.05 & 0 & 2 & 0 & 0 \\
10 & 1.48 & 2.74 & 5.18 & 2.74 & 2.74 & 1.07 & 0.05 & 0.05 \\
11 & 1.48 & 2.74 & 5.18 & 2.74 & 2.74 & 1.07 & 0.05 & 0.05 \\
12 & 1 & 0 & 7.95 & 0 & 5 & 2 & 0.1 & 0 \\
13 & 2 & 5 & 2.05 & 0 & 5 & 2 & 0 & 0 \\
14 & 2 & 0 & 8 & 5 & 1.05 & 0 & 0 & 0 \\
15 & 1 & 0 & 2.85 & 5 & 5 & 2 & 0.1 & 0.1 \\
16 & 1.48 & 2.74 & 5.18 & 2.74 & 2.74 & 1.07 & 0.05 & 0.05 \\
17 & 2 & 5 & 3.95 & 5 & 0 & 0 & 0.1 & 0 \\
18 & 1 & 0 & 7.95 & 5 & 0 & 2 & 0.1 & 0 \\
19 & 1 & 5 & 0.05 & 5 & 5 & 0 & 0 & 0 \\
20 & 2 & 0 & 8 & 0.95 & 5 & 0 & 0 & 0.1 \\
21 & 2 & 5 & 8 & 0.85 & 0 & 0 & 0.1 & 0.1 \\
22 & 1.48 & 2.74 & 5.18 & 2.74 & 2.74 & 1.07 & 0.05 & 0.05 \\
23 & 2 & 5 & 1.95 & 5 & 0 & 2 & 0.1 & 0 \\
24 & 1 & 5 & 0.05 & 5 & 5 & 0 & 0 & 0 \\
\hline
\end{tabular}


Table 16. Diet blends of 8-proteins (in grams) varied in the mixture screening design to rear northern corn rootworm larvae.

\begin{tabular}{crrrrrrrr}
\hline Diet blend \# & $\begin{array}{r}\text { Corn gluten } \\
\text { meal }\end{array}$ & $\begin{array}{r}\text { Cottonseed } \\
\text { meal }\end{array}$ & Casein & $\begin{array}{r}\text { Egg } \\
\text { powder }\end{array}$ & $\begin{array}{r}\text { Plant } \\
\text { protein }\end{array}$ & $\begin{array}{r}\text { Perfect } \\
\text { Amino }\end{array}$ & $\begin{array}{r}\text { Yeast } \\
\text { extract }\end{array}$ & $\begin{array}{r}\text { Whey } \\
\text { protein }\end{array}$ \\
\hline 1 & 0.38 & 0.38 & 0.38 & 0.38 & 0.37 & 0.37 & 0.37 & 0.37 \\
2 & 0.43 & 0 & 0.43 & 0.43 & 0.43 & 0.43 & 0.43 & 0.42 \\
3 & 0 & 0.43 & 0.43 & 0.43 & 0.43 & 0.43 & 0.43 & 0.42 \\
4 & 0.43 & 0.43 & 0.43 & 0.43 & 0 & 0.43 & 0.43 & 0.42 \\
5 & 0.43 & 0.43 & 0.43 & 0 & 0.43 & 0.43 & 0.43 & 0.42 \\
6 & 0.38 & 0.38 & 0.38 & 0.38 & 0.37 & 0.37 & 0.37 & 0.37 \\
7 & 0 & 0 & 0 & 0 & 0 & 0 & 3 & 0 \\
8 & 0.43 & 0.43 & 0.43 & 0.43 & 0.43 & 0 & 0.43 & 0.42 \\
9 & 0.38 & 0.38 & 0.38 & 0.38 & 0.37 & 0.37 & 0.37 & 0.37 \\
10 & 3 & 0 & 0 & 0 & 0 & 0 & 0 & 0 \\
11 & 0.38 & 0.38 & 0.38 & 0.38 & 0.37 & 0.37 & 0.37 & 0.37 \\
12 & 0.19 & 0.19 & 0.19 & 0.19 & 0.19 & 1.67 & 0.19 & 0.19 \\
13 & 0 & 0 & 0 & 3 & 0 & 0 & 0 & 0 \\
14 & 0.19 & 0.19 & 0.19 & 1.67 & 0.19 & 0.19 & 0.19 & 0.19 \\
15 & 0.43 & 0.43 & 0 & 0.43 & 0.43 & 0.43 & 0.43 & 0.42 \\
16 & 1.67 & 0.19 & 0.19 & 0.19 & 0.19 & 0.19 & 0.19 & 0.19 \\
17 & 0 & 0 & 3 & 0 & 0 & 0 & 0 & 0 \\
18 & 0.19 & 0.19 & 1.67 & 0.19 & 0.19 & 0.19 & 0.19 & 0.19 \\
19 & 0 & 0 & 0 & 0 & 0 & 0 & 0 & 3 \\
20 & 0 & 0 & 0 & 0 & 0 & 3 & 0 & 0 \\
21 & 0.19 & 1.67 & 0.19 & 0.19 & 0.19 & 0.19 & 0.19 & 0.19 \\
22 & 0.19 & 0.19 & 0.19 & 0.19 & 0.19 & 0.19 & 0.19 & 1.67 \\
23 & 0.43 & 0.43 & 0.43 & 0.43 & 0.43 & 0.43 & 0 & 0.42 \\
24 & 0 & 0 & 0 & 0 & 3 & 0 & 0 & 0 \\
25 & 0.19 & 0.19 & 0.19 & 0.19 & 1.67 & 0.19 & 0.19 & 0.19 \\
26 & 0.19 & 0.19 & 0.19 & 0.19 & 0.19 & 0.19 & 1.67 & 0.19 \\
27 & 0.38 & 0.38 & 0.38 & 0.38 & 0.37 & 0.37 & 0.37 & 0.37 \\
28 & 0 & 3 & 0 & 0 & 0 & 0 & 0 & 0 \\
29 & 0.43 & 0.43 & 0.43 & 0.43 & 0.43 & 0.43 & 0.42 & 0 \\
30 & 3 & 0 & 0 & 0 & 0 & 0 & 0 & 0 \\
\hline
\end{tabular}


Table 17. Components that were held constant in diets used to rear western corn rootworm larvae in the 8-protein mixture experiment.

\begin{tabular}{ll}
\hline \multicolumn{1}{c}{ Components } & Amount \\
\hline 1. Agar & $1.5 \mathrm{~g}$ \\
2. Cellulose & $1.0 \mathrm{~g}$ \\
3. Chlortetracycline $(10 \mathrm{mg} / \mathrm{ml})$ & $6.4 \mathrm{mg}$ \\
4. Cholesterol & $6.0 \mathrm{mg}$ \\
5. Distilled water & $88 \mathrm{ml}$ \\
6. Food coloring & $6.4 \mathrm{mg}$ \\
7. Glucose & $1.0 \mathrm{~g}$ \\
8. Methyl paraben & $0.1 \mathrm{~g}$ \\
9. Potassium hydroxide $(10 \%)$ & $3.5 \mathrm{ml}$ \\
10. Sorbic acid & $6.4 \mathrm{mg}$ \\
11. Streptomycin (12.8 $\mathrm{mg} / \mathrm{ml})$ & $6.4 \mathrm{mg}$ \\
12. Vanderzant vitamin mix & $0.90 \mathrm{~g}$ \\
13. Wesson's salt mix & $0.93 \mathrm{~g}$ \\
14. Wheat germ, ground & $6.0 \mathrm{~g}$ \\
\hline
\end{tabular}


Table 18. Mixture-amount design points to determine the optimum combination of key protein ingredients.

\begin{tabular}{|c|c|c|c|c|}
\hline \multirow{2}{*}{ Diet blend \# } & \multicolumn{3}{|c|}{ Proportion of mixture components } & \multirow{2}{*}{$\begin{array}{c}\text { Total amount of mixture } \\
{[\text { Casein }+ \text { Egg powder }+ \text { Whey }]}\end{array}$} \\
\hline & Casein & Egg powder & Whey & \\
\hline 1 & 0 & 0.5 & 0.5 & 6 \\
\hline 2 & 0.16 & 0.16 & 0.67 & 2.25 \\
\hline 3 & 0.16 & 0.67 & 0.16 & 2.25 \\
\hline 4 & 0.5 & 0 & 0.5 & 6 \\
\hline 5 & 1 & 0 & 0 & 3.5 \\
\hline 6 & 0.5 & 0.5 & 0 & 6 \\
\hline 7 & 1 & 0 & 0 & 6 \\
\hline 8 & 0 & 1 & 0 & 6 \\
\hline 9 & 1 & 0 & 0 & 1 \\
\hline 10 & 0.5 & 0 & 0.5 & 1 \\
\hline 11 & 0.67 & 0.16 & 0.16 & 4.75 \\
\hline 12 & 0.16 & 0.16 & 0.67 & 4.75 \\
\hline 13 & 0.5 & 0.5 & 0 & 1 \\
\hline 14 & 0 & 1 & 0 & 6 \\
\hline 15 & 0 & 1 & 0 & 3.5 \\
\hline 16 & 0 & 0.5 & 0.5 & 1 \\
\hline 17 & 1 & 0 & 0 & 1 \\
\hline 18 & 0 & 0.5 & 0.5 & 3.5 \\
\hline 19 & 0.5 & 0.5 & 0 & 3.5 \\
\hline 20 & 0.5 & 0.5 & 0 & 1 \\
\hline 21 & 0.5 & 0 & 0.5 & 3.5 \\
\hline 22 & 0 & 0 & 1 & 1 \\
\hline 23 & 0 & 0.5 & 0.5 & 1 \\
\hline 24 & 0 & 0 & 1 & 1 \\
\hline 25 & 0.16 & 0.67 & 0.16 & 4.75 \\
\hline 26 & 0 & 1 & 0 & 1 \\
\hline 27 & 0 & 0 & 1 & 3.5 \\
\hline 28 & 0 & 0 & 1 & 6 \\
\hline 29 & 1 & 0 & 0 & 6 \\
\hline 30 & 0 & 1 & 0 & 1 \\
\hline 31 & 0.67 & 0.16 & 0.16 & 2.25 \\
\hline 32 & 0 & 0 & 1 & 6 \\
\hline
\end{tabular}


Table 19. $p$-values, regression coefficients and response surface model fitting diagnostic statistics for NCR larval responses to 8-component diet mixtures.

\begin{tabular}{|c|c|c|c|c|c|c|}
\hline & $\begin{array}{l}\text { Weight } \\
p \text {-values }\end{array}$ & $\begin{array}{l}\text { Regression } \\
\text { coefficients }^{\mathrm{a}}\end{array}$ & $\begin{array}{l}\% \text { Molt } \\
p \text {-values }\end{array}$ & $\begin{array}{l}\text { Regression } \\
\text { coefficients }\end{array}$ & $\begin{array}{l}\% \text { Survival } \\
p \text {-values }\end{array}$ & $\begin{array}{l}\text { Regression } \\
\text { coefficients }\end{array}$ \\
\hline Model & $<0.0001$ & - & $<0.0001$ & - & $<0.0001$ & - \\
\hline Linear mixture & $<0.0001$ & - & $<0.0001$ & - & $<0.0001$ & - \\
\hline A & - & -2.29 & - & -9.28 & - & -0.62 \\
\hline B & - & 2.13 & - & 1.53 & - & 1.12 \\
\hline $\mathrm{C}$ & - & -0.89 & - & -0.53 & - & 1.82 \\
\hline $\mathrm{D}$ & - & 0.22 & - & 9.41 & - & 1.04 \\
\hline $\mathrm{E}$ & - & -0.27 & - & 1.56 & - & 1.04 \\
\hline $\mathrm{F}$ & - & -1.31 & - & -3.41 & - & 0.30 \\
\hline G & - & -21.47 & - & -29.7 & - & -4.25 \\
\hline $\mathrm{H}$ & - & -12.3 & - & -58.09 & - & 1.06 \\
\hline $\mathrm{B}^{2}$ & 0.0354 & -5.97 & - & - & - & - \\
\hline $\mathrm{C}^{2}$ & - & & - & - & $<0.0006$ & -1.92 \\
\hline $\mathrm{D}^{2}$ & 0.0485 & -5.73 & $<0.0001$ & -32.43 & - & - \\
\hline Lack of fit & 0.4138 & & $<0.0001$ & & 0.2778 & \\
\hline Model type & $\begin{array}{l}\text { Quadratic } \\
\text { (reduced) }\end{array}$ & & $\begin{array}{l}\text { Quadratic } \\
\text { (reduced) }\end{array}$ & & $\begin{array}{l}\text { Quadratic } \\
\text { (reduced) }\end{array}$ & \\
\hline Transformation & Base $10 \log$ & & Base $10 \log$ & & N/A & \\
\hline $\mathrm{R}^{2}$ & 0.9633 & & 0.9644 & & 0.9159 & \\
\hline $\mathrm{R}_{\text {adj }}^{2}$ & 0.9378 & & 0.9440 & & 0.8679 & \\
\hline $\mathrm{R}_{\text {pred }}^{2}$ & 0.8296 & & 0.8955 & & 0.7550 & \\
\hline
\end{tabular}

A: agar, B: sucrose, C: wheat germ, D: casein, E: cellulose, F: corn root powder, G: wheat germ oil, F: linseed oil, ${ }^{\text {a }}$ Expressed in coded forms. Mixture component coding is U_Pseudo (Cornell 2002). 
Table 20. $p$-values, regression coefficients and response surface model fitting diagnostic statistics for WCR larval responses to 8-protein diet mixtures.

\begin{tabular}{|c|c|c|c|c|c|c|}
\hline & $\begin{array}{l}\text { Weight } \\
p \text {-values }\end{array}$ & $\begin{array}{l}\text { Regression } \\
\text { coefficients }^{\mathrm{a}}\end{array}$ & $\begin{array}{l}\% \text { Molt } \\
p \text {-values }\end{array}$ & $\begin{array}{l}\text { Regression } \\
\text { coefficients }\end{array}$ & $\begin{array}{l}\% \text { Survival } \\
p \text {-values }\end{array}$ & $\begin{array}{l}\text { Regression } \\
\text { coefficients }\end{array}$ \\
\hline Model & $<0.0001$ & - & $<0.0001$ & - & $<0.0001$ & - \\
\hline Linear mixture & $<0.0001$ & - & $<0.0001$ & - & $<0.0001$ & - \\
\hline A & - & -1.69 & - & -0.02 & - & 0.24 \\
\hline B & - & -1.40 & - & 0.5 & - & 0.90 \\
\hline $\mathrm{C}$ & - & -0.79 & - & 0.91 & - & 0.96 \\
\hline D & - & -1.49 & - & 1.78 & - & 1.86 \\
\hline $\mathrm{E}$ & - & -0.34 & - & 1.78 & - & 1.00 \\
\hline $\mathrm{F}$ & - & 0.12 & - & -0.11 & - & 0.20 \\
\hline G & - & -1.40 & - & 0.58 & - & 0.72 \\
\hline $\mathrm{H}$ & - & 0.88 & - & 0.94 & - & 0.99 \\
\hline$A \times D$ & 0.0029 & 5.95 & - & - & - & - \\
\hline $\mathrm{A} \times \mathrm{F}$ & $<0.0001$ & -17.27 & - & - & - & - \\
\hline $\mathrm{A} \times \mathrm{H}$ & 0.0044 & 5.63 & - & - & - & - \\
\hline $\mathrm{D}^{2}$ & - & - & 0.0084 & -1.46 & $<0.0001$ & -1.15 \\
\hline$E^{2}$ & 0.0198 & -0.73 & 0.0612 & -0.99 & - & - \\
\hline $\mathrm{F}^{2}$ & 0.0021 & -1.61 & - & - & - & - \\
\hline Lack of fit & 0.0649 & & 0.0067 & & 0.0393 & \\
\hline Model type & $\begin{array}{l}\text { Quadratic } \\
\text { (reduced) }\end{array}$ & & $\begin{array}{l}\text { Quadratic } \\
\text { (reduced) }\end{array}$ & & $\begin{array}{l}\text { Quadratic } \\
\text { (reduced) }\end{array}$ & \\
\hline Transformation & N/A & & Square root & & N/A & \\
\hline $\mathrm{R}^{2}$ & 0.9617 & & 0.8805 & & 0.9277 & \\
\hline $\mathrm{R}_{\text {adj }}^{2}$ & 0.9346 & & 0.8267 & & 0.9002 & \\
\hline $\mathrm{R}_{\text {pred }}^{2}$ & 0.7343 & & 0.6317 & & 0.6040 & \\
\hline
\end{tabular}

A: corn gluten meal, B: cottonseed meal, C: casein, D: plant protein, E: whey protein, F: perfect amino, G: yeast extract, $\mathrm{H}$ : egg powder, ${ }^{\mathrm{a}}$ Expressed in coded forms. Mixture component coding is L_Pseudo (Cornell 2002). 
Table 21. $p$-values, regression coefficients and response surface model fitting diagnostic statistics for WCR larval responses to 3-protein mixture-amount experiment.

\begin{tabular}{|c|c|c|c|c|c|c|}
\hline & $\begin{array}{l}\text { Weight } \\
p \text {-values }\end{array}$ & $\begin{array}{l}\text { Regression } \\
\text { coefficients }\end{array}$ & $\begin{array}{l}\% \text { Molt } \\
p \text {-values }\end{array}$ & $\begin{array}{l}\text { Regression } \\
\text { coefficients }\end{array}$ & $\begin{array}{l}\% \text { Survival } \\
p \text {-values }\end{array}$ & $\begin{array}{l}\text { Regression } \\
\text { coefficients }\end{array}$ \\
\hline Model & $<0.0001$ & - & $<0.0001$ & - & $<0.0001$ & - \\
\hline Linear mixture & $<0.0122$ & - & $<0.0207$ & - & $<0.0001$ & - \\
\hline A & - & 0.21 & - & 0.79 & - & 0.96 \\
\hline B & - & 0.13 & - & 0.71 & - & 0.98 \\
\hline $\mathrm{C}$ & - & 0.14 & - & 0.68 & - & 0.87 \\
\hline $\mathrm{A} \times \mathrm{B}$ & - & - & 0.0262 & 0.35 & - & - \\
\hline $\mathrm{A} \times \mathrm{C}$ & 0.0028 & 0.39 & 0.0001 & 0.75 & 0.0387 & -0.12 \\
\hline $\mathrm{B} \times \mathrm{C}$ & - & - & 0.0203 & 0.36 & 0.0318 & 0.11 \\
\hline A $\times$ Conc $^{\mathrm{b}}$ & $<0.0001$ & 0.12 & 0.0004 & 0.14 & - & - \\
\hline B $\times$ Conc. & 0.0934 & -0.04 & - & - & - & - \\
\hline CxConc. & - & - & $<0.0001$ & 0.21 & 0.0030 & 0.03 \\
\hline Cx Conc. $^{2}$ & - & - & - & - & 0.0093 & 0.05 \\
\hline $\mathrm{A} \times \mathrm{C} \times$ Conc. & - & - & 0.0025 & -0.67 & - & - \\
\hline Lack of fit & 0.0368 & & 0.1075 & & 0.6601 & \\
\hline Model type & $\begin{array}{l}\text { Quadratic } \\
\text { mixture } \\
\text { (reduced) } x \\
\text { linear amount }\end{array}$ & & $\begin{array}{l}\text { Quadratic } \\
\text { mixture } \\
\text { (reduced) } \mathrm{x} \\
\text { linear amount }\end{array}$ & & $\begin{array}{l}\text { Quadratic } \\
\text { mixture } \\
\text { (reduced) } \mathrm{x} \\
\text { quadratic } \\
\text { amount } \\
\text { (reduced) }\end{array}$ & \\
\hline Transformation & N/A & & N/A & & N/A & \\
\hline $\mathrm{R}^{2}$ & 0.6714 & & 0.8369 & & 0.6964 & \\
\hline $\mathrm{R}_{\text {adj }}^{2}$ & 0.6057 & & 0.7776 & & 0.6173 & \\
\hline $\mathrm{R}_{\text {pred }}^{2}$ & 0.4407 & & 0.6490 & & 0.4138 & \\
\hline
\end{tabular}

A: egg powder, B: whey protein, C: casein. ${ }^{a}$ Mixture component coding is L_Pseudo (Cornell 2002); ${ }^{\mathrm{b}}$ Concentration 
Table 22. An artificial diet for NCR larvae.

\begin{tabular}{lcc}
\hline Ingredients & WCRMO-1 (Huynh et al. 2017) & NCRMO-1 \\
\hline Egg powder & - & + \\
Glucose & - & + \\
Casein & + & + \\
Wheat germ (raw, ground) & + & + \\
Cellulose & + & + \\
Agar & + & + \\
Corn root powder & + & - \\
Sucrose & + & - \\
Linseed oil, raw & + & - \\
Wheat germ oil & + & - \\
Cholesterol & + & + \\
Wesson's salt mix & + & + \\
Vanderzant Vitamin mix & + & + \\
Methyl paraben & + & + \\
Sorbic acid & + & + \\
Potassium hydroxide $(10 \%)$ & + & + \\
Streptomycin $(12.8 \mathrm{mg} / \mathrm{ml})$ & + & + \\
Chlortetracycline $(10.0 \mathrm{mg} / \mathrm{ml})$ & + & + \\
Distilled water & + & + \\
Green food coloring & + & + \\
\hline & & +
\end{tabular}



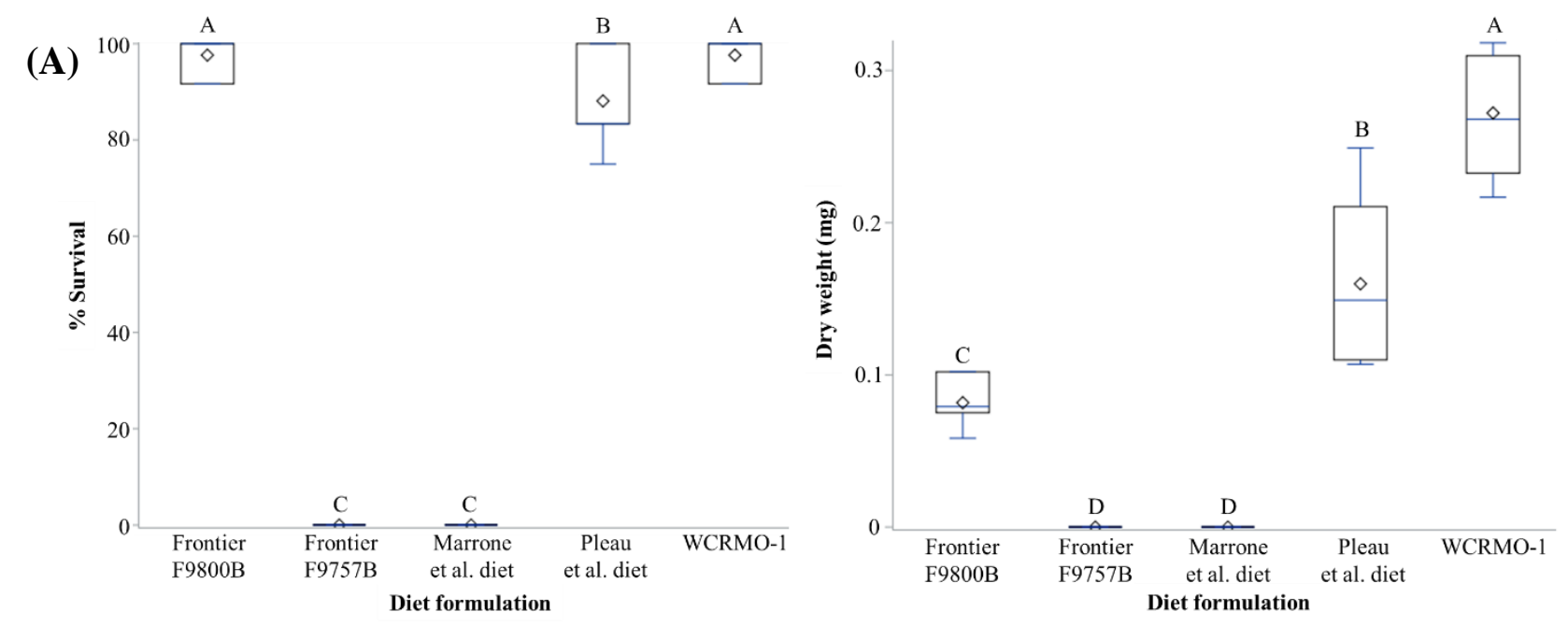

(B)

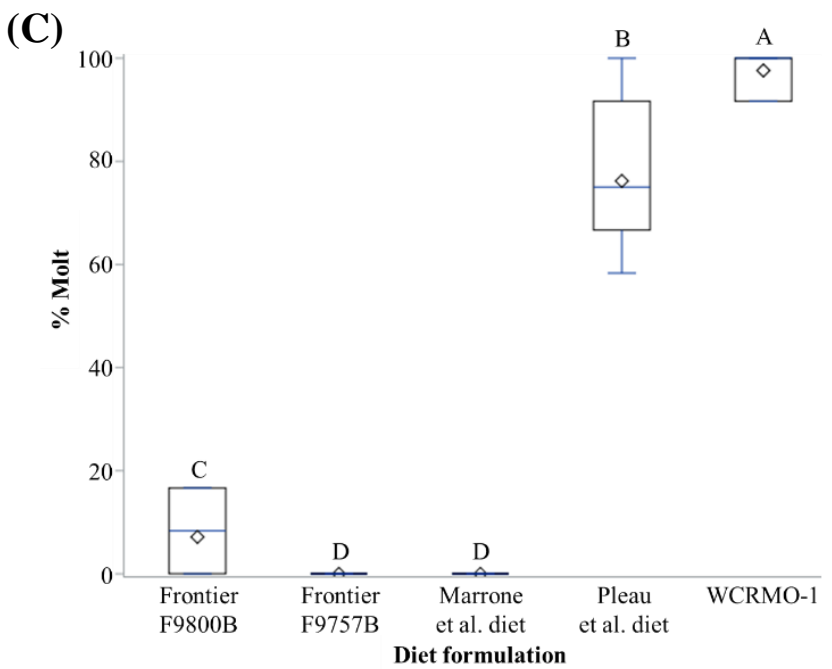

Figure 16. Percentage survival (A), average dry weight (B), percentage molting to $2^{\text {nd }}$ instar (C), of northern corn rootworm larvae fed different diet formulations for 10 days. Frontier SCR diets (F9800B \& F9757B, Frontier Newark, DE), the Marrone et al. diet (Marrone et al. 1985), the Pleau et al. diet (Pleau et al. 2002), the WCRMO-1 diet (Huynh et al. 2017). Bars with different letters are significantly different $(\mathrm{p}<0.0001)$. 

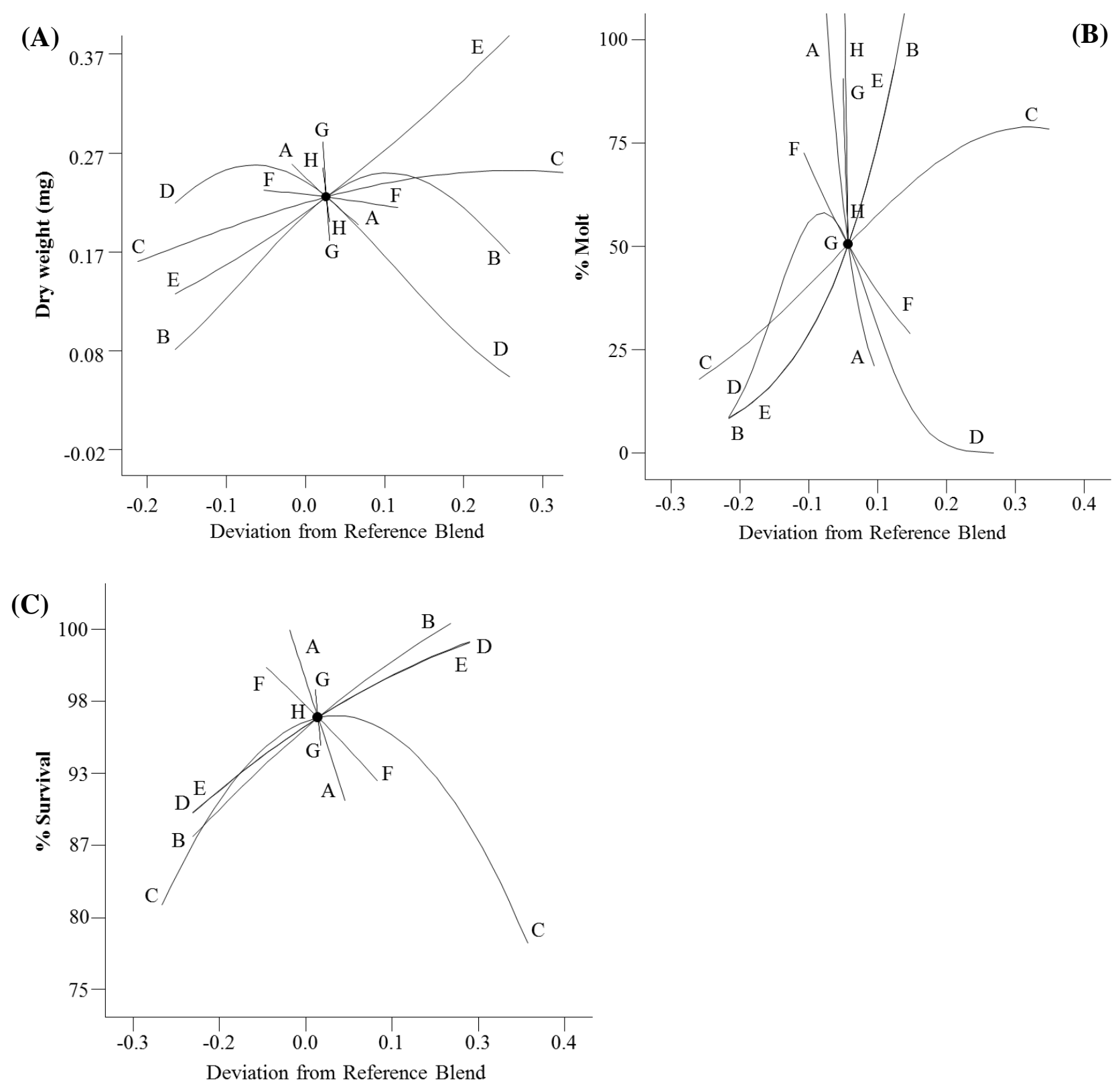

Figure 17. Cox plot of larval responses to deviations from a reference blend diet in the proportions of ingredients in an 8-component mixture experiment. (A) weight, (B) molting, (C) survival. Reference blend proportions: agar $=0.046$, sucrose $=0.203$, wheat germ $=0.253$, casein $=0.203$, cellulose $=0.203$, corn root: 0.075 , wheat germ oil $=0.009$, linseed oil $=0.009$. A: agar, B: sucrose, C: wheat germ, D: casein, E: cellulose, F: corn root, G: wheat germ oil, H: linseed oil. 

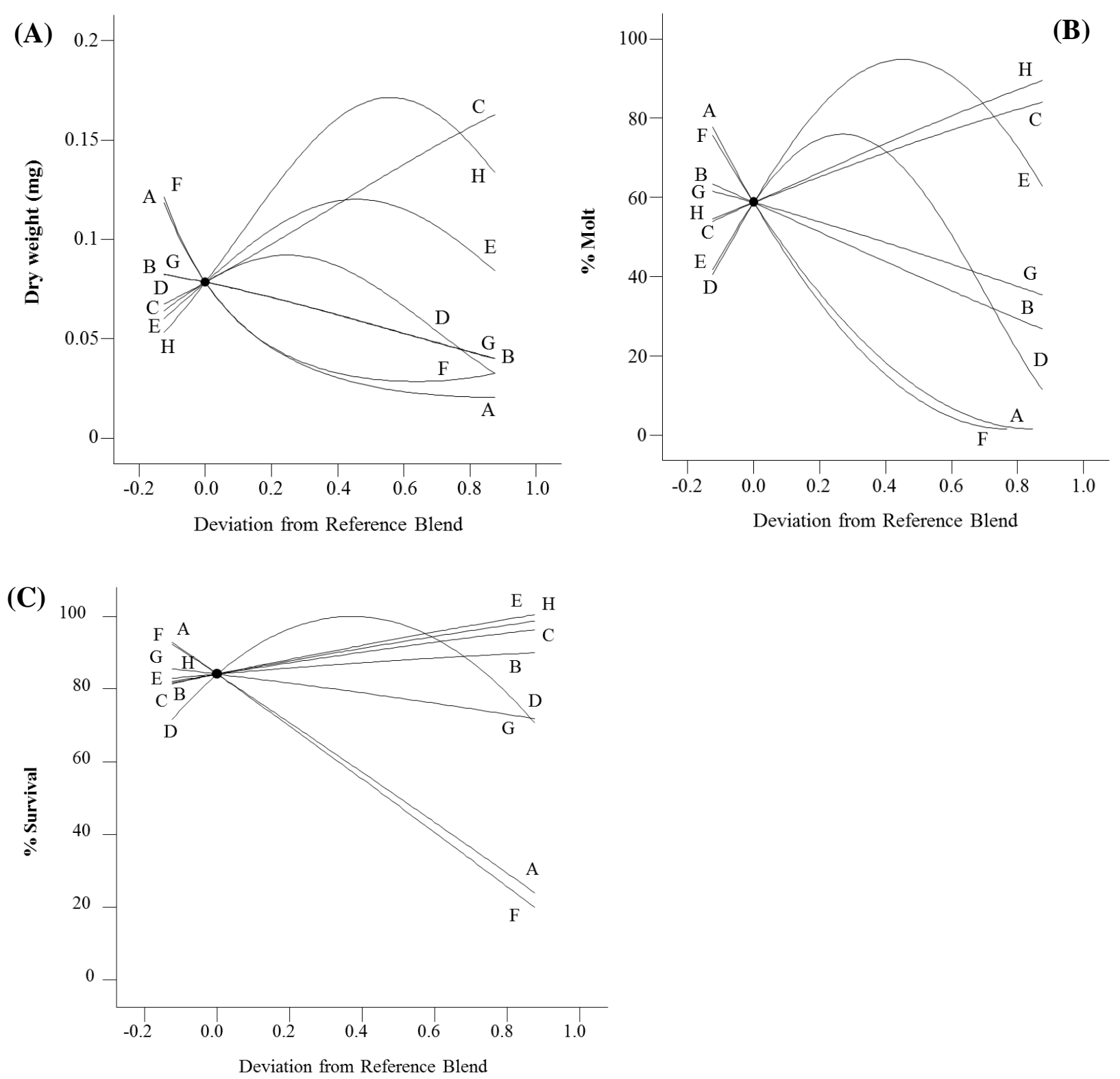

Figure 18. Cox plot of larval responses to deviations from a reference blend diet in the proportions of ingredients in an 8-protein screening experiment. (A) weight, (B) molt, (C) survival. Reference blend proportions: corn gluten meal $=$ cottonseed meal $=$ casein $=$ plant protein $=$ whey protein $=$ perfect amino $=$ yeast extract $=$ egg powder $=0.375$. A: corn gluten meal, B: cottonseed meal, C: casein, D: plant protein, E: whey protein, F: perfect amino, G: yeast extract, H: egg powder. 

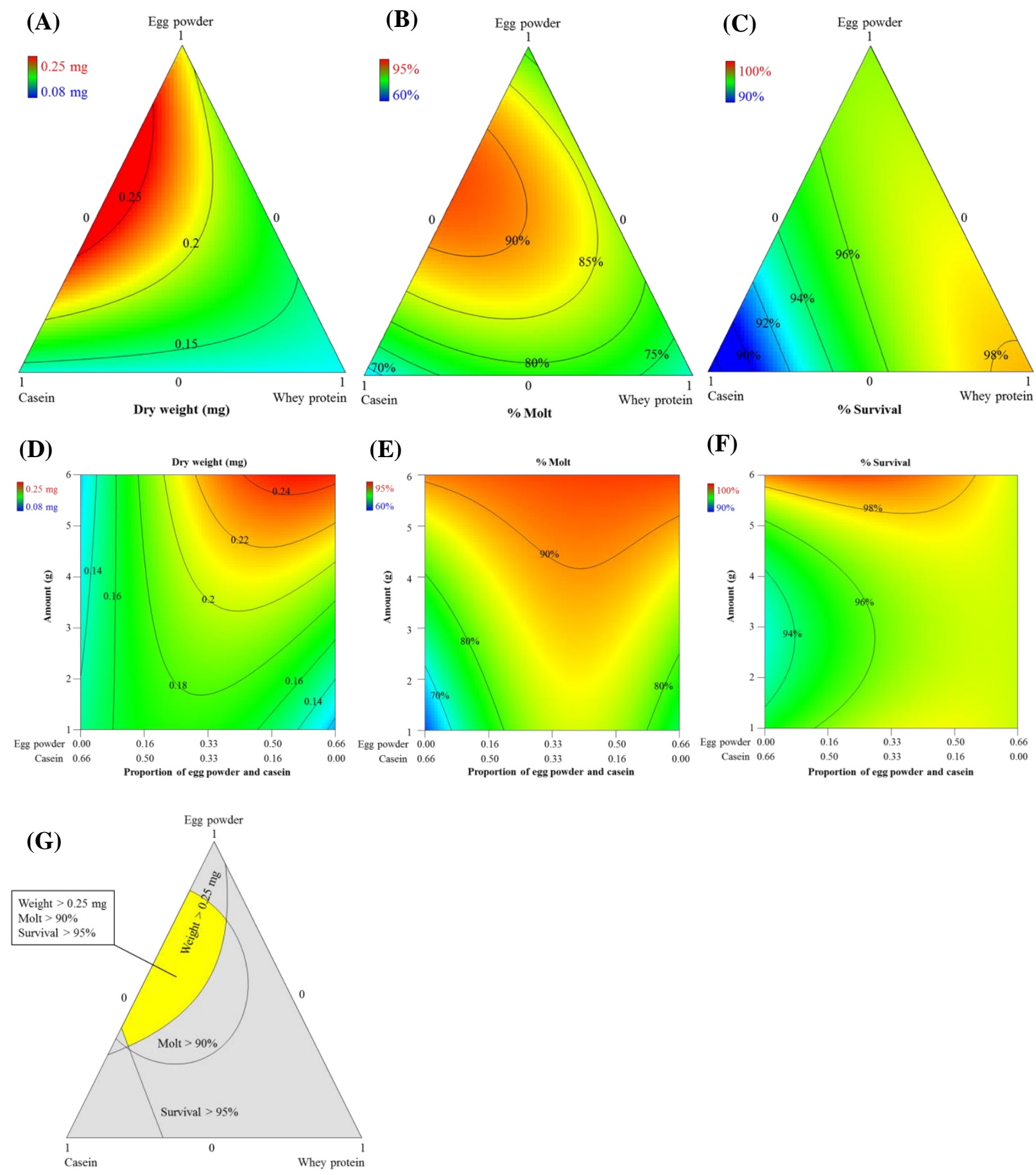

Figure 19. Contour plots of larval responses to a mixture-amount design of casein:egg powder:whey protein at 10 days post infestation. (A) weight, (B) survival, and (C) molting. (DF) interaction of egg powder, casein and concentration on (D) weight, (E) survival, and (F) molting; proportions of whey protein $=0.34$. Color bars display the magnitude of the measured response. (G) Overlaid optimization plot of larval responses; yellow area represents the optimum region for weight, molting, and survival that are obtained when three ingredients (i.e., egg powder, casein, whey protein) are used. 

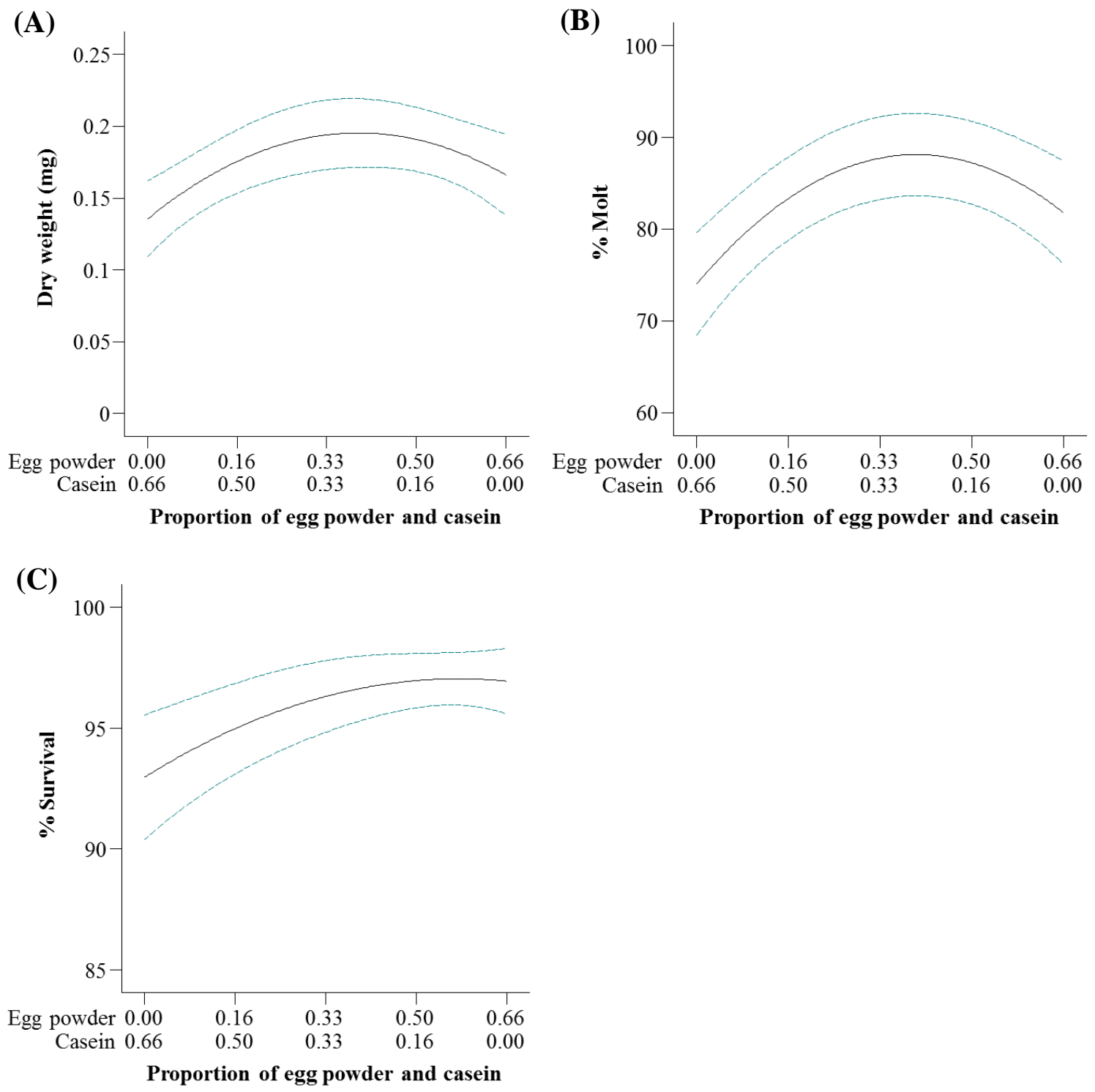

Figure 20. Nonlinear blending effects of casein $\times$ egg powder from the mixture-amount experiment. (A) weight, (B) molting, and (C) survival. Dotted lines indicate $95 \%$ confidence interval bands. Proportion of whey protein $=0.34$. Total amount of mixture $=3$ grams. 


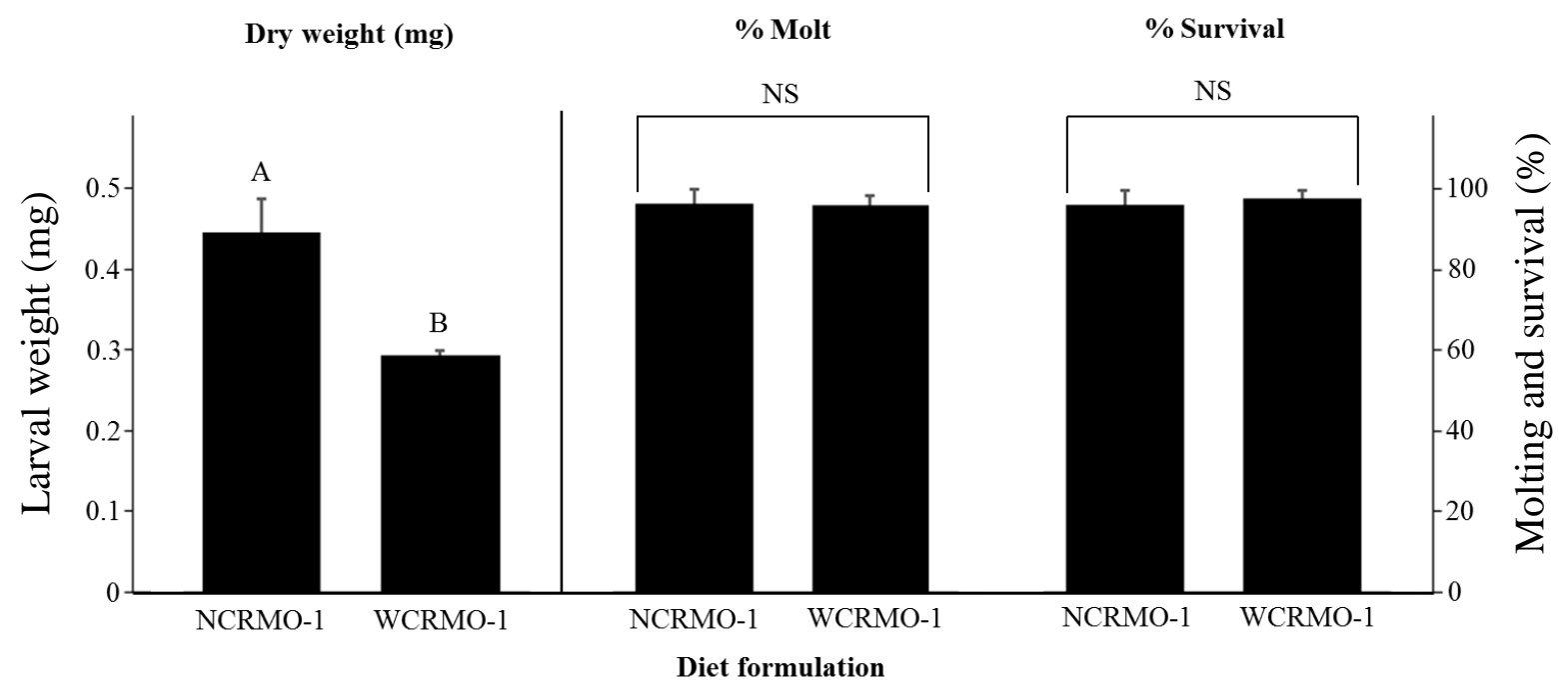

Figure 21. Larval dry weight, percent successful completion of molt, and survival for northern corn rootworm larvae reared on NCRMO-1 and WCRMO-1 diets for 10 days. Means within bars followed by different letters are significantly different $(\mathrm{p}<0.05)$. Mean \pm SEM. 


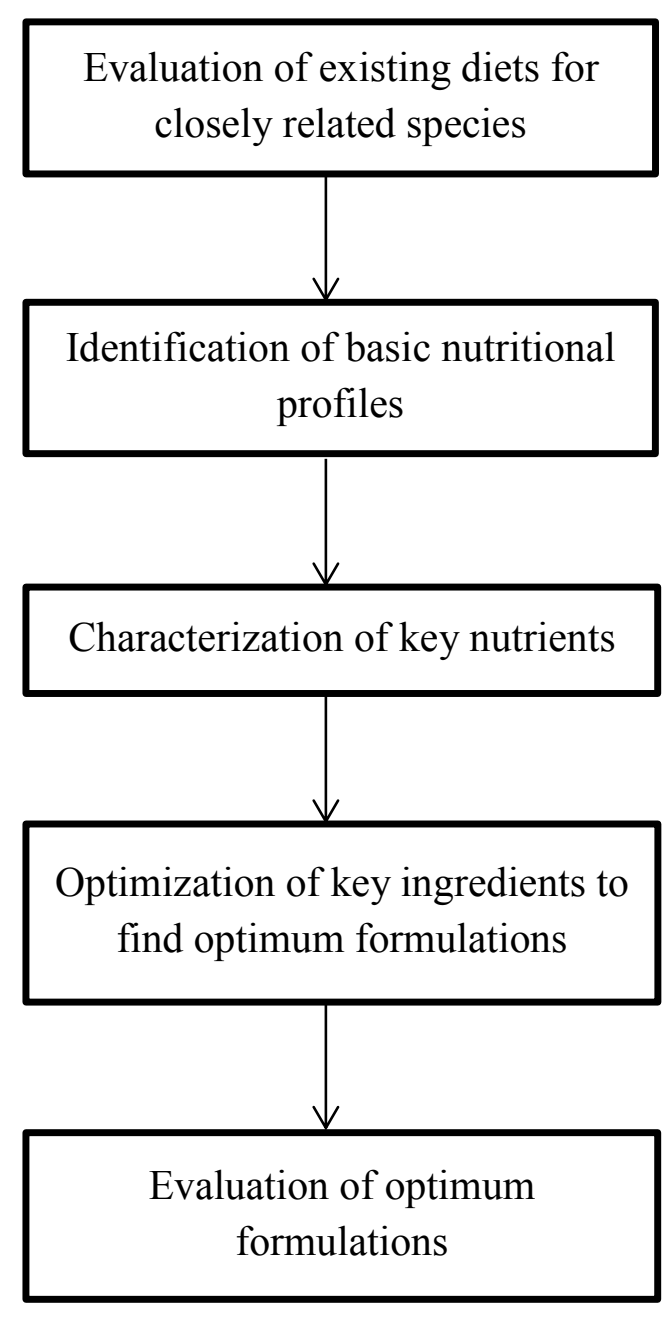

Figure 22. Flow chart for diet development algorithm 


\section{References}

Allen, D. M. 1971. Mean square error of prediction as a criterion for selecting variables. Technometrics 13: 469-475.

Anderson, M. J., and P. J. Whitcomb. 2004. RSM simplified: optimizing processes using response surface methods for design of experiments, CRC press.

Anderson, M. J., and P. J. Whitcomb. 2007. Using graphical diagnostics to deal with bad data. Qual. Eng. 19: 111-118.

Beck, S. D., and J. F. Stauffer. 1950. An aseptic method for rearing European corn borer larvae. J. Econ. Entomol. 43: 4-6.

Bernklau, E. J., and L. B. Bjostad. 2008. Identification of feeding stimulants in corn roots for western corn rootworm (Coleoptera: Chrysomelidae) larvae. J. Econ. Entomol. 101: 341351.

Bernklau, E. J., B. E. Hibbard, A. P. Norton, and L. B. Bjostad. 2016. Methyl anthranilate as a repellent for western corn rootworm larvae (Coleoptera: Chrysomelidae). J. Econ. Entomol. 109: 1683-1690.

Bernklau, E. J., B. E. Hibbard, D. L. Dick, C. D. Rithner, and L. B. Bjostad. 2015. Monogalactosyldiacylglycerols as host recognition cues for western corn rootworm larvae (Coleoptera: Chrysomelidae). J. Econ. Entomol. 108: 539-548.

Cohen, A. C. 2015. Insect diets: science and technology, 2nd ed., Taylor \& Francis Group.

Cornell, J. A. 2002. Experiments with mixtures: designs, models, and the analysis of mixture data, 3rd ed. John Wiley \& Sons, Inc., New York, USA.

Czitrom, V. 1988. Mixture experiments with process variables: D-optimal orthogonal experimental designs. Commun. Stat. Theory Methods 17: 105-121.

Derringer, G. 1980. Simultaneous optimization of several response variables. J. Qual. Technol. 12: 214-219.

Gassmann, A. J., J. L. Petzold-Maxwell, R. S. Keweshan, and M. W. Dunbar. 2011. Fieldevolved resistance to Bt maize by western corn rootworm. PloS one 6: e22629. 
Goos, P., B. Jones, and U. Syafitri. 2016. I-Optimal design of mixture experiments. J. Am. Stat. Assoc. 111: 899-911.

Hamilton, E. W. 1965. Aldrin resistance in corn rootworm beetles. J. Econ. Entomol. 58: 296300.

Huynh, M. P., L. N. Meihls, B. E. Hibbard, S. L. Lapointe, R. P. Niedz, D. C. Ludwick, and T. A. Coudron. 2017. Diet improvement for western corn rootworm (Coleoptera: Chrysomelidae) larvae. PloS one 12: e0187997.

Kahler, A., A. Olness, G. Sutter, C. Dybing, and O. Devine. 1985. Root damage by western corn rootworm and nutrient content in maize. Agron. J. 77: 769-774.

Krysan, J., D. Foster, T. Branson, K. Ostlie, and W. Cranshaw. 1986. Two years before the hatch: rootworms adapt to crop rotation. Bull. ESA 32: 250-253.

Kurtz, B., P. Karlovsky, and S. Vidal. 2010. Interaction between western corn rootworm (Coleoptera: Chrysomelidae) larvae and root-infecting Fusarium verticillioides. Environ. Entomol. 39: 1532-1538.

Laake, P. 1975. On the optimal allocation of observations in experiments with mixtures. Scand. J. Stat. 2: 153-157.

Lapointe, S. L., T. J. Evens, and R. P. Niedz. 2008. Insect diets as mixtures: Optimization for a polyphagous weevil. J. Ins. Physiol. 54: 1157-1167.

Lapointe, S. L., R. P. Niedz, and T. J. Evens. 2010a. An artificial diet for Diaprepes abbreviatus (Coleoptera: Curculionidae) optimized for larval survival. Fla. Entomol. 93: 56-62.

Lapointe, S. L., T. J. Evens, R. P. Niedz, and D. G. Hall. 2010b. Artificial diet optimized to produce normative adults of Diaprepes abbreviatus (Coleoptera: Curculionidae). Environ. Entomol. 39: 670-677.

Lee, K. P., S. J. Simpson, F. J. Clissold, R. Brooks, J. W. Ballard, P. W. Taylor, N. Soran, and D. Raubenheimer. 2008. Lifespan and reproduction in Drosophila: New insights from nutritional geometry. Proc. Nat. Acad. Sci. 105: 2498-2503.

Lindig, O., and O. Malone. 1973. Oviposition of boll weevils fed diets containing germinated cottonseed puree or cottonseed meats puree. J. Econ. Entomol. 66: 566-567. 
Ludwick, D. C., L. N. Meihls, K. R. Ostlie, B. D. Potter, L. French, and B. E. Hibbard. 2017. Minnesota field population of western corn rootworm (Coleoptera: Chrysomelidae) shows incomplete resistance to Cry34Ab1/Cry35Ab1 and Cry3Bb1. J. Appl. Entomol. 141: $28-40$.

Ludwick, D. C., L. N. Meihls, M. P. Huynh, A. E. Pereira, B. W. French, T. A. Coudron, and B. E. Hibbard. 2018. A new artificial diet for western corn rootworm larvae is compatible with and detects resistance to all current Bt toxins. Sci. Rep. 8: 5379.

Marrone, P. G., F. D. Ferri, T. R. Mosley, and L. J. Meinke. 1985. Improvements in laboratory rearing of the southern corn rootworm, Diabrotica undecimpuncta howardi Barber (Coleoptera: Chrysomelidae), on an artificial diet and corn. J. Econ. Entomol. 78: 290293.

Mitchell, P. D. 2011. Costs and benefits of controlling pest Diabrotica in maize in the United States. In, 24th IWGO Conference and 3rd International Conference of Diabrotica Genetics, IWGO, Freiburg, Germany.

Myers, R. H., and D. C. Montgomery. 2002. Response surface methodology: process and product optimization using designed experiments, 2nd ed., Willey, New York, NY, USA.

Myers, R. H., D. C. Montgomery, and C. M. Anderson-Cook. 2016. Response surface methodology: process and product optimization using designed experiments, 4th ed., John Wiley \& Sons, New York, NY, USA.

Niedz, R. P., and T. J. Evens. 2016. Design of experiments (DOE)—history, concepts, and relevance to in vitro culture. In Vitro Cell. Dev. Biol. - Plant 52: 547-562.

Oyediran, I. O., B. E. Hibbard, T. L. Clark, and B. W. French. 2004. Selected grassy weeds as alternate hosts of northern corn rootworm (Coleoptera: Chrysomelidae). Environ. Entomol. 33: 1497-1504.

Oyediran, I. O., B. W. French, T. L. Clark, K. E. Dashiell, and B. E. Hibbard. 2008. Prairie grasses as hosts of the northern corn rootworm (Coleoptera: Chrysomelidae). Environ. Entomol. 37: 247-254. 
Oyediran, I. O., P. Matthews, N. Palekar, W. French, J. Conville, and T. Burd. 2016. Susceptibility of northern corn rootworm Diabrotica barberi (Coleoptera: Chrysomelidae) to mCry3A and eCry3.1Ab Bacillus thuringiensis proteins. Insect Sci. 23: 913-917.

Palmer, L., and T. Kommedahl. 1969. Root-infecting Fusarium species in relation to rootworm infestations in corn. Phytopathology 59:1613-1617.

Pereira, A. E., N. P. Carneiro, and B. D. Siegfried. 2016. Comparative susceptibility of southern and western corn rootworm adults and larvae to vATPase-A and Snf7 dsRNAs. J. RNAi and Gene Silencing 12: 528-535.

Pleau, M. J., J. E. Huesing, G. P. Head, and D. J. Feir. 2002. Development of an artificial diet for the western corn rootworm. Entomol. Exp. Appl. 105: 1-11.

Raubenheimer, D. 2011. Toward a quantitative nutritional ecology: the right-angled mixture triangle. Ecol. Monogr. 81: 407-427.

Rose, R. I., and J. M. McCabe. 1973. Laboratory rearing techniques for the southern corn rootworm. J. Econ. Entomol. 66: 398-400.

SAS. 2013. SAS version 9.4, SAS Institute, Cary, N.C, USA.

Scheffé, H. 1958. Experiments with mixtures. Royal Stat. Soc., Series B (Methodological) 20: 344-360.

Smith, W. F. 2005. Experimental design for formulation. ASA-SIAM series on statistics and applied probability. SIAM, Philadelphia, PA, USA.

Spike, B. P., and J. J. Tollefson. 1988. Western corn rootworm (Coleoptera: Chrysomelidae) larval survival and damage potential to corn subjected to nitrogen and plant density treatments. J. Econ. Entomol. 81: 1450-1455.

Spike, B. P., and J. J. Tollefson. 1989. Relationship of plant phenology to corn yield loss resulting from western corn rootworm (Coleoptera: Chrysomelidae) larval injury, nitrogen deficiency, and high plant density. J. Econ. Entomol. 82: 226-231. 
Spike, B. P., and J. J. Tollefson. 1991. Yield response of corn subjected to western corn root worm (Coleoptera: Chrysomelidae) infestation and lodging. J. Econ. Entomol. 84: 15851590.

Sutter, G. R., J. L. Krysan, and P. L. Guss. 1971. Rearing the southern corn rootworm on artificial diet. J. Econ. Entomol. 64: 65-67.

Tan, X. L., S. Wang, and F. Zhang. 2013. Optimization an optimal artificial diet for the predatory bug Orius sauteri (Hemiptera: Anthocoridae). PloS one 8: e61129.

Vanderzant, E. S., and R. Reiser. 1956a. Aseptic rearing of the pink bollworm on synthetic media. J. Econ. Entomol. 49: 7-10.

Vanderzant, E. S., and R. Reiser. 1956b. Studies of the nutrition of the pink bollworm using purified casein media. J. Econ. Entomol. 49: 454-458.

Vanderzant, E. S., R. Reiser, and E. Ivy. 1956. Methods for the mass rearing of the pink bollworm. J. Econ. Entomol. 49: 559-560.

Zhao, J. Z., M. A. Oneal, N. M. Richtman, S. D. Thompson, M. C. Cowart, M. E. Nelson, Z. Pan, A. P. Alves, and T. Yamamoto. 2016. mCry3A-selected western corn rootworm (Coleoptera: Chrysomelidae) colony exhibits high resistance and has reduced binding of mCry3A to midgut tissue. J. Econ. Entomol. 109: 1369-1377.

Zukoff, S. N., K. R. Ostlie, B. Potter, L. N. Meihls, A. L. Zukoff, L. French, M. R. Ellersieck, B. Wade French, and B. E. Hibbard. 2016. Multiple assays indicate varying levels of cross resistance in Cry3Bb1-selected field populations of the western corn rootworm to mCry3A, eCry3.1Ab, and Cry34/35Ab1. J. Econ. Entomol. 109: 1387-1398. 


\section{VITA}

Man P. Huynh was born in Can Tho city, Vietnam. He graduated from the Can Tho University in Can Tho city, Vietnam in 2008 with a bachelor's degree in Agronomy. He conducted his undergraduate research in Dr. Vang Le lab in the years of 2006-2008. His bachelor's thesis was entitled "Natural enemies of common cutworm (Lepidoptera: Noctuidae; Spodoptera litura Fabricius) and mass rearing of the larval parasitoid (Hymenoptera:

Braconidae; Microplitis manilae Ashmead). He continued his M.S. program in Dr. Vang Le lab in terms of 2008-2010 and his master's thesis was entitled "Systematics and management for bagworms (Lepidoptera: Psychidae) in nipa palm (Nypa fruticans Wurmb) in Mekong Delta and chemical ecology of Plagiophelps sp.”. Man Huynh received a master's degree in Plant Protection at the Can Tho University in 2010. After graduation in 2008, he started to work as a research specialist and teaching assistant in the Department of Plant Protection at the Can Tho University and, four years later (2012), he became a faculty member at the Can Tho University.

As a faculty member, his responsibilities include research, teaching and extension. He started his Ph.D. in 2014 at the University of Missouri with Drs. Deborah Finke and Thomas Coudron. 